\title{
Information retrieval by graphically browsing meta- information
}

Citation for published version (APA):

Wiesman, F. J. (1998). Information retrieval by graphically browsing meta-information. [Doctoral Thesis, Maastricht University]. Phidippides. https://doi.org/10.26481/dis.19980507fw

Document status and date:

Published: 01/01/1998

DOI:

10.26481/dis.19980507fw

Document Version:

Publisher's PDF, also known as Version of record

\section{Please check the document version of this publication:}

- A submitted manuscript is the version of the article upon submission and before peer-review. There can be important differences between the submitted version and the official published version of record.

People interested in the research are advised to contact the author for the final version of the publication, or visit the DOI to the publisher's website.

- The final author version and the galley proof are versions of the publication after peer review.

- The final published version features the final layout of the paper including the volume, issue and page numbers.

Link to publication

\footnotetext{
General rights rights.

- You may freely distribute the URL identifying the publication in the public portal. please follow below link for the End User Agreement:

www.umlib.nl/taverne-license

Take down policy

If you believe that this document breaches copyright please contact us at:

repository@maastrichtuniversity.nl

providing details and we will investigate your claim.
}

Copyright and moral rights for the publications made accessible in the public portal are retained by the authors and/or other copyright owners and it is a condition of accessing publications that users recognise and abide by the legal requirements associated with these

- Users may download and print one copy of any publication from the public portal for the purpose of private study or research.

- You may not further distribute the material or use it for any profit-making activity or commercial gain

If the publication is distributed under the terms of Article $25 \mathrm{fa}$ of the Dutch Copyright Act, indicated by the "Taverne" license above, 


\section{Information Retrieval}

by

Graphically Browsing Meta-Information 



\section{Information Retrieval by \\ Graphically Browsing Meta-Information}

\section{PROEFSCHRIFT}

ter verkrijging van de graad van doctor aan de Universiteit Maastricht, op gezag van de Rector Magnificus, Prof. dr. A.C. Nieuwenhuijzen Kruseman, volgens het besluit van het College van Decanen, in het openbaar te verdedigen

op donderdag 7 mei 1998, om 14.00 uur

door

Florimond Jan Wiesman 
Promotores: Prof. dr. ir. A. Hasman

Prof. dr. H.J. van den Herik

Prof. dr. ir. J.L.G. Dietz (Technische Universiteit Delft)

Leden van de beoordelingscommissie:

Prof. dr. C.P.M. van der Vleuten (voorzitter)

Prof. dr. J.W. Arends

Prof. dr. ir. K.L. Boon

Prof. dr. P.T.W. Hudson

Prof. dr. H. Koppelaar (Technische Universiteit Delft)

的 Dissertation Series No. 98-2

ISBN 90-801577-3-2

Uitgeverij Phidippides, Cadier en Keer

(C)1998 Floris Wiesman

Cover design: Giselle de Oliveira 


\section{Contents}

$\begin{array}{ll}\text { Preface } & \text { ix }\end{array}$

1 Introduction 1

1.1 Finding Information . . . . . . . . . . . . . . . . . 2

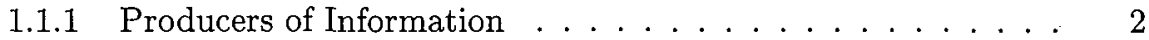

1.1.2 Users of Information . . . . . . . . . . . . . . . . 2

1.1 .3 Students . . . . . . . . . . . . . . . 3

1.2 Finding the Right Information . . . . . . . . . . . . 3

1.2.1 The Information Need . . . . . . . . . . . . . . . . . 4

1.2.2 Communication between the IR System and its Users . . . . 4

1.2.3 Communication between Humans . . . . . . . . . . . 5

1.2 .4 Knowledge . . . . . . . . . . . . . . . 5

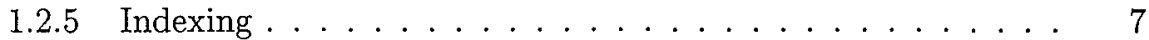

1.2 .6 Reliability . . . . . . . . . . . . . 7

1.3 Querying versus Browsing . . . . . . . . . . . . 7

1.3.1 Cove and Walsh' Browsing Types . . . . . . . . . 8

1.3.2 Chang and Rice's Browsing Dimensions ... . . . . . . . 8

1.3.3 Waterworth and Chignell's Information Exploration Classification . . . . . . . . . . . . . . 9

1.3.4 Conclusions on Querying versus Browsing . . . . . . . . 10

1.4 The Archimedes Project . . . . . . . . . . . . . . . . . . 10

1.5 Problem Statement and Hypotheses . . . . . . . . . . . . . 11

1.6 Thesis Outline . . . . . . . . . . . . . . . . . . . . . 13

2 Information Retrieval: An Overview of System Characteristics 15

2.1 Background . . . . . . . . . . . . . . . . 15

2.2 The Information Retrieval Model Characteristic . . . . . . . . . . 17

2.2 .1 The Boolean Model . . . . . . . . . . . . . . . . 17

2.2 .2 The Vector Model . . . . . . . . . . . . . . . . . 18

2.2 .3 The Probabilistic Model . . . . . . . . . . . . . . . . . 21

2.2.4 The Connectionistic Model . . . . . . . . . . . . 22 
2.3 The Twelve Characteristics . . . . . . . . . . . . . . . . . 24

2.3 .1 Search Intermediaries $\ldots \ldots \ldots \ldots \ldots$

2.3.2 Domain Knowledge . . . . . . . . . . . . . . . . . . . 27

2.3.3 Relevance Feedback . . . . . . . . . . . . . . . . . 28

2.3.4 Natural-Language Interface . . . . . . . . . . . . . . . . . 29

2.3.5 Graphical Query Language . . . . . . . . . . . . . . . 29

2.3.6 Conceptual Queries . . . . . . . . . . . . . . . . . 30

2.3 .7 Full-Text IR . . . . . . . . . . . . . . . . . . . . . . . . . . . 31

2.3 .8 Field Searching . . . . . . . . . . . . . . . 31

2.3 .9 Fuzzy Queries . . . . . . . . . . . . . . . . . 32

2.3 .10 Hypertext Integration . . . . . . . . . . . . . . 32

2.3.11 Machine Learning . . . . . . . . . . . . . . . . . 33

2.3 .12 Ranked Output . . . . . . . . . . . . . . . 34

2.4 Information Retrieval and the World Wide Web . . . . . . . . 36

2.4.1 The Word Wide Web . . . . . . . . . . . . . . 36

2.4 .2 Search Engines . . . . . . . . . . . . . . . . 37

2.5 Chapter Conclusions . . . . . . . . . . . . . . . . . . 39

2.5.1 Conclusions on Current IR Systems . . . . . . . . . . . 39

2.5.2 Requirements for the Archimedes IR System . . . . . . . 40

3 Design of the Archimedes Network System 41

3.1 The Information to Be Searched and Accessed . . . . . . . . . . . 42

3.1 .1 Document Types . . . . . . . . . . . . . . . . . . . 42

3.1 .2 Document Properties . . . . . . . . . . . . . . . . 45

3.2 The ANS Architecture . . . . . . . . . . . . . . . . 49

3.2.1 The ANS Approach to Search and Access . . . . . . . . 49

3.2 .2 The Architecture of an ANS Implementation . . . . . . . . 51

3.3 The ANS Information Network . . . . . . . . . . . . 53

3.3 .1 The ANS Factbase (ANS-FB) . . . . . . . . . 54

3.3.2 The ANS Conceptual Schema (ANS-CS) . . . . . . . . . 54

3.3 .3 The ANS Metaschema (ANS-MS) . . . . . . . . 56

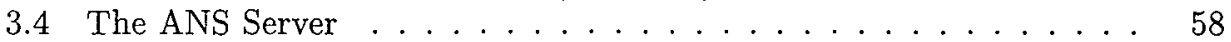

3.5 An Informal Evaluation . . . . . . . . . . . . . . 63

3.6 Chapter Conclusions . . . . . . . . . . . . . . . 63

4 Implementation of the Archimedes Network System 65

4.1 The Archimedes Information Network . . . . . . . . . . . . 65

4.2 Implemented Information Networks . . . . . . . . . . . . . 68

4.2.1 The Medline Information Network . . . . . . . . . . . . 69

4.2.2 The Virtual Hospital Information Network . . . . . . . . 71

4.2.3 The Paleopathology Information Network . . . . . . . . . . 72

4.2.4 The Transnational Income Tax Information Network . . . . . 73

4.3 Document Access Programs . . . . . . . . . . . . . . . . . 73 
4.4 The ANS Browser $\ldots \ldots \ldots \ldots \ldots \ldots \ldots \ldots$

4.4.1 Browsing through the Information Network . . . . . . . 75

4.4.2 Visualization of the Information Network . . . . . . . 78

4.4 .3 Navigation Aids . . . . . . . . . . . . . . . 81

4.4 .4 Combination of Key Terms . . . . . . . . . . . . 83

4.5 Chapter Conclusions . . . . . . . . . . . . . . . . . . 85

5 Protocol for Evaluation $\quad \mathbf{8 7}$

5.1 The Problem of Evaluating an IR System . . . . . . . . . . 87

5.1 .1 Terminology in IR System Evaluation . . . . . . . . . 87

5.1.2 Designs of Evaluation Experiments . . . . . . . . . . 88

5.1.3 Measures for Effectiveness and Efficiency . . . . . . . . 88

5.1 .4 The Document Set . . . . . . . . . . . . . . . . . 91 91

5.1 .5 The Questions .................... . . 91

5.1 .6 The Retrieved Set . . . . . . . . . . . . . . . . . 92

5.1 .7 Relevance . . . . . . . . . . . . . . . . . . 92

5.1 .8 The Users . . . . . . . . . . . . . . . . . . . . . . . . . . 93

5.1.9 Conclusion on IR System Evaluation Literature . . . . . . . 93

5.2 Protocol . . . . . . . . . . . . . . . . . . . . . . 93

5.2 .1 Objective and Features of the Experiment . . . . . . . . 94

5.2 .2 Material . . . . . . . . . . . . . . . . . . . 95

5.2 .3 Operationalization . . . . . . . . . . . . . . . . 96

5.2 .4 Design . . . . . . . . . . . . . . . . . 96

6 Evaluation Results 101

6.1 Comparison of the Relevance Judgements . . . . . . . . . . . . 101

6.2 Comparison of the Systems . . . . . . . . . . . . . . 103

6.2 .1 Method of Analysis . . . . . . . . . . . . . . . 103

6.2 .2 Recall . . . . . . . . . . . . . . . . . . 105

6.2 .3 Precision . . . . . . . . . . . . . . 107

6.2 .4 Rijsbergen's Measure . . . . . . . . . . . . . . . . . . 108

6.2 .5 Time Used . . . . . . . . . . . . . . . . . . . . 109

6.2 .6 User Satisfaction . . . . . . . . . . . . . . . . . . . 111

6.2.7 An Extension of the Experimental Design . . . . . . . . . 111

6.3 Analysis of Searches . . . . . . . . . . . . . . . . 113

6.3.1 Two Characteristic Cases . . . . . . . . . . . . . 113

6.3.2 Differences in Retrieval Frequencies . . . . . . . . . 115

6.3.3 Documents Found Only Once . . . . . . . . . . . . . . 115

6.3.4 Found with the Extended ANS Only . . . . . . . . . . 118

6.3.5 Correlations with User Satisfaction . . . . . . . . . . . . 119

6.4 Search Behaviour in ANS . . . . . . . . . . . . . . . . . . . 119

6.5 Search Behaviour in WinSpirs . . . . . . . . . . . . . . . 121

6.6 Interviews . . . . . . . . . . . . . . . . . . . . 123 
6.6.1 Information Retrieval Experience . . . . . . . . . . . . 123

6.6.2 Missing Features and Other Complaints . . . . . . . . . . . 124

6.6 .3 Link Type Usage . . . . . . . . . . . . . . . . . . . 125

6.6 .4 Filters . . . . . . . . . . . . . . . . . 125

6.6.5 Usage of the WinSpirs Thesaurus . . . . . . . . . . . 126

6.6.6 Difficulty with English in the Search Questions . . . . . . . 126

6.6.7 Clarity of the Search Questions . . . . . . . . . . . . 127

6.6 .8 Learning Effect . . . . . . . . . . . . . . . . . . . 127

6.7 Conclusions on the Experiment $\ldots \ldots \ldots \ldots \ldots \ldots$

7 Discussion and Conclusions 131

7.1 The Information Network and the Browser . . . . . . . . . . . . . 131

7.1 .1 Documents . . . . . . . . . . . . . . . . . 131

7.1 .2 IR Systems . . . . . . . . . . . . . . . 132

7.1 .3 Architecture of ANS . . . . . . . . . . . . . 132

7.1.4 The Information Network . . . . . . . . . . . . . . 132

7.1 .5 The Access Problem . . . . . . . . . . . . . . . 133

7.1 .6 Hypermedia in ANS . . . . . . . . . . . . . 133

7.1.7 Browsing the Information Network . . . . . . . . . . . . 133

7.1 .8 Navigation . . . . . . . . . . . . . . . . 133

7.2 The Evaluation . . . . . . . . . . . . . . . . . . . . 134

7.2 .1 IR System Evaluation . . . . . . . . . . . . . . . . 134

7.2 .2 Procedure for ANS Evaluation . . . . . . . . . . . 134

7.2 .3 Performance of ANS . . . . . . . . . . . . . . . . 137

7.3 General Conclusion . . . . . . . . . . . . . . . . . . . . . . 139

7.4 Future Research . . . . . . . . . . . . . . . . . . . . 140

APPENDIX: Questions for the Test Persons $\quad 147$

$\begin{array}{lr}\text { References } & 149\end{array}$

$\begin{array}{ll}\text { Index } & 165\end{array}$

$\begin{array}{ll}\text { Summary } & 169\end{array}$

$\begin{array}{lr}\text { Samenvatting } & 173\end{array}$

$\begin{array}{ll}\text { Curriculum vitae } & 177\end{array}$ 


\section{Preface}

Nowadays most research is performed in teamwork and, hence, also my research has been made possible by many people. In this preface, I would like to acknowledge the various contributions.

Most of all, I am indebted to my supervisors; they initiated me into doing scientific research. I am grateful to Arie Hasman for his inspiring enthusiasm, his ideas, and his patience. Jaap van den Herik taught me the importance of details and to aim at perfection. Jan Dietz' equanimity has been an example for me that prevented me (for a long time) from becoming overburdened.

The research reported in this thesis is part of a larger project, the ARCHIMEDES project. I have pleasant recollections of the cooperation within the project. I thank Jaap Hage, Arno Lodder, Ruud van der Pol, Georges Span, and Bart Verheij for the good teamwork and the many ideas that are incorporated in the ARCHIMEDES Network System (ANS). From the colleagues mentioned I singled out Maarten van der Meulen. I would like to recognize him in particular for his programming efforts, which turned out to be pivotal for ANs. His wife Inge Uljee, although not a member of the project team, helped dealing with some small, yet significant MS Windows programming peculiarities.

Moreover, I thank the many people who gave generously of their time and intellect to the design and the execution of the evaluation experiments. Here I single out Cees van der Vleuten and Hetty Snellen who advised on the experimental design. I thank the following persons for acting as domain experts: G.G.M. Essed, J.W.J.L. Stapert, and G.W. Wesseling (suggesting the search questions and making relevance judgements) and L.L.H. Peeters, F.A.J.M. van den Wildenberg, and G.P.M. ten Velde (making relevance judgements). The test persons were students, who I thank as a team for their contributions to the experiments. Arnold Kester guided me through the pitfalls of statistics; Aimée Herczog's assiduity kept me going when I was about to give up on SPSS.

I express my gratitude to my colleagues at the Department of Medical Informatics for their support. In particular, I mention Patrick Ahles for his help as TEXnician, Hans Blom for his critical questions, Jan Talmon for his ideas, Huibert Tange for his SPSS assistance, and Marieke Vissers for her advice concerning medical 
terminology. Besides, I thank Raphaël Panhuysen for his collaboration concerning the Paleopathology Information Network.

Finally, I thank my family and my friends for their confidence and patience over the years. They now see the result, which I dedicate to the memory of my mother.

\section{Acknowledgements}

The research reported in this thesis was partly financed by the Foundation for Knowledge-Based Systems (SKBS) as part of the B3.A project. SKBS is a foundation with the goal to improve the level of expertise in the Netherlands in the field of knowledge-based systems and to promote the transfer of knowledge in this field between universities and business companies.

The research was performed at the Department of Medical Informatics within an organizational affiliation with the Research Institute for Extramural and Transmural Health Care (Institute ExTra), a participant in The Netherlands School of Primary Care Research (Graduate School CaRe), which was acknowledged by the Royal Dutch Academy of Arts and Sciences (KNAW) in 1995. The research has been carried out also under the auspices of SIKS, the Dutch Graduate School for Information and Knowledge Systems. 


\section{Chapter 1}

\section{Introduction}

Johann Wolfgang von Goethe (1749-1832) was probably the last man who deserves the epithet homo universalis. He was a poet, dramatist, novelist, painter, statesman, and scientist. Scientific disciplines of his interest were anatomy, botany, osteology, chemistry, mineralogy, meteorology, geology, optics, and the history and philosophy of science. He also studied law and music (Brockhaus, 1969; Wells, 1972; Amrine and Zucker, 1987). At the beginning of the nineteenth century it was still possible to make meaningful contributions to multiple scientific disciplines while performing only part-time research. However, in the nineteenth century, the development of science in general led to more publications per year than a person could deal with. As a consequence, scientific disciplines split up into subdisciplines and scientists narrowed their interests so as to be able to keep up to date. Even so, it was not possible to read every publication. In the course of the twentieth century the scientific information stream aggravated. Fortunately, with the advent of computers it became soon possible to search for publications on specific topics, thereby reducing the need for scientists to follow everything that was newly published. The computer programs in question are called information retrieval systems. Salton and McGill (1983, p. xi) define an information retrieval (IR) system as "a system used to store items of information that need to be processed, searched, retrieved, and disseminated to various user populations." Despite of the research carried out in IR, we find ourselves overwhelmed with information, both because of the ever growing amount of digitally available information and the imperfection of the existing IR systems.

This thesis is about IR systems. Chapter 1 provides an overview of the topic to be investigated. Sections 1.1 and 1.2 discuss why it is important to find information and why it is so difficult. Then Section 1.3 describes two ways in which one can search for information: querying and browsing. They are an important part of the ARCHIMEDES project, which underlies the research presented. This project is outlined in Section 1.4. The problem statement and hypotheses are presented in Section 1.5 and finally Section 1.6 gives an overview of the following chapters. 


\subsection{Finding Information}

To establish the importance of finding information and the role of IR systems, we focus on scientific information. The people who deal with scientific information are the producers of the information (1.1.1), the users (1.1.2), and the students (1.1.3). Below we discuss these groups according to the distinction given.

\subsubsection{Producers of Information}

Scientists produce scientific information: so as to do this, they must know how their current research is related to research world wide. Since any scientific publication contains references to related work, it is important for a scientist to find and access all publications concerning the field of his ${ }^{1}$ research. This is the most obvious application of IR. Oddy et al. (1992) describe several generic research scripts and claim that IR systems should support the user in following these research scripts. We mention the steps in the scripts that require IR actions: making sure the research has not been carried out before, exploring the field, compiling a literature review, consulting the literature to solve a deadlock in the research, writing up the research results in a paper, and finding out when and how to publish the paper.

\subsubsection{Users of Information}

As an example of the application of scientific information we look at medicine. Physicians use medical scientific information. They usually find their information through Medline, the largest biomedical literature database in the world. We discuss three studies that establish the importance of IR for physicians.

In clinical practice it often happens that physicians face unanswered clinical questions. Chambliss and Conley (1996) collected such questions over a period of six months and searched in Medline and in textbooks to answer them. Almost one question per day per physician was collected. More than a third of the answers had a salient influence on the physician's practice. Nevertheless, the physicians stated that reading entire articles was too time consuming; they preferred direct answers to their questions.

Lindberg et al. (1993b) interviewed workers at a hospital who issued a total of 1158 searches. The authors conclude that the results of the searches had a positive influence on the choice of diagnostic tests, the diagnosis, the development and implementation of a treatment plan, the physician-patient relationship, the modification of a patient's health behaviour, and the deliberations with third-party payers (e.g., insurance companies). In eight cases, lives of patients were saved.

Klein et al. (1994) compared hospital costs of clinical cases where literature searches were performed during hospitalization with clinical cases where no searches

\footnotetext{
${ }^{1}$ For the sake of readability we will use from now on only one gender: we have chosen the male gender of pronouns in all cases where the person referred to could evenly well be female.
} 
were made. It is concluded that use of the medical literature results in reduced costs and a shorter length of stay. The explanation is that via the medical literature new diagnostic tests and therapies become available, as well as information about when not to use particular tests or therapies.

\subsubsection{Students}

Students learn from the scientific information. The educational system at Maastricht University, called problem-based learning, stimulates students to make their own selection of literature. Instead of passively following lectures, students have to formulate questions and find the answers in the literature. Some literature is made directly available, other literature can be found using several IR systems (e.g., the university library's IR system and Medline). Finding information is valuable also beyond problem-based learning. For instance, third-year medical students at the University of Michigan have to write a thesis using primary literature. They receive training in searching Medline. Schwartz and Schwartz (1995) report on a survey among these students concerning the use of Medline. The authors conclude that almost all students made use of Medline and that the majority found it helpful in preparing their theses.

Here we would like to establish that many students (and seasoned researchers too) disregard the difference between data and information. Information is usually considered as interpreted data. When information is placed in a semantic context (i.e., it has a meaning assigned) it can be considered as knowledge (Van den Herik, 1997). In this thesis, we will not make a distinction between data, information, and knowledge. For our purpose it suffices to find information; whether it has a meaning and whether it is applied, is not our concern.

\subsection{Finding the Right Information}

Finding information is not a problem. The problem is to find the right information amidst the huge amounts of available information. A perfect IR system would retrieve precisely the relevant documents; no more, no fewer. Several studies have shown that the effectiveness of existing IR systems is rather inadequate. The most-acknowledged study is by Blair and Maron (1985), even though its publication is more than a decade ago. Its surprisingly disappointing results and the fact that it was the first evaluation of a large commercial information system have made this study renown. A commercially available IR system with almost 40,000 documents was used. The test persons thought they had found approximately three-quarters of all relevant documents. In reality, they had found no more than one fifth. ${ }^{2}$ Shaw Jr. et al.

\footnotetext{
${ }^{2}$ The system used, called STAIRS, was a full-text IR system. This means that the system contains all the words of the documents, as opposed to only a selection. Blair and Maron (1985) claim that the conclusions of the study apply to full-text IR systems in general. Full-text IR is
} 
(1997) surveyed over 100 IR papers published in the period 1985-1994. The authors conclude that retrieval effectiveness was generally mediocre. Rarely more than half of the relevant documents were retrieved and rarely more than half of the retrieved documents were relevant.

Why is IR so difficult? In the following, we discuss six kinds of difficulties that may arise while searching for information. These difficulties concern the information need (Subsection 1.2.1), the communication between the IR system and its users (Subsection 1.2.2), the communication between humans (Subsection 1.2.3), knowledge (Subsection 1.2.4), indexing (Subsection 1.2.5), and reliability (Subsection 1.2.6).

\subsubsection{The Information Need}

The information need is the mental image that a user has of the information he wants to retrieve. If the user only needs a fact as answer (e.g., how much bile does the average human liver produce per day?) the information need is clearly determined. Often, a problem-oriented information need is not clear. An example is: "I need information on the production and storage of bile." The boundaries of this information need are not clear and may vary with time (Frants et al., 1996). If the user does not know the precise boundaries of his information need, how can the IR system determine exactly which documents fulfil the information need? The severity of the problem depends considerably on the number of documents that the IR system contains. A vague information need (or a vaguely expressed information need) does not necessarily yield an overwhelming number of retrieved documents if the IR system contains only a small number of documents. Conversely, for an IR system with many documents a vague information need probably results in more documents than manageable for the user. An analogous situation occurs if the IR system covers the topic of the query only partly (or not at all).

\subsubsection{Communication between the IR System and its Users}

Because of their different natures there is a communication gap between the IR system and its users. On the one hand, there are IR systems that process human language. However, the possibilities in the domain of natural-language understanding are still limited. On the other hand, the user can communicate with the IR system in a formal language. An utterance in such a language is called a query. It consists of one or more key terms, possibly accompanied by logic connectives. After supplying a query, the IR system retrieves the documents that satisfy the query. After inspecting the documents, the user may decide to revise the query. A series of queries issued to satisfy a particular information need is called a search. It is often troublesome for humans to adhere to a formal language. Reisner (1988) remarks that

further discussed in Section 2.3.7. Blair (1996) discusses the impact of the 1985 article. 
formal languages for database systems are "complex and involve cognitive activities (learning, understanding, remembering) rather than only physical and perceptual ones."

The poor communication between the IR system and its users hampers effective retrieval: if the user cannot make clear his information need to the IR system, it cannot find the proper documents.

\subsubsection{Communication between Humans}

Humans use IR systems to retrieve documents that were composed by humans. It may very well be that the human who searches documents uses other key terms ${ }^{3}$ to designate particular concepts than the human who composes documents. Even worse, these humans may have different perceptions of a particular concept. In both cases documents that are irrelevant may be retrieved and documents that are relevant may not be retrieved. Hence we can speak of an implicit communication problem between humans.

\subsubsection{Knowledge}

Parsaye et al. (1989, Chapter 6) distinguish five kinds of knowledge that are important for IR. We discuss each of them and state why there is a problem.

Procedural knowledge for using the IR system: This kind of knowledge concerns the commands that the user can give and the procedures he can follow (e.g., combining two key terms with the AND operator results in the intersection of the documents that would be found with the individual key terms). While working with a system, the user builds a mental model thereof. The more complex a system is, the more misconceptions the mental model may contain and the less effective the user can work with the system. Having reviewed several experiments, Carroll and Olson (1988) conclude that even experienced users do not necessarily have a complete and correct mental model of the system they work with.

Domain knowledge: When a query has to be revised because the results are unsatisfactory, knowledge of the domain of the documents becomes important. For instance, a query that resulted in too many retrieved documents has to be narrowed. This can be done by substituting a general key term by a more specific one. To choose the new key term requires knowledge of what concepts exist in the domain, how they are related to each other, and by what key terms they are designated. Either the user must have this knowledge,

\footnotetext{
${ }^{3}$ Throughout this thesis, we use 'key term' instead of the more commonly used 'key word', because it indicates better that it may consist of multiple words. For instance, Graves' Disease therapy can be considered as one key term.
} 
which may not be the case for a domain novice, or the computer must have it, which makes the IR system domain dependent. Furthermore, representation of domain knowledge is not a trivial task.

Is it really important to use domain knowledge? Bodner and Song (1996) state that the success of query-oriented IR depends on the size of the query. Short queries do not provide sufficient information to the IR system to distinguish relevant documents from irrelevant ones. However, users often enter short queries (in a study by Rose and Stevens (1997) $53 \%$ of the queries consisted of one key term). This is caused by the users' lack of knowledge of the domain discussed in the documents. A secondary cause is that users do not want to formulate long queries. Hence, Bodner and Song conclude that a system is needed that supports the user with additional domain knowledge. This conclusion is supported by Howard (1992): he found that users with much domain knowledge can deal better with complex information needs than users with little domain knowledge (the users were equally experienced in searching). However, there is no difference between the groups for information needs that are complex.

Strategic knowledge of search request formulation: For the formulation of a query, strategic knowledge of query formulation is needed. This concerns the impact of the commands that constitute the query (e.g., combining key terms with the AND operator yields less documents than the separate key terms). It should not be confused with the knowledge of the possible commands and what they do (that is procedural knowledge, mentioned above). Novice users have limited strategic knowledge of search request formulation; the knowledge is often incomplete and contains misconceptions. For experienced users this knowledge is well-developed.

Knowledge of the used construction and indexing policies: Indexing is the process of assigning key terms to documents so as to describe which documents contain which concepts. To deploy an optimal search strategy the user needs to know and understand how documents are indexed. For instance, are all words of the documents indexed or only the title words, how often are updates made, what spelling conventions are followed? Only advanced users can be expected to have this knowledge.

High-level search knowledge: This kind of knowledge is concerned with strategy, that is, a predefined plan describing how to develop one query out of another based on the query's results. High-level search knowledge differs from strategic knowledge of query formulation in that the latter pertains to the formulation of a single query. An example of a strategy is pearl growing: once a relevant document is retrieved, its associated key terms or authors' names are exploited to find other documents. The resulting relevant documents can be 
used recursively for pearl growing. Novice users have little high-level search knowledge and most IR systems do not have it at all.

\subsubsection{Indexing}

The process of assigning key terms to documents is called indexing. The communication difficulties mentioned in Sections 1.2.2 and 1.2.3 also apply to indexing - be it human-human communication if the indexing is performed by humans, or humancomputer communication if the indexing is performed automatically. Additionally, a human indexer may not be consistent (using different key terms for similar documents), may not be complete (erroneously omitting a key term from a document), and may not be correct (assigning a wrong key term to a document). Marson and Chadwick (1996) studied how easy documents about a particular subject (randomized controlled trials in epilepsy) could be found in Medline. They conclude that several documents were not found because the indexers had not assigned the proper key terms. Another reason was that the titles and abstracts did not contain adequate descriptions of the document contents.

\subsubsection{Reliability}

Most likely, the user requires that the retrieved information is reliable. Reliability is a problem where the information source is a public one, such as the Internet. On the Internet, anyone can make arbitrary statements. There is no regulating authority who verifies the information. Thus, Internet users cannot be sure that the facts in a particular document are true and hence that the document is reliable.

\subsection{Querying versus Browsing}

In the previous section we mentioned the subject of information need. The behaviour of a user of an IR system depends on various factors, such as experience with IR systems, domain knowledge, and time constraints. A significant factor is the articulateness of the information need. The behaviour of a user with a clear and well-defined information need will differ from the behaviour of a user with a vague information need. The concept of querying is generally associated with the former information need, the concept of browsing with the latter one. When querying, the user describes the information he is looking for. When browsing, the user navigates through the information until he recognizes the information he is looking for. We concentrate on browsing. In this section we mention three classifications of browsing behaviour. Moreover we show that browsing is not exclusively suitable for vague information needs. 


\subsubsection{Cove and Walsh' Browsing Types}

Cove and Walsh (1988) distinguish three browsing types:

Search browsing: The user browses with a specific goal in mind.

General-purpose browsing: The user browses information sources regularly because they probably contain relevant information.

Serendipity browsing: The user browses purely at random.

\subsubsection{Chang and Rice's Browsing Dimensions}

Browsing is especially associated with hypertext systems. ${ }^{4}$ Chang and Rice (1993) give a systematic overview of browsing in various disciplines. For instance, television channel 'zapping' and 'wayfinding' (moving through a space to reach a destination) can be considered as browsing. Moreover, properties of browsing in hypertext apply also to browsing in other disciplines. Chang and Rice argue that there is a whole spectrum of browsing and not a few discrete browsing types. The browsing types of Cove and Walsh (1988) can be considered as a subset of the browsing types of Carmel et al. (1992). Six dimensions are identified along which browsing types can be classified. The dimensions all contain a continuous spectrum:

Purpose: Is there a reason to browse? This dimension ranges from intrinsic (e.g., recreation) to extrinsic (e.g., informational).

Goal: Is there a goal to be fulfilled? This dimension ranges from non-goal-directed to goal-directed.

Content knowledge: Is the browsing destination known? This dimension ranges from non-content-specific (e.g., a physical item, where the person browsing is not interested in the content, but the item itself) to content-specific (e.g., information).

Structure knowledge: Is the route to the desired items known? This dimension ranges from non-path-specific (e.g., a physical path) to path-specific (e.g., meta-information).

Location knowledge: Is the browsing confined to a specific location? This dimension ranges from non-location-specific to location-specific (e.g., a position on a bookshelf).

Resource focus: Is the browsing focussed at the content of the destination or at the path to the destination? This dimension ranges from content (e.g., a text in a book) to path (e.g., the organization of chapters in a book).

\footnotetext{
${ }^{4}$ Carmel et al. (1992) provide an adequate overview of hypertext systems.
} 
Search browsing of Cove and Walsh (1988) can be expressed with goal-directed as goal dimension and any value for the other dimensions. Cove and Walsh' generalpurpose browsing and serendipity browsing both correspond with non-goal-directed as goal dimension and any value for the other dimensions.

\subsubsection{Waterworth and Chignell's Information Exploration Classification}

Instead of merely classifying browsing types or identifying browsing dimensions, Waterworth and Chignell (1991) propose a unified classification for information exploration, which encompasses both browsing and querying. Unfortunately, they use the term 'navigating' where we use 'browsing' and use the term 'browsing' for 'searching with an unclear information need'. Henceforth, we enclose 'browsing' in single quotes where it is used in the latter meaning. Moreover, they define 'querying' as 'searching with a clear information need'; in the following, single quotes around 'querying' signify that meaning. Waterworth and Chignell define three dimensions for information exploration:

Structural responsibility: This is the dimension of who is concerned with the structure of the information (i.e., the organization of the information and the way information items are linked to each other). When 'browsing', the system is aware of the structure and the user controls the search process. When 'querying', the user must be aware of the structure and the system controls the search process.

Target orientation: This dimension concerns the cognitive state of the user: when 'querying', the user has a clear information need. When 'browsing', the user does not have a clear information need.

Interaction method: This dimension differentiates between descriptive interfaces, where the user describes the desired information (a query), and referential forms of action, where the system offers alternatives and the user selects what is desired.

From the first two dimensions four types of IR can be identified:

Navigational 'browsing': The user moves through the information without a clear information need.

Navigational 'querying': The user has a clear information need, but still navigates through the information.

Mediated 'browsing': The user finds information by querying the system, browsing through the results, and reformulating the query after reading the results. 
Mediated 'querying': The user finds information by querying the system; the user reformulates the query on basis of the number of retrieved documents (which he classifies as, for instance, too many, too few, or manageable).

Navigational 'querying' is equivalent with search browsing of Cove and Walsh (1988), and Chang and Rice's (1993) extrinsically motivated, goal-directed browsing. Navigational 'browsing' corresponds with Cove and Walsh' general-purpose browsing and serendipity browsing, and Chang and Rice's non-goal-directed browsing.

\subsubsection{Conclusions on Querying versus Browsing}

Subsections 1.3.1 through 1.3.3 mentioned three partly overlapping classifications of browsing behaviour. We conclude that browsing is used with various information needs, ranging from vague or empty to clear needs. Querying and browsing complement each other, but browsing can be sufficient for various information needs. Searching with a vague information need corresponds with Cove and Walsh' (1988) general-purpose browsing and serendipity browsing, Chang and Rice's (1993) intrinsically or extrinsically motivated non-goal-directed browsing, and Waterworth and Chignell's (1991) navigational 'browsing'. Searching with a clear information need corresponds with Cove and Walsh' search browsing, Chang and Rice's extrinsically motivated, goal-directed browsing, and Waterworth and Chignell's navigational 'querying'.

The disadvantage of searching by browsing is that it may be laborious. Its advantage lies in the division of roles between user and computer: the initiative is by the human being, the computer does the hard work. Human beings are better in recognizing relevant information than in describing it. According to Shneiderman (1992, Chapter 2) human beings are good at adapting to a situation, making subjective evaluations and changing an approach if the original fails. Computers are good at storing and recalling quantities of coded information.

Apart from query-oriented search and browsing-oriented search Hawkins (1996) also distinguishes grazing. In this kind of search, the user issues a search request once, and then relevant information is delivered on a regular schedule. Grazing is important for commercial applications. It is not further discussed in this thesis.

\subsection{The Archimedes Project}

The ARCHIMEDES project was carried out from 1992 to 1996 at the departments of computer science, medical informatics, metajuridica and management sciences of Maastricht University. It was part of the SKBS B3 project (1991). The goal of the ARCHIMEDES project was to provide users with a uniform search and access mechanism to multimedia information. The previous sections of this chapter discussed the issue of searching for information. A related issue is the access to information. It 
is defined as retrieving (i.e., locating and transporting) the information found and subsequently transforming the form in which the information is stored into a form perceptible to the human senses. Different types of information require different transformation methods. Therefore, access is an important issue where multimedia information is involved. Unfortunately, in IR, 'to retrieve' is often used with the meaning 'to find'. Throughout this thesis both meanings are used; from the context it should be clear which meaning is intended.

The approach chosen to improve search and access is to combine four kinds of meta-information in one structure, called the Information Network. The metainformation is information about (1) the domain discussed in the documents, (2) the contents of the documents, (3) the relations between the documents, and (4) the locations of the documents. The Information Network can be considered as an extension of a semantic network (see Maida and Shapiro (1982) for an account on semantic networks).

The user can search the Information Network using a query tool or a browser tool. The advantage of the browser tool over traditional browsers is that browsing is brought to a higher level: the user does not browse information but browses metainformation. The browser tool is especially suited for a graphical user interface. Shneiderman (1992, Chapter 11) mentions several advantages of such an interface. Firstly, it may be more familiar for the user because it is closer to the real world. Secondly, there are intuitive ways of displaying relations and stressing important parts. Thirdly, with a pointing device and rapid feedback from the computer the user can work efficiently.

The ARCHIMEDES approach addresses five of the problems identified in Section 1.2: (1) the problem of human-computer communication (by performing browsing-oriented search instead of query-oriented search), (2) the problem of human-human communication (by using a concept-oriented system instead of a string-oriented one), (3) the problem of missing or faulty procedural knowledge (by using an intuitive user interface), (4) the problem of missing or faulty strategic knowledge of search request formulation (by using an intuitive user interface), and (5) the problem of missing or faulty domain knowledge (by giving the domain representation a central position in the system).

\subsection{Problem Statement and Hypotheses}

The ARCHIMEDES project addresses search and access with the Information Network, which can be queried and browsed. In this thesis we focus on search by browsing the Information Network. Section 1.3 showed that browsing may be sufficient for various information needs. The following two hypotheses are formulated:

1. The meta-information to be browsed can be represented in an Information Network: a structure that contains references to information, combined with a domain representation. 
2. Searching by browsing the Information Network has a higher performance than using existing search programs.

Our problem statement reads as follows:

Is it possible to provide facilities for searching various information items by allowing the user to browse in a graphical way through metainformation, making it an improvement over existing methods?

With 'various information items' we mean text documents of all kinds: text fragments, journal articles, abstracts, books, hypertext, and so on. Multimedia information is also allowed, but is treated like textual information in the sense that each multimedia document is supposed to have key terms and an identifier. The problem of automatically indexing is not addressed.

Our goal was to realise an architecture that allows high-performance search to information by graphically browsing meta-information. We identified two subproblems.

Firstly, how can a graphical meta-information browser be realised? To solve this subproblem we had to answer the following research questions.

- What is, in the context of the ARCHIMEDES project, a document? How and when are documents distinguished from one another? What types of documents exist?

- What are characteristics of existing IR systems? Which of the characteristics are of interest for the ARCHIMEDES system?

- What should the architecture of the ARCHIMEDES system be?

- How should the Information Network be defined? How should it be specified?

- How can uniform access to the retrieved information be realised?

- How do the concepts of hypertext and hypermedia fit into ARCHIMEDES?

- How can the Information Network be visualized and browsed?

- How can disorientation of the user while browsing through the Information Network be prevented?

Secondly, is the performance of the ARCHIMEDES system better than the performance of existing search programs? To solve this subproblem we had to answer the following research questions:

- How does one evaluate an IR system?

- How to evaluate the ARCHIMEDES system? 
- How does the ARCHIMEDES system perform compared to other systems?

In the evaluation, a distinction has to be made between users with little domain knowledge and users with much domain knowledge, because they may react differently on the domain knowledge in the Information Network.

\subsection{Thesis Outline}

The outline of this thesis is as follows. Chapters 2 through 4 address the first series of research questions. In Chapter 2 an overview of the IR domain is given. Several characteristics of IR systems are discerned, and for the ARCHIMEDES system a selection of required characteristics is made. Chapter 3 starts describing the various kinds of information, and the notion of a document. Subsequently, the architecture of the ARCHIMEDES system is discussed. The query tool introduced in that chapter is further developed in a related research project (Van der Pol, 1996). Because the emphasis in our research shifted to the browsing part, the query tool is not further discussed. The ARCHIMEDES system actually implemented is described in Chapter 4. It discusses the visualization algorithm in detail and presents several implemented Information Networks.

Chapters 5 and 6 address the second series of research questions: the evaluation of the ARCHIMEDES system. To identify difficulties that may arise during the evaluation Chapter 5 reviews the literature on this subject. The remainder of the chapter describes the protocol of the evaluation. We decided to do a laboratory experiment with a medium-sized document collection, where the ARCHIMEDES system is compared with a commercially-available IR system. The results are presented in Chapter 6. Finally, Chapter 7 draws conclusions on the hypotheses, the problem statement, and the research questions formulated in Chapter 1 . The results are discussed, and directions for future research are given. 



\section{Chapter 2}

\section{Information Retrieval: An Overview of System Characteristics}

This chapter is a slightly adapted version of: Wiesman F., Hasman A., and Van den Herik H.J. (1997). Information retrieval: an overview of system characteristics. International Journal of Medical Informatics, Vol. 47, No. 1-2, pp. 5-26.

This chapter provides an overview of the characteristics distinguishing among IR systems. The overview deals with 23 IR systems described in sufficient detail for an adequate comparison. We do not aim at making quantitative statements on the IR systems' characteristics but at listing characteristics for comparing all possible IR systems. The choice of the 23 systems does not reflect the complete IR market. Some emphasis has been put on systems that apply artificial-intelligence techniques. Nevertheless, this chapter provides a clear picture of the current techniques and developments in IR.

The first section briefly introduces the terminology used in IR. Section 2.2 focusses on one characteristic: the IR model. It describes the four most prominent IR models. Twelve additional characteristics of IR systems are discussed in Section 2.3. Section 2.4 presents an important area for the application of IR: the World Wide Web. Finally, Section 2.5 provides conclusions on IR systems in general and the implications for the ARCHIMEDES IR system.

\section{$2.1 \quad$ Background}

The function of an IR system is to lead its users to those documents that will best enable them to satisfy their information need (Robertson, 1981). The documents 
can be of various kinds: text, sound, images, video, and so on. 'Real-world documents' can be stored in a computer at different levels of detail: as reference only (for instance, for this chapter: published - in a slightly different form - in International Journal of Medical Informatics, 1997, Vol. 47, No. 1-2, pp. 5-26), as reference together with an abstract, or with the entire contents. All IR systems have a way of describing the document contents: the document representation. This may be a list of key terms, a conceptual graph, a frame, or any other representational form.

The user communicates his information need to the IR system by formulating a query. Queries usually exist of a list of key terms, which may be combined by connectives, such as AND and oR. Other possibilities are queries formulated in a natural language or in graphically-oriented query languages. These types of queries are discussed in Subsections 2.3.4 and 2.3.5. Once a query is formulated, the IR system determines which document representations meet the query (the matching procedure), and reports this to the user. The user may decide to reformulate the query if the results are not satisfactory.

The following two quantities together form a measure for the effectiveness of an IR system (Kent et al., 1955):

Recall: The part of the relevant documents that is actually retrieved

Precision: The part of the retrieved documents that is actually relevant

It is amenable to achieve a high recall at the cost of precision. Likewise, a high precision can be attained at the cost of recall. The problem is to balance recall and precision. Buckland and Gey (1994) analyse this problem theoretically. Section 5.1 reports on the literature on IR system effectiveness.

The generation of document representations is called indexing. The quality of the indexing process is defined as the degree to which the representation reflects the content of the document. A high quality is vital for a proper operation of an IR system. It depends on the document representation form whether the indexing can be performed automatically. Simply assigning key terms can be done automatically with the use of statistical techniques. Computers do so with satisfactory results (Harman, 1994, 1995). For each word occurring in the document the frequency is determined. Common words (called stopwords), such as the, a, in, of, from, are eliminated. On the basis of its frequency each word is assigned a weight. For the weighting process different formulas are in use.

More complex representations that truly reflect the content of a document are still to be constructed manually. These encompass advanced formalisms such as frames and first-order logic, in which documents are not represented at a global level but at paragraph or sentence level. The advantage is the greater expressive power of the query language. Additionally, by representing facts from documents a query system can answer questions about the contents of documents. This is called a question-answering system. The manual construction of document representations required by an advanced representation formalism imposes restrictions on the size of 
the system. An advanced system that uses complex representations is TOPIC (Hahn and Reimer, 1988).

Multimedia IR systems are still in their infancy. Non-textual media IR faces the problem of making their semantics intelligible to the computer; this requires image and speech recognition techniques. Most current systems do not automatically extract content representations, but merely features (e.g., the colours used in an image). Gupta and Jain (1997) provide an introduction to the subject. Aigrain and Longueville (1991) and Shneier and Abdel-Mottaleb (1996) report on IR with images and Smoliar and Zhang (1994) conducted experiments on IR with video. For this chapter, we assume that information consists of documents containing text.

\subsection{The Information Retrieval Model Characteristic}

When looking at an IR system the question arises: what IR model has been used? An IR model specifies a document representation, a query representation, and a matching procedure. The majority of IR systems is based on the Boolean model. The vector model is the most frequently used in experimental environments (Shaw Jr. et al., 1997). Both will be discussed here. Other models are: the probabilistic model, the connectionistic model, the cluster model, the rule-based model, the fuzzy-logic or fuzzy-set model, and the semantic model. We elaborate on the Boolean model and vector model since they have an extensive record.

The examples in this chapter will be based on the following case: Annette has only little knowledge of IR and is looking for information on gall bladder surgery. She is especially interested in scientific publications on complications that may occur as a result of one particular operation: choledochojejunostomy. In this operation a direct connection (stoma) is made between the common bile duct (ductus choledochus) and the small gut (jejunum).

\subsubsection{The Boolean Model}

The most popular model for building IR systems - and especially commercial ones - is the Boolean model. In this model a document is represented by a set of key terms: chosen from a fixed set of key terms, or, possibly automatically, from the documents themselves. Queries and documents can be matched by checking for each document whether the associated key terms satisfy the query. A query consists of a list of key terms connected by logic connectives, such as AND, OR, and NOT.

For our sample user the query may read:

(ductus choledochus OR common bile duct) AND surgery AND complication

This query can be formalized as A AND B AND C. Compared to the query A AND $B$ it may be expected that the former query selects fewer documents. This touches upon a well-known problem of the Boolean model: the more key terms are involved 
with AND operators, the fewer documents are retrieved. The decrease of documents retrieved can be very drastic. The cause is that the AND operation imposes severe restrictions on the presence of the key terms. Vice versa, leaving out one or two key terms may result in a (too) large set of retrieved documents. An analogous but converse reasoning holds for the oR operator: the more key terms are added, the larger the set of retrieved documents.

Next to this problem the Boolean model has three drawbacks (Crouch et al., 1989; Salton, 1991):

- A good query is difficult to formulate, especially for IR novices, since the impact of complex combinations of the operators is hard to grasp.

- The relative importance of the key terms for a query cannot be specified.

- Arranging the retrieved documents in order of relevance is difficult. This is further discussed in Subsection 2.3.12.

Of course, the Boolean model also has advantages:

- The Boolean model can be easily implemented and has low computational cost (Salton and McGill, 1983).

- Its query language is more expressive than that of other models (Croft and Thompson, 1987).

- The model is fit for users who know exactly what they are looking for (Turtle, 1993).

The following seven systems use the Boolean model: WinSpirs ${ }^{1}$, Consens and Medezon (1989), Smith et al. (1989), Gauch and Smith (1991), Humphrey (1992), Yoon et al. (1992), and Charoenkitkarn et al. (1995).

\subsubsection{The Vector Model}

In a vector-model IR system containing $n$ key terms, an $n$-dimensional space is defined such that each axis is associated with a different key term. ${ }^{2}$ Every key term has a weight. Documents and queries are represented by vectors in that space. A document vector is an $n$-tuple in which every coordinate is represented by the weight of the corresponding key term. The higher the weight, the more important the key term is

\footnotetext{
${ }^{1}$ WinSpirs is a commercially available in system from Silverplatter. No literature reference is available, so we refer to Silverplatter's home page: http://www.silverplatter.com/

${ }^{2}$ Instead of key terms, Cavnar (1995) associates with each axis a four-letter string. The document is considered as a sequence of four-letter strings (e.g., retieval is stored as reti, etie, tiev, ieva, eval). Thus, only parts of words are indexed. The cost of redundancy is made up because firstly the system is robust against spelling mistakes in the query (as in the example) and secondly, the system is language independent because there is no need for a stopword list.
} 


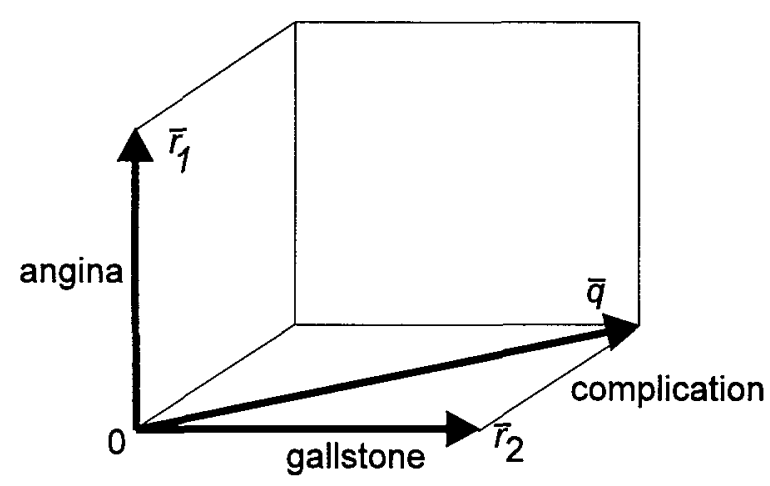

Figure 2.1: Vector space with three key terms.

to the document associated with the vector. A query vector is defined analogously, only the weights express the importance of the key terms for the user. Usually a 1 is used for desired key terms and a 0 for undesired key terms.

The weights are assigned on the basis of word frequency. There are many ways of doing this (see e.g., Salton and Buckley, 1988); we present a well-known example (cf. Salton, 1991). The weight of a key term $w$ in document $i$ is defined as:

$$
\operatorname{weight}(w, i)=\frac{f_{w i} \log \left(N / n_{w}\right)}{\sqrt{\sum_{k=1}^{W_{i}}\left(f_{k i}\right)^{2}\left(\log \left(N / n_{k}\right)\right)^{2}}}
$$

where $f_{w i}$ is the frequency that key term $w$ occurs in document $i, N$ the number of documents, $n_{w}$ the number of documents in which key term $w$ occurs, and $W_{i}$ the total number of key terms in document $i$.

The weight is proportional to the number of times the key term occurs in the document. Key terms that also occur in other documents are relatively unimportant, hence the weight is adjusted by a factor $\log \left(N / n_{w}\right)$. The length of a vector is thus dependent on the occurrence and frequency of the key terms involved. To avoid the influence of their length the vectors are normalized (in the denominator).

There are several ways of calculating the similarity between a document vector and a query vector. One of them is the normalized dot product of the two vectors. As a case in point we take a universe of three key terms (see Figure 2.1):

$$
\text { <gallstone, angina, complication> }
$$

We assume that only two documents are present. Document 1 is on complications with angina and document 2 on complications with gallstones. Since the term complication occurs in both (all) documents the weight of complication is 0 in both documents. This implies, after normalization, the following document representations 
for document 1 and document 2 , respectively:

$$
\bar{r}_{1}=\left(\begin{array}{l}
0 \\
1 \\
0
\end{array}\right) \quad \bar{r}_{2}=\left(\begin{array}{l}
1 \\
0 \\
0
\end{array}\right)
$$

We now want to retrieve documents with the key terms gallstone and complication by the query vector

$$
\bar{q}=\left(\begin{array}{l}
1 \\
0 \\
1
\end{array}\right)
$$

For a query vector $\bar{q}$ and a document vector $\bar{r}$ in a system with $n$ key terms the similarity is:

$$
\operatorname{similarity}(\bar{q}, \bar{r})=\frac{\sum_{i=1}^{n} q_{i} r_{i}}{\sqrt{\sum_{i=1}^{n}\left(q_{i}\right)^{2} \sum_{i=1}^{n}\left(r_{i}\right)^{2}}}
$$

This formula applied on the example results in:

$$
\begin{aligned}
& \operatorname{similarity}\left(\bar{q}, \bar{r}_{1}\right)=0 \\
& \operatorname{similarity}\left(\bar{q}, \bar{r}_{2}\right)=0.7
\end{aligned}
$$

Document 2 scores better than document 1 and therefore matches better with the query than document 1 , hence document 2 is retrieved.

In practice, a universe of $n$ key terms does not mean that each document is described by $n$ weights; the vast majority of the weights will be zero because most documents contain a limited number of key terms. Therefore, only combinations of key terms with weights are stored for weights that are unequal to zero. Thus, the vector model does not require much more memory than the Boolean model, and the response time is also reasonable.

Advantages of the vector model are:

- It is possible to assign weights to key terms in a query (simply by replacing the ones and zeros in the query vector).

- The similarity measure can be used to present the results in order of relevance.

- Many researchers assume that the retrieval results obtained with the vector model are better than those obtained with the Boolean model. This is supported by various experiments (Turtle, 1994; Savoy, 1994). However, in the study of Lu et al. (1996) no differences in effectiveness were found between Boolean and vector systems. An explanation may be that in this study a very large document collection was used.

The vector model does not have the disadvantages of the Boolean model, but it has others (Crouch et al., 1989): 
- Key terms are supposed to be independent.

- In a query no logic relations (such as AND, OR, and NOT) between key terms can be used.

Shaw Jr. et al. (1997) surveyed over 100 IR papers published in 1985-1994, and conclude that from all models the vector model is by far the most frequently used. Six of the systems studied use the vector model: Gordon (1988), Motro (1988), Can (1993), St. Pierre et al. (1993), Bodner and Song (1996), and Rose and Stevens (1997).

\subsubsection{The Probabilistic Model}

The basis for the probabilistic model is the probability ranking principle (Robertson, 1977):

The best possible retrieval results are achieved when documents are shown in the order of their probable relevance to the query.

The task of the IR system is to compute the relevance of a document to a query. This section follows the account given by Salton and McGill (1983, Chapter 3). A query consists of a set of key terms chosen from a fixed universe of key terms. A document contains a set of terms from the same universe:

$$
\text { Doc } \left.=<\text { term }_{1}, \ldots, \text { term }_{t}\right\rangle
$$

A document is retrieved if the following condition is satisfied:

$$
p(\operatorname{Rel} \mid D o c) \geq p(\text { Notrel } \mid D o c)
$$

where Rel means the document is relevant and Notrel that the document is not relevant to the query. With Bayes' rule (Bayes, 1763) this can be rewritten as:

$$
\frac{p(\text { Doc } \mid \text { Rel }) p(\text { Rel })}{p(\text { Doc } \mid \text { Notrel }) p(\text { Notrel })} \geq 1
$$

The IR system orders the retrieved documents using the left-hand side of the inequality. A problem is how to determine the necessary probabilities. One can do this by using document samples of which the relevance is known. If queries consist of only one key term or key terms are assumed independent, the relevance of each query term that matches a document term can be expressed as the following odds-ratio:

$$
w=\frac{r /(R-r)}{(n-r) /(N-n-(R-r))}
$$

where $N$ is the number of documents in the collection, $n$ is the number of documents that contains the term, $R$ is the number of relevant documents, and $r$ is the number of 
relevant documents that contain the term. Given a query $\left\langle w_{1}, w_{2}, \ldots, w_{t}\right\rangle$ with $w_{i}$ is the weight as determined in the Equation 2.3, and a document $\left\langle x_{1}, x_{2}, \ldots, x_{t}\right\rangle$ with $x_{i}=1$ if the key term is present in the document and $x_{i}=0$ if it is not, we can define the similarity function:

$$
\operatorname{similarity}(q u e r y, \text { document })=\sum_{k=1}^{t} x_{k} w_{k}
$$

Kwok (1990) and Robertson et al. (1996), authors of the two systems in this survey that use the probabilistic model, modified Equation 2.3 to include the frequency of the key term in the document. Kwok also included the frequency of the key term in the entire document set.

According to Savoy (1994) the effectiveness of the probabilistic model is clearly better than of the Boolean model, and slightly worse than of the vector model. The probabilistic model has the following disadvantages:

- Key terms are supposed to be independent of each other (as in the vector model).

- There is no method for estimating term relevance at the beginning, when no relevant documents are known.

A special form of the probabilistic model is the Bayesian network. Such a network defines in terms of probabilities how the relevance of a particular key term changes as soon as the relevance of a related key term changes. Because of its suitability for integration with hypertext systems this model is becoming increasingly popular. However, computational complexity is high, which suggests that the model is not fit for larger networks. Savoy and Desbois (1991), Callan et al. (1992), and Turtle (1993) use this model.

\subsubsection{The Connectionistic Model}

Neural networks create a form of connectionism which is also applicable in IR. A neural network consists of a number of simple processing units called neurons. They communicate with each other by sending signals over a large number of weighted network connections. Each neuron does nothing else than receiving signals and computing an output signal that is propagated to other neurons. Normally the neurons are divided into several layers. The input layer is used to offer a problem to the network by activating the appropriate nodes. The resulting activation of the output layer nodes determines the solution. Between these layers there may be other layers, the hidden layers. A network can be trained by adjusting its weights, for instance, as a reaction to the difference between the desired output and the actual one. 


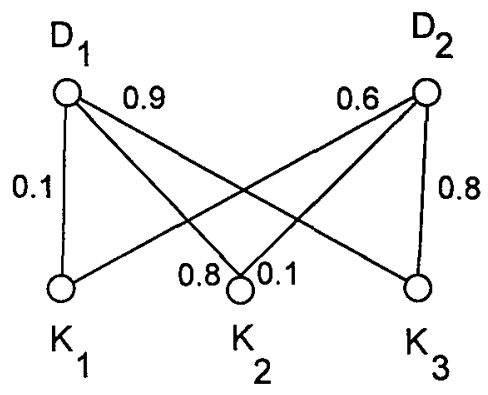

Figure 2.2: Simple neural network for two documents and three key terms.

For IR purposes each key term can be associated with an input neuron and each document with an output neuron. A query is presented to the network by activating the neurons which are associated with the desired key terms. The network then calculates the output signals. The activated output neurons correspond with the desired documents. Recall and precision can be improved by training the network. Training is an essential ingredient of neural networks. In principle, all weights initially are assigned random values. After the user has evaluated the results of a query, the system can adjust the weights according to a particular learning rule. Several learning rules are known, varying from very simple to complex ones.

Figure 2.2 shows a network with three input neurons, $K_{1}, K_{2}$ and $K_{3}$, representing the key terms gallstone, angina, and complication, and two output neurons, $D_{1}$ and $D_{2}$, representing two documents. The activation of an output neuron $D_{i}$ is a function of its total input. In this example it is defined as:

$$
\operatorname{activation}\left(D_{i}\right)=\operatorname{sgn}\left(\sum_{j} w_{i j} a_{j}-1\right)
$$

where $w_{i j}$ is the weight between nodes $i$ and $j, a_{j}$ is the activation of neuron $K_{j}$ and sgn is defined as:

$$
\operatorname{sgn}(x)=\left\{\begin{aligned}
-1 & \text { if } x<0 \\
0 & \text { if } x=0 \\
1 & \text { if } x>0
\end{aligned}\right.
$$

A document is selected if and only if the activation of the corresponding output neuron is greater than zero. If a query activates the neurons of gallstone $\left(K_{1}\right)$ and complication $\left(K_{3}\right)$ then the activations of the output neurons $D_{1}$ and $D_{2}$ are:

$$
\begin{aligned}
& \operatorname{activation}\left(D_{1}\right)=\operatorname{sgn}(1 \cdot 0.1+0 \cdot 0.8+1 \cdot 0.9-1)=\operatorname{sgn}(0)=0 \\
& \operatorname{activation}\left(D_{2}\right)=\operatorname{sgn}(1 \cdot 0.6+0 \cdot 0.1+1 \cdot 0.8-1)=\operatorname{sgn}(0.4)=1
\end{aligned}
$$


Output neuron $D_{2}$ is activated because its value is greater than zero; therefore document 2 is retrieved. If both activations were greater than zero, both documents would have been retrieved.

The system can learn by taking into account relevance feedback. Normally, relevance feedback is used to reformulate a query, but in the connectionistic model it is also used for the improvement of the document representation. In the example of Figure 2.2 the user may decide that $D_{2}$ is not relevant. In that case the weights between $D_{2}$ and the activated input neurons, $K_{1}$ and $K_{3}$, are decreased. Weights are increased for documents that are judged relevant. The initial weights can be determined by taking random weights and training the network on a set of documents with known relevances. Another approach is to determine the weights in a way similar to vector model indexing.

It is striking that a network with only an input layer and an output layer strongly resembles the vector model. This resemblance disappears when hidden layers or connections between input neurons are added. Layaida and Caron (1994) do the latter: a connection between two input neurons is made if the corresponding key terms occur in at least one document. Thus, neurons of synonymous key terms are also activated.

Advantages of the connectionistic model are:

- Learning is a part of the model.

- Hidden layers make the constraint of independent key terms superfluous.

No results of large-scale projects are available yet. This may be due to the number of neurons required to represent large numbers of documents and key terms and the resulting number of computations, specially in the learning phase. Crouch et al. (1989), Rose and Belew (1991), Chen et al. (1993), and Layaida and Caron (1994) use this model.

\subsection{The Twelve Characteristics}

By choosing an IR model the IR system is not completely determined; the IR model is only a basis. An IR-system designer has to decide on many characteristics whether they should be included, and how they should be realised. This section discusses twelve characteristics of the systems we examined.

Table 2.1 summarizes the characteristics (vertical) of the examined systems (horizontal). The model row indicates whether the Boolean, vector, probabilistic model, Bayesian, or connectionistic IR model is used. For the other characteristics (search intermediary, discussed in Subsection 2.3.1; domain knowledge, Subsection 2.3.2; relevance feedback, Subsection 2.3.3; natural-language interface, Subsection 2.3.4; graphical query language, Subsection 2.3.5; conceptual queries, Subsection 2.3.6; 


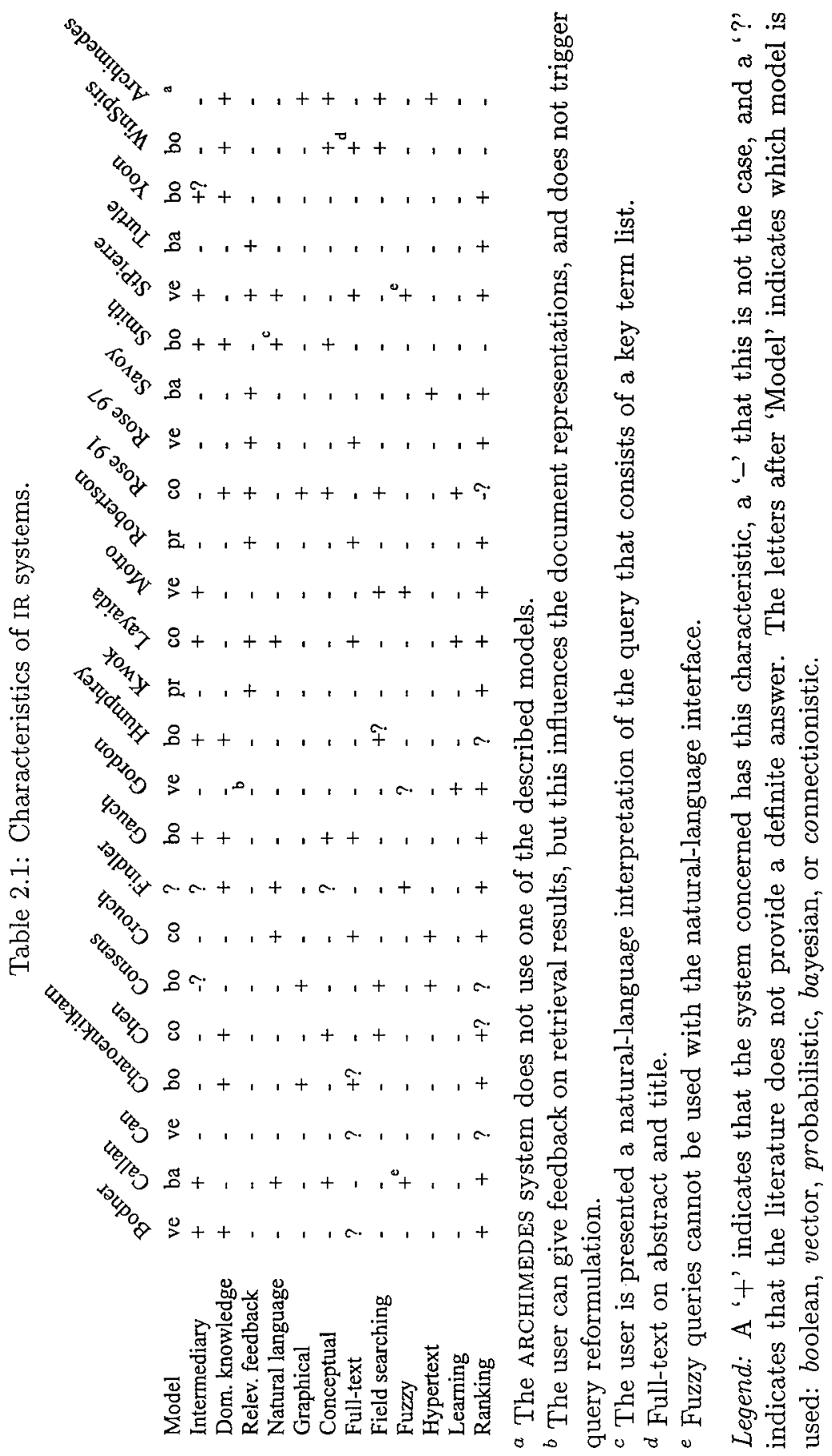


full-text IR, Subsection 2.3.7; field searching, Subsection 2.3.8; fuzzy queries, Subsection 2.3.9; hypertext integration, Subsection 2.3.10; machine learning, Subsection 2.3.11; and ranked output, Subsection 2.3.12) it is indicated whether they are used in the system. Every system is denoted by the first author of the reference used. In the last column the characteristics required for ANS are indicated. These are further discussed in Subsection 2.5.2.

\subsubsection{Search Intermediaries}

The more powerful the query language of an IR system, the more accurate the user can formulate the query. However, formulating a query becomes more difficult for the users. It is therefore not surprising that especially Boolean IR systems aid users with the formulation and reformulation of queries. Such an aid, which acts as an intermediary between user and IR system, is called a search intermediary.

There are several reasons for providing an IR system with a search intermediary. We already mentioned hiding a complex query language. Another purpose is providing active help during formulation and reformulation. Finally, a search intermediary can be used to limit the cost of searching if connection time is charged. The user formulates the query off-line with the search intermediary and only then connects to the IR mechanism. The system of St. Pierre et al. (1993) makes extensive use of this approach; it is even possible to make the search intermediary connect to multiple IR mechanisms for one query.

An intelligent search intermediary is a search intermediary that helps the user with the aid of knowledge of the domain of the documents and/or search tactics. An obvious way to realise an intelligent search intermediary is through an expert system. The user formulates a problem (how do I find exactly all the documents I need) and a model of the knowledge of a human expert (e.g., a librarian) is used to solve the problem.

As a case in point we refer to the Automatic Query Reformulation system of Gauch and Smith (1991). The underlying system contains the complete contents of the documents, and can retrieve relevant text fragments given a Boolean query. A search intermediary acts as an interface between this system and the user. It consists of a domain knowledge representation and an expert system that can reformulate queries and present retrieval results in order of estimated relevance to the user. Relevance of a text is judged on the basis of the relative frequency of the query key terms in the text, whether the key terms were proposed by the user or the system, and the contextual distance between the key terms. At the reformulation stage different techniques are applied:

- Expansion of queries by adding related terms

- Adaptation of the context (e.g., how close two key terms must be in the text)

- Manipulation of Boolean operators (e.g., replacing an AND by an OR) 
We have looked at nine systems with a search intermediary: Bodner and Song (1996), Motro (1988), Smith et al. (1989), Gauch and Smith (1991), Callan et al. (1992), Humphrey (1992), Yoon et al. (1992), St. Pierre et al. (1993), and Layaida and Caron (1994).

In the four subsections that follow, we discuss characteristics of IR systems that are often realised through a search intermediary: domain knowledge, relevance feedback, natural-language interface, and graphical query language.

\subsubsection{Domain Knowledge}

Knowledge of the domain of the documents may be used in the IR process. This may be especially useful when reformulating a query; the query may be broadened (or narrowed) by replacing a key term by a more general (or a more specific) key term. According to Smeaton and Rijsbergen (1983) reformulation should be done based on domain knowledge instead of statistical deliberations. Addition of key terms to a query on the basis of statistical relations with terms in the query may have negative consequences for the results. For instance: adding the key term connectionism to a query containing the key term neural may be done as a consequence of the cooccurrence of both key terms in many documents. As an undesired result also documents about neural disorders will be found.

Domain knowledge can be represented in several ways. One way is by a thesaurus; a list of terms that states for each term its synonyms, more specific terms, and a more general term (ISO, 1986). If a query with the key term choledochojejunostomy results in too few hits, the system may, after consulting the thesaurus, replace choledochojejunostomy by the more general term biliary tract surgery.

An example of a large thesaurus (some 70,000 concepts with some 96,000 key terms) containing general knowledge is WordNet (Miller, 1990; Miller, 1995). It is used by Bodner and Song (1996) and Charoenkitkarn et al. (1995). Bodner and Song use two thesauri to expand queries automatically: a domain-specific thesaurus and WordNet. Their experiments showed improved effectiveness. Charoenkitkarn et $a l$. only let the user browse the thesaurus. This facility was not very useful because the concepts in WordNet were too general. Voorhees (1993) also reports negative results with the use of WordNet: she disambiguated queries and key terms by using the is-a relations in WordNet, but it had a negative effect on the effectiveness of the system. Krovetz and Kroft (1992) provide an overview of the subject of ambiguity in IR.

The construction of a thesaurus is a difficult and tedious task; it requires a human domain expert. Automatic construction has been investigated by various researchers (e.g., Crouch and Yang, 1992; Chen et al., 1993; Jing and Croft, 1994; Bodner and Song, 1996; Chen et al., 1996). The resulting thesauri are not as detailed and accurate as manually constructed thesauri because these approaches are all based on statistical methods. It is not possible to determine automatically the exact nature 
of the relationship between two key terms, only whether they are related (or at best, that one key term is narrower than the other).

Another but similar way of representing domain knowledge is by a semantic network. Semantic networks were originally developed to model the human representation of knowledge. Major components are concepts and relations between concepts. A relation between two concepts constitutes a fact. Example facts that can be represented are 'choledochojejunostomy is a kind of biliary tract surgery' and 'gallstone has complication cholecystitis'. A semantic network uses a broader range of relations than a thesaurus, hence a query mechanism that uses a semantic network can perform more sophisticated reasoning about the improvement of queries than a query mechanism that uses a thesaurus. For the medical domain a CD-ROM exists with a semantic network containing a large number of medical key terms (over 300,000) and their relationships. This Unified Medical Language System (UMLS) from the American National Library of Medicine (Lindberg et al., 1993a) also encompasses the well-known Medical Subject Headings thesaurus (MesH) which is used in many medical IR systems (Lowe and Barnett, 1994).

WinSpirs, Chen et al. (1993), and Charoenkitkarn et al. (1995) make passive use of domain knowledge: the system supplies it to the user. Active use of domain knowledge, that is, the system itself uses the domain knowledge during query formulation, is made by Smith et al. (1989), Gauch and Smith (1991), Rose and Belew (1991), Findler et al. (1992), Humphrey (1992), Yoon et al. (1992), and Bodner and Song (1996).

\subsubsection{Relevance Feedback}

A system that can reformulate a query on the basis of the user's evaluation of query results makes use of relevance feedback. The idea is that the new query will retrieve more documents like those that were relevant (according to the user) and less documents that are like those that were irrelevant. In the vector model this can be realised simply by assigning a higher value to key terms from the query that occur in relevant documents, and to those that occur in irrelevant documents a lower value. This works best when relevant documents are tightly clustered (in terms of key-term space), irrelevant documents are also tightly clustered, and the distance between relevant and irrelevant documents is relatively large (Salton and McGill, 1983). Techniques have been devised to realise relevance feedback in the Boolean model, but they are not commonly applied. In general, relevance feedback does improve retrieval effectiveness greatly (Salton and Buckley, 1990). A limitation is the dependence of the user's willingness to supply feedback.

Relevance feedback is applied by: Kwok (1990), Rose and Belew (1991), Savoy and Desbois (1991), Turtle and Croft (1991), St. Pierre et al. (1993), Layaida and Caron (1994), Robertson et al. (1996), and Rose and Stevens (1997). 


\subsubsection{Natural-Language Interface}

A reason for unsatisfactory performance may be the language used to formulate queries: a formal language is an obstacle for many people. A natural-language interface enables the user to formulate queries in a natural language. For instance:

I am looking for scientific publications on complications that may occur with choledochojejunostomy.

There are two approaches to dealing with such a query: one can either parse the query and try to 'understand' its meaning, or one can try to sift out the important key terms. The former approach is realised as a special kind of search intermediary because it translates a natural-language query to a query in the language of the underlying IR model.

Turtle (1994) compared the effectiveness of natural-language queries (on a number of systems) with Boolean queries (on one system). Recall and precision were clearly higher for the natural-language queries than for the Boolean queries. There are, however, some disadvantages:

Automatic processing Understanding natural language is still a difficult task for computers. Within a specific domain, i.e., a well-defined limited domain, the results are nowadays satisfactory. This disadvantage does not pertain to the approach where key terms are only sifted from the query.

Ambiguity When using natural language there is a greater chance that a query is ambiguous. This holds especially for short queries. If the system identifies an ambiguity, it can ask the user which of the possible meanings is intended.

Laboriousness Formulating and typing entire sentences as queries takes more time than entering a few key terms.

Crouch et al. (1989), Callan et al. (1992), Findler et al. (1992), St. Pierre et al. (1993), and Layaida and Caron (1994) allow their users to formulate their queries in natural language. From these four only Crouch et al. (1989) really try to parse the sentences; the other systems simply eliminate uninteresting words and pass the remainder to the query mechanism. EP-X (Smith et al., 1989) has a completely different approach: it accepts a list of key terms as a query and then formulates possible interpretations of the query in natural language leaving the choice to the user.

\subsubsection{Graphical Query Language}

An alternative way of formulating queries is by manipulating graphical objects. This looks like a simple and intuitive means of describing an information need to an IR system. 


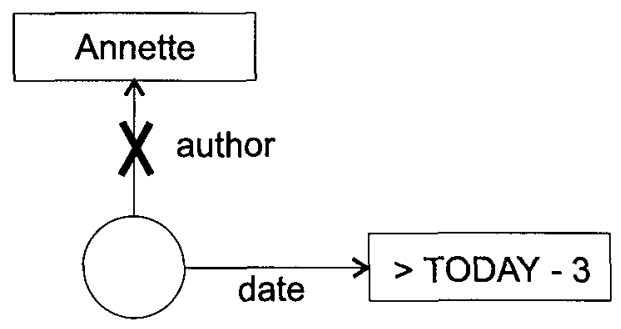

Figure 2.3: A query in GraphLog (drawn after Consens and Medezon, 1989).

The graphical query language GraphLog (Consens and Medezon, 1989) has a great expressive power. Figure 2.3 (drawn after Consens and Medezon) is an example of a query in which documents are requested that were created three days ago by others than the user (Annette). However, in our opinion this graphical query does not look all that simple.

The expressive power of the system described by Rose and Belew (1991) is limited and - therefore - easier to handle than a textual query language. The system is based on the connectionistic model. The formulation of a query does remind of 'query-by-example' as used in some database systems. The user selects some key terms or documents and the system shows in a graphical way documents that are similar to the selected ones. Since pairs of documents may be linked, indicating that a relationship exists between the two documents, these links are also shown. The user then indicates which of the retrieved documents look interesting and which do not. With this information the system can retrieve and show other documents, which in their turn can be evaluated so as to find more documents.

Charoenkitkarn et al. (1995) describe an even simpler graphical query language: queries are constructed by clicking on terms in the document shown on the screen. By drawing a line between two terms they are connected by the AND operator.

\subsubsection{Conceptual Queries}

Most of the IR systems do not impose any restriction on the user in choosing key terms for a query. This has a number of disadvantages (Sudarshan, 1979):

Synonym problem User and indexer may use different key terms for the same notion, causing too few documents to be retrieved.

Spelling problem User and indexer may spell a key term differently causing too few documents to be retrieved.

Homonym problem Using a key term which has multiple meanings will result in the retrieval of documents that concern all of these meanings, although the user is interested in only one. 
Other systems limit the user to a fixed set of key terms (i.e., fixed as long as the document set remains unchanged). Retrieval is not limited to the literal key word, but also comprises the associated synonyms and spelling variations. This type of query is called a conceptual query. Thus, with a conceptual query one does not search for terms but for concepts. Medical IR systems often use a large thesaurus with medical terms: the Medical Subject Headings (MesH) of the National Library of Medicine (Lowe and Barnett, 1994).

Systems that allow conceptual queries are: WinSpirs, Smith et al. (1989), Gauch and Smith (1991), Rose and Belew (1991), Callan et al. (1992), and Chen et al. (1993)

\subsubsection{Full-Text IR}

Traditional library systems can search for key terms that are - often manually - assigned to a document by an indexer or for key terms that occur in the title. Full-text IR systems search the entire document contents for a key term.

A document is represented by the words that occur in it; the indexing does not require human intervention. Because a full-text IR system has access to the entire text and not only to the key terms, these systems provide operators that select on proximity of key terms in the text, whether key terms are in the same sentence, or in the same section, chapter or document. Because the user is not limited to a fixed set of key terms full-text IR leads to a higher recall and, in general, to a lower precision.

Full-text IR systems are: WinSpirs ${ }^{3}$, Crouch et al. (1989), Kwok (1990), Gauch and Smith (1991), St. Pierre et al. (1993), Layaida and Caron (1994), Charoenkitkarn et al. (1995), Robertson et al. (1996), and Rose and Stevens (1997).

\subsubsection{Field Searching}

We may have raised the impression that queries always consist of key terms that are about the content of documents. However, a user may want to constrain the search to documents of a particular author. In some IR systems this is possible by supplying a key term that is a field. In these IR systems the documents are like records in a database. One of the fields is the key term field, which is the default field to be searched by a query. Other fields are, for instance, author, year, publication type, and journal. An example of a query with a field is: gall bladder surgery AND year = 1997. Or for our sample user, who wants to indicate that only scientific publications are to be retrieved: choledochojejunostomy AND type $=$ scientific publication. Since documents rarely state whether they are scientific or not, scientific publication cannot be supplied as ordinary key term.

\footnotetext{
${ }^{3}$ Only title and abstract, not the actual document content, are indexed full text.
} 
IR systems that allow field searching are: WinSpirs, Motro (1988), Consens and Medezon (1989), Rose and Belew (1991), Humphrey (1992), and Chen et al. (1993). Many systems that are actually used in practice include field searching. It is an obvious characteristic. Presumably it is not often used in experimental systems because it is well-understood and not interesting to experiment with.

\subsubsection{Fuzzy Queries}

Fuzzy queries or vague queries allow the user to indicate for each key term in the query how important it is relative to the other key terms in the query. This is very useful in the following case: suppose the query choledochojejunostomy results in too few documents and the query gall bladder surgery in too many. The query choledochojejunostomy AND gall bladder surgery will of course retrieve even fewer documents (or the same documents). But by indicating that the last key term is less important this can be alleviated: for instance

\section{choledochojejunostomy:0.9 OR gall bladder surgery:0.3}

A document must be about choledochojejunostomy for an important part and only to some extent about gall bladder surgery to be retrieved.

The importance of key terms in a query may be expressed absolutely, for instance by specifying a number between 0 and 1 for each key term, or relatively, by arranging the key terms in order of importance. Users find it easier to indicate relative importance than absolute importance (Lee, 1994).

The best model for fuzzy queries is the vector model because the relevance can easily be incorporated into the query vector. Our sample user from Section 2.2 will replace the ones and zeroes in the query

$$
\left(\begin{array}{l}
1 \\
0 \\
1
\end{array}\right) \text { by }\left(\begin{array}{c}
1 \\
0 \\
0.5
\end{array}\right)
$$

if the key term complication is less important. The Boolean model does not allow fuzzy queries, but several extensions of the Boolean model do. These are the fuzzy set model (Sachs, 1976), the P-Norm model (Salton et al., 1983), and the InfiniteOne model (Smith, 1990). Properties of these systems are discussed by Lee (1994).

Systems that allow fuzzy queries are described by: Motro (1988), Findler et al. (1992), Callan et al. (1992), and St. Pierre et al. (1993). In the latter two systems, fuzzy queries cannot be used in combination with the natural-language interface.

\subsubsection{Hypertext Integration}

Hypertext systems play an important role in documentary information systems. A hypertext system contains a large amount of information, usually text, divided into small parts called hypertext nodes. The user can travel from node to node by following links between the nodes. The user is supposed to find his information 


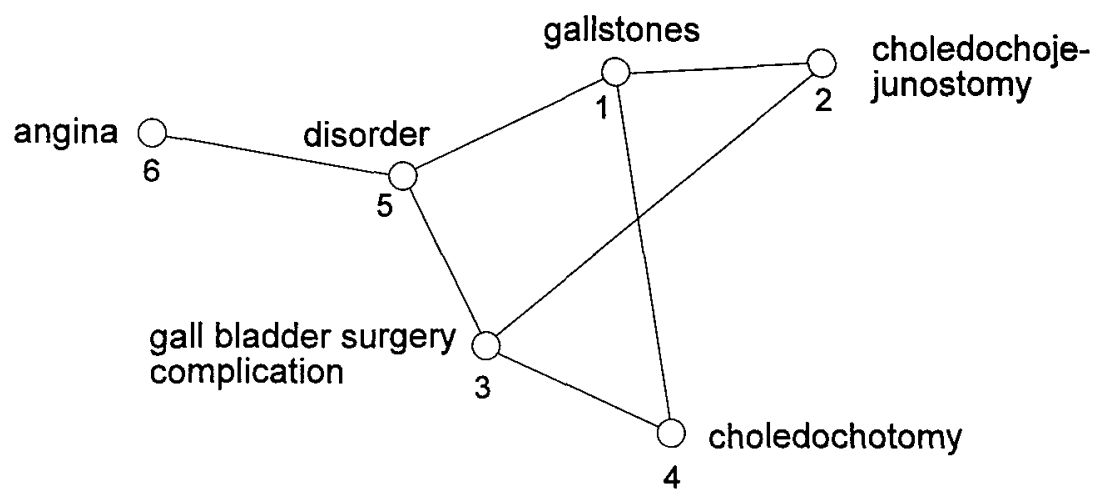

Figure 2.4: A hypertext network.

when he browses along. This uncontrolled way of searching for information can be augmented by IR techniques. The user can be brought to 'interesting places' from where he may start browsing.

Figure 2.4 depicts a hypertext network of a (fictitious) medical handbook. A query system that is integrated with the hypertext system will, after a query concerning complications with gall bladder surgery, show hypertext node 3 . It is up to the user whether he will look at adjacent nodes. The information in the nodes visited can be a reason for the user to formulate another query which may lead to another part of the hypertext network. Therefore, one can say IR systems and hypertext systems are complementary if they are integrated.

Consens and Medezon (1989), Crouch et al. (1989), and Savoy and Desbois (1991) describe such systems.

\subsubsection{Machine Learning}

An IR system learns if it improves its performance in some way as a consequence of former queries. The following aspects of an IR system are suitable for improvement by learning:

Document representation: The representation of a document may be improved if the user indicates that the document shown is or is not relevant.

Presentation: The order in which query results are presented to the user can be determined by taking into account the user's interests and preferences. For this a user model is required.

Thesaurus: On basis of co-occurrence of terms, a full-text IR system can conclude that terms are synonymous. 
There are various ways in which machine learning for an improved document representation is realised: Rose and Belew (1991) and Layaida and Caron (1994) use the connectionistic model, where - obviously - weights are changed on the basis of the user's rating for the query results.

Gordon (1988) has a completely different approach: he uses a genetic algorithm. Starting point is the observation that different users search for the same document in different ways. For each document various document representations are made: each document is represented by various document vectors. A query is matched with every representation of a document; the average of the matching scores determines whether a document will be retrieved or not. After the retrieval results have been displayed, the user indicates which documents are relevant and which are irrelevant. Now for each document it can be determined which representations fit the document best. The worst representations are discarded. New, hopefully better, representations are created by 'breeding' the best ones; that is, by concatenating randomly selected pieces of existing representations to a new representation.

The advantage of this technique lies in the increasingly better document representations that are obtained by adaptation to user preferences. Disadvantages are:

- The system must be used extensively in order to produce significantly better results.

- Storing multiple document representations takes extra disk space.

- Users may get confused when identical queries at different occasions yield different results.

\subsubsection{Ranked Output}

If a query results in a large number of retrieved documents, it is not feasible for the user to scan through the entire set. However, if the results are sorted in order of estimated relevance the user may safely limit himself to the top of the list. A system that sorts its results in such a way is said to produce ranked output (or: to apply relevance ranking).

In the evaluation of IR systems with ranked output, it is customary to determine the recall and precision at various cut-off points. For instance, recall and precision are determined for the 10,20, and 30 highest ranked documents. Another practice is to determine the recall at various precision value cut-offs (or vice versa). Thus, the ranking algorithm strongly influences the IR system's effectiveness.

Many IR systems provide ranked output. Harman (1992) provides an overview of existing ranking algorithms. She distinguishes two types: ranking of individual documents and ranking of sets of related documents.

We start discussing the first type. In the vector model, obviously, the similarity measure can be used as a basis for ranking. In the probabilistic model the document 
balloon angioplasty

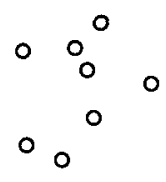

\section{choledochotomy \\ $\circ$ ○ం}

$0^{\circ}$ 。

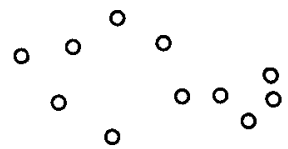

choledochojejunostomy

Figure 2.5: Clusters of documents concerning surgery.

relevance probability and in the connectionistic model the output neuron activation are used. In the Boolean model things are more complicated. The obvious measure is the number of matching key terms. This makes only sense when at least one OR operator is used. It gives hardly an ordering; it is more of a division into groups. Somewhat better is to take into account the inverse document frequency of each key term, which depends on the frequency a key term occurs in the entire document collection. This measure in fact ranks key terms. Charoenkitkarn et al. (1995) use for each matching document the within-document frequency (i.e., the number of times a key term occurs in a document) normalized by the document length. Another, hybrid, approach is to use the Boolean model to retrieve the documents and the vector model to rank them.

Rose and Stevens (1997) remark that the ranking should be consistent with the users' expectations. For instance, users expect a document with two matching key terms to be ranked higher than a document with only one matching key term, even if the key term frequencies indicate that the latter document is more important. Rose and Stevens found that their method of adjusting the similarity measure by taking into account the number of matching key terms did not have a negative influence on retrieval effectiveness.

Other systems that use the first type of ranking are described by: Gordon (1988), Motro (1988), Kwok (1990), Gauch and Smith (1991), Savoy and Desbois (1991), Callan et al. (1992), Yoon et al. (1992), Chen et al. (1993), St. Pierre et al. (1993), Turtle (1993), Layaida and Caron (1994), Bodner and Song (1996), and Robertson et al. (1996).

The best-known example of the second type of ranking is clustering. Generally speaking, this is arranging a number of objects in such a way that clusters emerge that group together objects that are similar according to some criterion. This principle is applied to IR in different ways. A possible criterion is the measure of similarity of documents as defined in the vector model. Documents that have many key terms in common form a cluster; different clusters denote different areas of interest. Fig- 
ure 2.5 shows documents that are clustered according to this criterion. All documents are assigned the key term surgery, but three different operations are concerned: balloon angioplasty, choledochotomy, and choledochojejunostomy. This results in three clusters. The balloon angioplasty cluster is somewhat distant from the other two because it is a different type of operation. In the system of Crouch et al. (1989) the result of a query is a hierarchy of clusters of possibly relevant documents, where documents that seem to be similar are in the same cluster. Can (1993) focusses on the problem of updating clusters dynamically, as determining the clusters anew every time documents are added can be very costly. Findler et al. (1992) use clustering in a different way: they divide the domain knowledge, which is represented in a semantic network, into clusters using the user's suggestions. Thus the search for documents can be limited.

\subsection{Information Retrieval and the World Wide Web}

The World Wide Web (Www, or Web) is by its diversity and dimensions a challenge for IR systems. First we explain what the Web is and why IR is important for it, then we discuss several IR systems that are used for the Web.

\subsubsection{The Word Wide Web}

The Web is a distributed client/server hypermedia system on the Internet (BernersLee et al., 1992). A hypermedia system is a hypertext system that contains multimedial information, that is, a combination of text, images, sounds, and movies. It is a client/server system: the hypermedia information resides on the server side and the user needs a client program to view the information. There are tens of thousands of Web servers world-wide, all connected by hyperlinks. That is why the Web is a distributed system. No central authority exists to coordinate the servers, or even register them. This makes it difficult for users to locate the information they need.

In the beginning of the Web, in 1990-1992, it was possible to browse through all information on all servers. When this was not possible anymore, subject directories started to emerge. In its simplest form, a subject directory is a list of Web sites that are important to a particular subject. Every entry has a hyperlink that takes the reader directly to the corresponding site. More sophisticated subject directories give short descriptions of the sites and break down the list into categories. The subject directories were never complete because they were compiled manually and the Web grew exponentially. The problem was tackled with the introduction of Web robots (or Spiders). They are computer programs that can automatically traverse the Web by recursively following all hyperlinks starting from one arbitrary server. The information gathered can be indexed and serves as the basis for an IR system. Examples of Web robots are Harvest (Bowman et al., 1994) and Momspider (Fielding, 1994). The first search engine - as a Web IR system is called - that was really usable 
because it covered a substantial part of the Web was Lycos. Other search engines followed. All Web search engines have in common that they use a Web robot to index text documents on the Web, ignoring non-textual information. Nowadays, a Web without search engines would be unthinkable. Despite of the maintenance problem, subject directories continue to exist. Some of them are still maintained by hand, others use Web robots to gather information and humans for classification. The differences between search engines and subject directories have grown smaller: some search engines also offer subject directories, and many subject directories now have a search facility.

\subsubsection{Search Engines}

We now discuss several Web search engines in terms of the IR system characteristics that were introduced in Section 2.3.

For the search engines discussed here it is not clear which IR model is used. They are commercial products, and it is therefore difficult to obtain information about their inner workings. The query language suggests that all use some kind of extended Boolean model. All have both a simple and an advanced user interface, allowing simple key-term searches and Boolean queries, respectively. The simple query interface may be viewed as a intermediary to the complex query interface. The systems do not provide for conceptual queries and do not have machine-learning capabilities. All engines provide ranked output.

AltaVista ${ }^{4}$ is a full-text IR system that also allows field searching. For instance, one can search in a title or a hyperlink. AltaVista can dynamically generate a graphical thesaurus, called LiveTopics (Bourdoncle, 1997), based on a query and its results. LiveTopics determines related terms by using statistics on co-occurrence of terms in the retrieved documents. The related key terms are grouped in a topic; related topics are depicted with a link between them. The user can click on key terms and thus include or exclude them in the query. For instance, the query gallstone resulted in 886 documents. Topics that were extracted included: gallstones, complications, abdominal, liver. The first topic contained the key terms gallstones, cholecystectomy, laporoscopic, surgery, surgical, abdomen (which is also a topic, surprisingly), incision, incisions. This facility works well when many documents are retrieved. For smaller numbers the resulting topic graph can be rather odd.

The Excite ${ }^{5}$ search engine is a full-text IR system that not only takes into account the key terms in the query, but automatically broadens the search to the synonyms of the key terms. The synonyms are automatically extracted from the documents. ${ }^{6}$ The Excite engine shows together with the query results a list of terms that are related with the query key terms. For instance, the query gallstone resulted in 554 documents. Related key terms shown were: gallstones, gall bladder, bile, pancreatitis,

\footnotetext{
${ }^{4}$ URL is: http://www.altavista.digital.com/

${ }^{5}$ URL is: http://www.excite.com/

${ }^{6}$ The mechanism is not clear.
} 
biliary, gastroenterol, thijs (this is the name of the author of an article about gallstones), laparoscopic, calorie, extracorporeal. The idea of this facility is similar to AltaVista's topic graph, but it is more limited. Another facility is relevance feedback: when the user has identified an interesting document, similar documents can be retrieved.

HotBot $^{7}$ is a full-text IR system that has extensive field search options. For instance: date, location, media type.

Infoseek ${ }^{8}$ accepts, apart from Boolean queries, also natural-language queries. Field searches are also possible. Like AltaVista and Excite, query results are accompanied with related topics; these can be selected to narrow the query. For instance, the query gallstone resulted in 2344 documents. Related topics shown were: dieting, medical specialties, gastroenterology. By selecting a topic the original query is narrowed to documents concerning the selected topic. As opposed to AltaVista and Excite, these topics are not determined dynamically, but are selected from a subject directory part of Infoseek.

Ly $\cos ^{9}$ offers the user various interfaces. There is a Boolean search interface with many operators, a natural language interface and Lycos Pro. With Lycos Pro it is possible for the user to influence the relevance ranking. For each factor taken into account during relevance ranking, the user can specify the importance that should be attached to it. Some factors are: frequency of words, words appear early in the text, words appear close together.

Yahoo $^{10}$ is actually a subject directory, but is also has a query interface. It differs from most subject directories in that categories are hierarchically organized. The user can either browse through the hierarchy of categories or compose a query. Because Yahoo is updated manually, searches typically have a high precision and low recall. Low recall is not necessarily a problem, because often the most important documents for a subject are present. For instance, the query gallstone resulted in two documents. Their titles were shown together with their place in the Yahoo hierarchy: Health: Diseases and Conditions: Digestion and Nutrition Disorders and Regional: U.S. States: New York: Cities: New York City: Business: Health: Providers: Clinics and Practices. Both documents were indeed very relevant to the query, yet only to laymen. If a query returns no documents at all, the query is automatically passed on to AltaVista.

It is not clear how many documents the search engines have indexed. They provide different figures and use different methods of counting them. However, it is safe to say that the engines discussed here contain some tens of millions of documents. The exception is Yahoo, which contains some hundreds of thousands of documents. Because of the importance of search engines for the Web, they are very frequently used. It is therefore not surprising that these search engines require considerable

\footnotetext{
${ }^{7}$ URL is: http://www.hotbot.com/

${ }^{8}$ URL is: http://www.infoseek.com/

${ }^{9}$ URL is: http://www.lycos.com/

${ }^{10}$ URL is: http://www.yahoo.com/
} 
computing power. For instance, AltaVista uses a machine with 10 processors, $6 \mathrm{~GB}$ memory, 210 GB hard disk to run its search engine on, a 1.5 GB memory, $30 \mathrm{~GB}$ hard disk machine to run its Web robot, and four other machines for handling Web traffic, indexing, and Usenet. ${ }^{11}$

Chu and Rosenthal (1996) propose evaluation criteria for search engines. These include: indexing (coverage, update frequency, portion of document that is indexed), search capabilities (a subset of the characteristics in Section 2.3), retrieval performance (recall, precision, and response time), output (options and content), and user effort (documentation and user interface). They compared AltaVista, Excite, and Lycos. No differences in the response times were found. AltaVista scored the highest precision, considering the ten highest ranked documents only. Recall was not determined. Notess (1997) discusses Yahoo and other subject directories. He concludes that despite of their incompleteness and inaccuracy, subject directories are effective for searching specific information on the Web.

\subsection{Chapter Conclusions}

Subsection 2.5.1 discusses general conclusions about current IR systems, Subsection 2.5.2 concludes which properties are important for the ARCHIMEDES system.

\subsubsection{Conclusions on Current IR Systems}

The construction of an IR system requires a number of decisions to be made. The most important ones have been discussed here, and where possible advantages and disadvantages were given. However, it is not possible to name the 'best choice' because it depends on factors as type and size of the application domain, type of users, and so on.

There are many techniques in IR but only a few have been used in large-scale systems: only the Boolean model is widely commercially available. The vector model too is used in commercial systems, but on a small scale. Both the Boolean model and vector model have proven themselves with large document bases, but other retrieval models have only been tested on small document bases; the connectionistic model and the Bayesian network model are possibly too complex.

New developments in IR are not to be expected so much from novel IR models but rather from characteristics as discussed in Section 2.3. Especially in the field of search intermediaries much research is being carried out. The Web is a challenging testbed for IR systems because of its size, diversity, and popularity.

\footnotetext{
${ }^{11}$ Usenet is an Internet service that is strictly not part of the Web, but is indexed by AltaVista and several other search engines. It can be viewed as a collection of thousands of electronic notice boards, all dedicated to different subjects, free for everyone to read and contribute to.
} 


\subsubsection{Requirements for the Archimedes IR System}

From the inventory above we have selected several needs and ideas. The following requirements for the ARCHIMEDES IR system are imposed:

- It should match with the facilities offered by the Information Network for knowledge representation such as concepts, link types, and document types (Subsections 2.3.2 and 2.3.8).

- There must be a good mixture of browsing and querying; browser and query mechanism must complement each other (Subsection 2.3.10).

- The system should be able to process some tens of thousands of documents. Not only should it store and represent this number of documents (moreover, this is not a part of the IR system, but part of the Information Network), it also should process queries within a reasonable time (Subsection 2.5.1).

On the basis of these requirements as well as the advantages and disadvantages mentioned we have determined which characteristics of IR systems are imposed for our system. Our choices are depicted in the last column of Table 2.1 on page 25. 


\title{
Chapter 3
}

\section{Design of the Archimedes Network System}

\begin{abstract}
This chapter is a slightly adapted version of: Dietz, J.L.G., Van der Pol, R., and Wiesman, F. (1997). The ARCHIMEDES Network System: a system for searching and accessing information in multiple multimedia sources. Journal of Intelligent Information Systems, Vol. 8, No. 1, pp. 77-101.
\end{abstract}

In Section 1.4 the Information Network was proposed as the underlying structure of the ARChimedes system. The name Archimedes Network System, abbreviated to ANS, refers to this Information Network. The Information Network and requirements concerning ANS are described in a paper by Hage et al. (1993). This chapter reports on the elaboration of a number of these ideas, particularly on a definition of the notion of document, and on document properties. Next to that, the functional architecture of an operational ANS implementation is provided. The problems of search and access, as identified in Section 1.4, are the main topics addressed by this chapter.

The outline of this chapter is as follows. Section 3.1 analyses the objects of search and access: documents and their properties. In Section 3.2 our approach to the search and access problems is presented, and an overview is given of the functional architecture of ANS. The two main components of this architecture are dealt with in the subsequent sections. In Section 3.3 we elaborate on the Information Network. It will turn out that this network can conceptually be viewed as an ordinary database after extending the database schema with a special kind of entity type: the document type. In Section 3.4 the third component, called the server, is discussed. The emphasis is on the browsing facilities offered by the server. Section 3.5 describes the results of an initial experiment. Section 3.6 concludes the chapter by summarizing the first research results and previewing the next steps towards the further realisation of ANS. 


\subsection{The Information to Be Searched and Accessed}

Before a software system can be specified that tackles the search and access problems, as introduced in Section 1.4, we must understand the major characteristics of the information to be searched and accessed. As a first step to such understanding, we consider all information that is searchable and accessible to be contained in documents. In Subsection 3.1.1 we define the notion of document and elaborate on two particular types of documents. Some properties of documents, relevant for search and access, are discussed in Subsection 3.1.2.

\subsubsection{Document Types}

Below we provide a definition of document. We discuss particular types of documents and how to access them with Document Access Programs (DAPS). Finally, we introduce hyperdocuments.

\section{Definition of Document}

The central notion in ANS is the type of thing one searches for and to which one seeks to get access. In accordance with common practice, we will call these things 'documents'. At the same time however we will strive for a definition of the notion of document that combines genericness with preciseness. Such a definition is built up and refined hereafter.

To start with, we define a document as an amount of stored information. This corresponds to everybody's intuitive notion: a document is or contains information, and this information is somehow captured in material substance. It also corresponds to the definition of document as, for instance, found in Webster's New Collegiate Dictionary (1975):

(a) a writing conveying information, (b) a material substance (as a coin or stone) having on it a representation of the thoughts of men by means of some conventional mark or symbol.

According to Webster's definition, a document must possess permanence. Transient information, for instance, a sequence of sound waves, is not considered a document. Our provisional definition of document contains the necessary condition for something to be called a document, namely that it is stored information. However, this condition is not a sufficient condition, since not every amount of stored information constitutes a document.

For the formulation of a necessary and sufficient condition, we would like to pinpoint the condition of permanence with a feature that can be verified by observation. Unfortunately, such a feature seems not to exist, which implies that it cannot be determined by observation whether something is a document. Therefore, we formulate a condition, that stems from the proviso that a document is a product: a 
document is the outcome of a production process performed by a person or under his responsibility; the person is called its producer (often, this is the author). The person creates a document by declaring an amount of stored information with some permanence to be a document. We therefore call stored information a document if its producer declares it to be so.

The above definition is broad; it includes both printed documents and documents stored in another manner. ANs is limited to searching and accessing a narrower class of documents, namely, those that are accessible by digital electronic computers.

In his historical overview of the definition of 'document' Buckland (1997) discusses even broader definitions; for instance, Otlet (1934) stated that documents can be three-dimensional. Briet (1951) defined a document as "any physical or symbolic sign, preserved or recorded, intended to represent, to reconstruct, or to demonstrate a physical or conceptual phenomenon." Consequently, Briet regarded an animal in a zoo, an object of study, as a document.

\section{Document Access Programs}

A particular type of document is the Document Access Program (DAP). The name already indicates its function: a DAP serves to access other documents (which are typically not DAPS). Hence, it is a computer program. But it is also an amount of stored information and in that sense it should be regarded as a document. This generic view on documents enables us to consider all information in the information sources to be contained in documents.

So, every document is accessed by means of a DAP. Mostly the DAP through which access to a document is obtained, is the same program as with which it was created. Examples of DAPs are MS Word, CorelDraw!, and WordPad. The emerging standardization of formats allows one to access documents by means of one of several DAPs, thus not necessarily the one with which it was created. At present this holds especially for graphical documents.

Accessing a document includes editing it, and presenting it. By presenting a document we mean: to make the text parts and image parts of the document visible on the video screen, to make the audio parts of the document audible, and to make both visible and audible the movie parts of the document. When presenting the document, the DAP controls the output devices, via the operating system. As a result, the document content is transformed into the corresponding perceptible form. It depends on the DAP and the hardware how 'faithful' the transformation is performed, that is, to what degree the transformation is information preserving. For instance, a monochrome video display may show a drawing in a document as having a single grey value, where in the stored document two different colours were present in the drawing. By editing a document we mean changing its storage representation, and therewith its presentation form. The latter two notions are discussed in Subsection 3.1.2. 


\section{Hyperdocuments}

Some of the documents present in the external sources may be created as hyperdocuments, that is, as compositions of (parts of) documents. We first elaborate on a definition of hyperdocuments and then discuss how these documents can be searched and accessed.

Several definitions are proposed for hyperdocuments. See, for instance, Campbell and Goodman (1988), Furuta and Stotts (1990), Halasz and Schwartz (1990), Garzotto et al. (1991), De Bra et al. (1992), and Haan et al. (1992). Although these definitions have a great deal in common, they are not identical. The definition we adopt is the one proposed by Halasz and Schwartz; it is named 'Dexter hypertext reference model'. The reason for choosing the Dexter model is that it allows for a smooth correspondence between our notion of document and the notion of atom as used in the model. It should be noted that we consider some of the other models also suitable for adoption.

According to the Dexter model a hyperdocument is a collection of atoms, anchors, links, and attributes. An atom may be anything that has some information content. An anchor is defined as a location or an item within the content of an atom. A link is a connection between two anchors. An attribute is information about an atom or about a link.

To exemplify the notion of hyperdocument, we consider an encyclopaedia. When an encyclopaedia is transformed into a digitally stored hyperdocument, the text of each subject treated typically forms the content of one atom, and so does each picture that illustrates the text. Whereas printed encyclopaedias by their nature allow for text and pictures only, digitally stored encyclopaedias allow for audio fragments and video fragments as illustrations too. It is common practice to choose one atom for each audio or video fragment and to represent the relationships among the contents of the atoms by links, with anchors serving as an aid. Imagine, for instance, a short video fragment of Kasparov, serving to illustrate a textual exposition on this chess world champion. The video fragment and the text are each stored in a separate atom. Further, an anchor is defined as the word Kasparov within the atom that contains the text. Another anchor is defined as the entire video fragment in the other atom. Then, a link is defined between the two anchors. In general, links and their accompanying anchors constitute the formal counterparts of relationships among the contents of atoms.

As an atom is either an entire document or a part of a document, links between atoms in a hyperdocument either connect parts of a single document or parts of different documents. They are called intra-document links and inter-document links, respectively (Glushko, 1989).

As a case in point, an intra-document link may connect a noun phrase in the text of a document to an illustration located elsewhere in the same document. This is similar to the encyclopaedia example above. Inter-document links, for instance, allow for the composition of a hyperdocument out of documents originally created 
as individual documents. The distinction between intra-document links and interdocument links plays a part in Section 3.4.

Note that, as each document may be a hyperdocument, several levels of hyperdocuments may be built up. Such structures are beyond the scope of this thesis, however. For a discussion of complex, multi-layered hyperdocuments we refer to the original definition of the Dexter model (Halasz and Schwartz, 1990) and to the definition of the Tower Model (De Bra et al., 1992).

The possibilities to search a hyperdocument are somewhat more varied than to search other documents. Linear documents are searchable with the aid of content descriptions. For hyperdocuments the same holds, not only for the document as a whole, but also for individual atoms if these are provided with content descriptions. A further possibility for search is offered by the presence of links in hyperdocuments: links allow for search via the relationships between the contents in atoms of hyperdocuments.

Access to a hyperdocument is obtained, like access to all documents, by means of a DAP. The DAP of a hyperdocument presents the contents of the atoms of which a document is composed, in the first place. In addition, if a presented atom has a link to another atom, the DAP presents the other atom on the user's request. This facility allows one to 'read' the document in a non-linear fashion, as described by, among others, Nielsen (1990b). Some DAPs also offer a command to follow a link backward, that is, to access the atom that refers to the selected atom.

\subsubsection{Document Properties}

The design of ANS highly depends on the properties of the documents. The properties are discussed in this subsection, starting by representation types. We then focus on a particular representation type: the presentation. Part of the presentation is the 'medium kind'. We introduce a new medium kind, 'formula'. Finally, we discuss how documents can be distinguished.

\section{Representation Types}

We distinguish three representation types: (1) for storing the document, (2) for handling the document, and (3) for presenting the document.

Our definition requires that the information in a document is stored. This is only possible if there exists a storage substance, containing a permanent representation of the document. We call this type the storage representation of the document. It satisfies a convention, called format. The format prescribes the organization of the information (encoded in bits). A document containing the information is stored in files. Each copy of such a document is stored according to exactly one format, mostly a standardised format. ${ }^{1}$

\footnotetext{
${ }^{1}$ Examples of standardised formats for the storage of video clips are MPEG, Motion JPEG, Quicktime, and AVI format.
} 
The transformation of a storage representation into a presentation is performed by a document presentation program. The storage representation and the presentation form of documents are important for distinguishing documents, hence for search of documents. Below, we discuss the presentation form. The end of the subsection discusses how to distinguish documents.

The second representation type plays a part in the handling of documents. As the storage representation, the representation for handling is not observable. The format is usually identical to that of the storage representation. In contrast to the storage representation, a handling representation typically has a temporary nature: it is created for handling, and is discarded once the handling has been finished.

The third representation type is for presenting a document to a human being. Since the storage and handling representations of a digital document typically are not observable by the human senses, a different representation needs to be made, meanwhile preserving the information. As opposed to the storage representation, the presentation representation (presentation form) usually has a temporary nature: a specific presentation of a document is created when a person wants to inspect the document, and is discarded when the person has finished the inspection.

\section{Presentation Form}

The presentation form is, among other things, characterized by the kinds of medium used. Medium kinds differ in their relation to the required physical senses for observation, and the sign types used in the presentation. For the scope of the ARCHIMEDES project, we distinguish the known medium kinds text, audio, video, smell, and tactile (as mentioned in, e.g., Iso/rEC, 1993). Moreover, we introduce a new medium kind, formula.

Information of the kind formula consists of expressions in a formal language. The addition of the kind formula makes it possible to distinguish information that can be processed automatically at a syntactic level from information where automatic processing can only fully exist at the lexical level (cf. any text processor). Moreover it offers the possibility to consider databases to be just documents, while being distinct from other documents. Below, we elaborate on the medium kind formula.

The medium kinds of a document impose requirements on the storage representation, on the carrier of the document, and on the computer system that stores and presents the document. For instance, a larger storage space is required for an image of a certain size than for a text of the same size (even if the image shows a text only).

As already indicated, the presentation form concerns more than the medium kind. For instance, in text documents the presentation also concerns the lay-out of the text, the font type, the font size, and so on; in documents of the kind audio it concerns the sound volume. In a book, the presentation is also used to indicate the division into parts, like chapters, sections, and a table of contents. When a 
book is presented, the user recognizes its parts mainly through the presentation: the headings of the parts have lay-outs that differ from the standard text lay-out.

Since presentation of a document occurs through a DAP the DAP must have information on the required presentation of the contents of the document. The information the DAP needs is contained in the storage representation. The medium kinds, the font and font size are encoded in the document file(s) as symbols; in some cases these symbols are elements of an explicitly defined mark-up language. A number of standard mark-up languages is defined, for instance, SGML (Smith, 1988) and ODA (ISO/IEC, 1989).

\section{The Medium Kind 'Formula'}

In the introduction of presentation forms we stated that documents of the medium kind formula consist of expressions in a formal language. These expressions have a well-defined (formal) syntax as opposed to expressions in a natural language.

A large subclass of documents of the medium kind formula is constituted by databases. In databases, either relational, network, or hierarchical, the internal semantics is explicitly defined in the document itself. Each such document has, as part of its content, a fact base (FB) that contains the stored data, a conceptual schema (CS) that contains the definition of the fact types allowed for in the FB, and a metaschema (MS) that contains the definition of the fact types allowed for in both the CS and the MS itself (for a detailed explanation, see Section 3.3). The DAP through which the FB is accessed makes use of the CS to access individual facts in the database (Date, 1990). The DAP of a database is usually called the DataBase Management System.

Knowledge bases are also documents of the kind formula. We regard a knowledge base as a document that has a knowledge-based system as DAP. This view corresponds to Date's (1990). There seems to be no consensus in the literature on the notions of knowledge-based system and knowledge base. Yet, we believe it safe to say that in general a knowledge base may be stored in a standardized database, like any document of the kind formula. The knowledge base then has an explicitly defined syntax (by the CS) and is accessed by its DAP via the database management system (which, for instance, ensures integrity of data). Until now however, many knowledge bases have a syntax that is only implicitly defined in the program code of the knowledge-based system and not explicitly defined in the document that contains the knowledge base. This may hold, for instance, for a knowledge base to be accessed by a PROLOG interpreter.

Although the content of a fact base (FB) and the conceptual schema (CS) of a database could be considered as distinct documents, they also have a strong interrelationship. This relationship is that any (version of a) FB is meaningless and thus useless without the appropriate version of the corresponding CS. A similar strong relationship exists between the CS and the metaschema (MS). For these reasons, the $\mathrm{FB}, \mathrm{CS}$, and MS may be considered as the content of one (composite) document. If a 
suitable DAP is available, they may be considered as three atoms in a hyperdocument.

\section{Distinguishing Documents}

Each property of a document may be used to distinguish it from other documents; if it differs with respect to some property, it is a different document. For each document a minimal number of properties is necessary to distinguish it from all other documents. So as to identify these properties, we regard each document to consist of document content and document descriptors. By content of a document we refer to what is in the document. The content of a document is determined by the presentation of the document. ${ }^{2}$ A document descriptor provides additional information to the document content, such as author and title. The distinction between contents and additional information is common practice with printed documents.

Further, we introduce document copy. As with printed documents, two documents that are identical in everything except the carrier (and possibly the moment of its recording on the carrier) are considered copies of each other.

Most documents can be distinguished by their contents. If this fails, they can be distinguished by their descriptors. If this fails again, additional descriptors are necessary. For instance, a book may be distinguished by its content, and descriptors containing title, author name, and date of publication. The title, author, and the date of publication are needed to distinguish between documents with identical contents. As such, new editions and reprints are recognized as different documents if they have different contents, and as identical if they have identical contents. A translation of a document yields a different lexical content (and usually slight changes in semantic content), and hence is a different document. Another example is: a photograph may be distinguished by content, photographer, and moment and location of recording.

Instead of the content itself, information derived thereof may be used. Index terms are an example of such derived information.

For documents with a content that is subject to change, it may be impractical to distinguish them by content. While the content changes, the user may want to conceive the changed document as the previous document. For instance, it seems not logical to consider different versions of a database (with different contents) to be distinct documents. The content of a database may change almost continuously. As these changes are often minor, the different versions would contain predominantly the same information. Its users want to conceive the different versions as one 'thing', speaking of, for instance, 'the personnel database', referring to a database with personnel data (names, addresses, etc.) that is updated weekly.

Obviously the content of a database is not suited for distinguishing database documents; neither is a time indication, for instance, the creation of a last change. Instead, an identifier must be assigned by the creator. As long as the creator does

\footnotetext{
${ }^{2}$ The semantic content of a document lies enclosed in its presentation form.
} 
not want to distinguish between databases with different contents or moment of last change, he assigns the same identifier. As soon as he wants to distinguish, for instance, after a major change, he creates a new document by assigning a new identifier to the database (the old database may remain as co-existent). This is done, for instance, after a change in the CS of a database, since such a change often yields a major change in the FB.

For documents distinguished by among other things their contents, we need an operational notion of content identity. Content is related to the presentation form of a document. We define the contents of two documents as identical if they have the same presentation form. A practical condition concerns the storage representation. If the representations of two digital documents are identical, assuming they are stored according to the same format, their contents are also identical.

If a document is to be searchable by ANs, it must be assigned a unique identifier on the basis of its distinguishing properties (i.e., contents and descriptors). ANS focusses on only one of the distinguishing properties: the content. The content is characterized by index terms.

\subsection{The ANS Architecture}

In this section, our approach to the search and access problems is presented. In Subsection 3.2.1, we describe the general approach. In Subsection 3.2.2 we describe an architecture for an implementation and discuss a first prototype.

\subsubsection{The ANS Approach to Search and Access}

Searching documents is typically done with Boolean queries. When such searching is performed without any support, it yields a poor effectiveness. Not all relevant documents are found, whereas irrelevant documents are found, or, in other words, the recall and the precision are below $100 \%$. This was shown by Blair and Maron (1985).

For improving the effectiveness of search, different approaches have been tried. We focus on approaches using information on the subject domain, such as a thesaurus. We call information on the subject domain a domain representation. Using a domain representation has the advantage that users with poor knowledge of the structure and terminology of the domain can be supported.

We approach the search problem with

- Meta-information, which consists of representations of

- The domain discussed in the documents

- The contents of the documents, by means of document descriptors (e.g., key terms, document type and medium kind) 
- Relations between documents (e.g., hyperlinks)

- A browse mechanism, which is a graphical user interface that presents the meta-information coherently on a video screen. It serves to offer initial domain knowledge to users not familiar with the domain and key terms for queries. We define metabrowsing as the act of searching through meta-information by browsing. Metabrowsing can be considered as an IR model (which was defined in Section 2.2); in the ANS browse mechanism documents are represented by links to document descriptors, queries are represented by key terms and filters (the latter will be discussed in Section 3.4), and the matching procedure is performed by the user.

- A query mechanism, which is a standard mechanism, that may be used after query terms are found with the browser.

With the combination of the fore-mentioned meta-information, browse mechanism, and query mechanism, we expect to improve the effectiveness and efficiency of searching relevant documents.

The idea of having links between nodes as well as the usage of several navigation techniques (Nielsen, 1990a) necessary for browsing through the Information Network came from the hypertext paradigm (see, e.g., Halasz and Schwartz, 1994). The usefulness of typed links is demonstrated by Baron et al. (1996). Some hypertext systems use a special navigation tool: they visualize the hypertext network (Halasz et al., 1987; Conklin and Begeman, 1988; Utting and Yankelovich, 1989; Lenman et al., 1996). This navigation tool is comparable to ANs' graphical interface. This is also the case for the interface of graphical IR systems. These are described bij Consens and Medezon (1989) and Rose and Belew (1991). They offer a graphical way of specifying a query, but not a graphical way of finding the right key terms. The UNAMED system (Hristovski et al., 1994) contains a graphical browser. It is not a general IR system but is specially designed for the medical domain. Because it only shows the direct neighbours of a concept, it provides a limited overview of the network. Only concepts can be browsed, not documents. This is also the case in the systems described by McMath et al. (1989) and Lucarella and Zanzi (1994).

For the browser, we envision a system that combines the visual browsing interface from hypertext systems with the domain representation of IR systems. Thoth-II (Collier, 1987) is such a system; it handles documents and concepts similarly. The system is similar to ours, but it has important shortcomings: the user easily becomes disoriented because the system lacks navigation aids, typing of nodes and links is limited, and because the system is specially designed for simple hypertext browsing, there is no provision for the inclusion of other kinds of documents. The drawing algorithm does not allow cycles; to avoid them, nodes are duplicated when necessary. Another system with a visual interface for browsing documents and domain representation is LyberWorld (Hemmje et al., 1994). It is more limited than ANS (which is described in the following sections) because only document-term links (and vice 
versa) are allowed. This forces the user to browse from document to key term to document to key term, and so forth. A 3D interface is used to visualize the network. Again, because the drawing algorithm does not allow cycles, nodes are duplicated to avoid cycles when necessary.

Access to a document involves retrieval and presentation. The information required to do this can be considered as the fourth kind of meta-information in ANs. In our approach we use available programs, while conforming to the addressing conventions of the Web. The Web offers a uniform addressing convention, whereby each document is assigned a unique address, called Uniform Resource Locator (URL). A URL consists of a transfer protocol, the Internet address of the host machine, and a path that uniquely determines a document on the host machine - usually the directory and the filename of the document (Berners-Lee et al., 1994; Fielding, 1995).

The browse and query mechanisms yield the URLs of the documents found. Once the URL of a document is known, the document is retrieved with an existing transfer program. Subsequently, an appropriate DAP is activated for presentation of the document.

\subsubsection{The Architecture of an ANS Implementation}

An operational implementation of ANS is considered to consist of two distinct parts: on the one side there is the network system proper, and on the other side there are the information sources containing the documents to be searched for and to be accessed. In Figure 3.1 these parts are labelled 'ANS' and 'external information sources' respectively; in the figure they are separated by a dashed line.

The ANS part is functionally divided into two subparts. The first subpart consists of a piece of software called the ANS-S, a collection of server data (ANS-SD) and a number of input and output facilities. This subpart, called the server for short, will be discussed extensively in Section 3.4. The second subsystem is a database system containing all relevant search data about the documents in the external information sources. It is called the Information Network for short. It has the usual functional components of a database system: the collection of individual facts or fact base (ANS$\mathrm{FB}$ ), the conceptual schema (ANS-CS), the metaschema (ANS-MS), and the software by means of which the contents of all three collections of data is managed, called the ANS manager (ANS-M). The database system is extensively discussed in Section 3.3.

The documents in the external information sources may be of any medium kind. This means that users can for instance view movies and images as easily as text documents. Next to the documents about which the ANS-FB contains information, the external information sources may also contain DAPs. Note that 'external' only means: being outside the ANS part. The actual physical location of a document may vary from residing on the computer system that hosts the ANS implementation itself to any place in the world. In the latter case a transmission link must of course be established. 


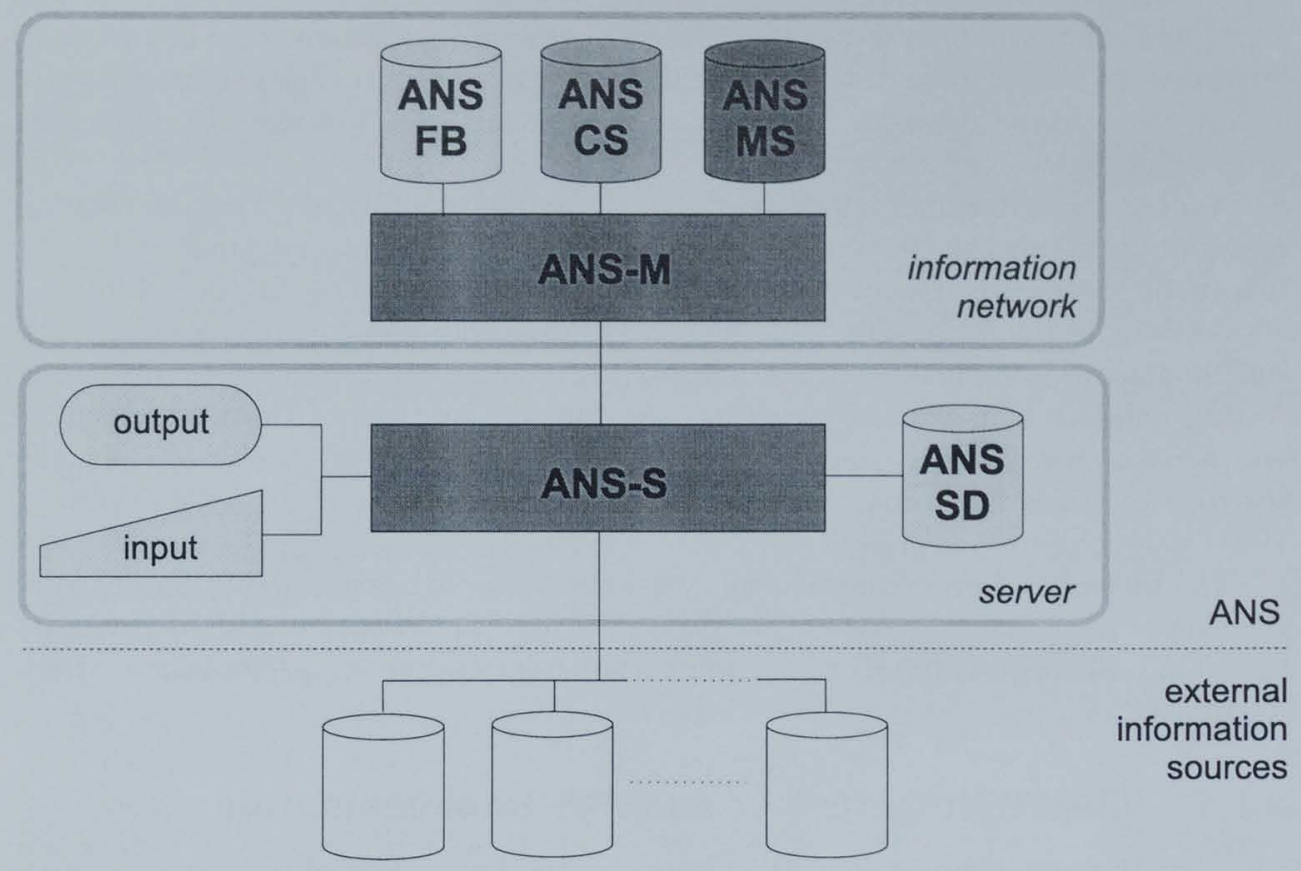

Figure 3.1: The ANS architecture.

An installation of the ANS software and the corresponding data files on a particular computer system, together with the communication links to external information sources, is called an ANS site. The persons that make use of the ANS search and access facilities are called the (site) users. The users get access to these facilities by means of the input and output devices, as available at the site. Next to the users there is a person (or a role of one of the users) called the (site) administrator. It is the responsibility of this administrator to provide and control the contents of the ANS-FB. More specific responsibilities are the provision and control of the conceptual schema (ANS-CS) and the server data (ANS-SD). In Figure 3.1, the symbols representing them are filled in light grey. To contrast them with the fixed components of ANS (thus the ANS-MS, ANS-M, and ANS-S), the latter are filled in dark grey. These fixed components are the same for all ANS sites, that is to say, in a functional sense. Regarding ANS-M and ANS-MS an appropriate standard RDBMS (Relational DataBase Management System) is envisaged.

An instance of using an ANS site by a user is called a session. During a session the user may perform one or more of the following tasks:

- Querying the ANS-FB so as to obtain answers to specific questions about the documents in the external information sources 
- Querying the ANS-CS so as to know what kind of data are contained in the ANS-FB, for instance, for the sake of adding new data to the ANS-FB

- Browsing through the contents of the ANS-FB, for instance, to search for documents contained in the external information sources

- Requesting access, through the service of ANS-S, to a particular document in the external information sources, and inspecting its contents by means of the appropriate output device(s)

The software system ANS-S may be considered the core of an ANS site because it is the component with which the users interact directly. Since it is a fixed component too, it has a similar interface on all implementations, and thus users can in principle easily switch between different ANS sites. Prevailing differences then will particularly stem from differences between the standard RDBMSs chosen for the actual realisation of the ANS-M and ANS-MS. These differences will however rarely raise serious problems because they are all based on the Relational Model.

It is important to note that two or more copies of the same document cannot be distinguished in the ANS-FB. However, it is necessary that they are distinguished by ANS-S. As a consequence, the user will be aware of the existence of one document only, whereas ANs-S 'knows' all copies and so will be able to present to the user the copy that is, for instance, cheapest or fastest accessible.

Concluding, we think that the ANS architecture as presented above offers a fair balance between user friendliness on the one hand and ease of implementation and administration on the other hand.

\subsection{The ANS Information Network}

As we mentioned already in the introduction of this chapter, the choice for the name 'Information Network' goes back to the start of the ARCHIMEDES project, when a semantic network was thought of as the means of structuring the factual knowledge to be used in assisting the ANS user in finding what he is looking for. Semantic networks however are not so rigorously defined that reliable management software can be easily built. Therefore we have chosen the well-known concept of the relational database (see e.g., Date, 1990) as the basic vehicle for managing the ANs Information Network. Any good RDBMs then will provide the necessary functionality regarding the storage and retrieval of the network elements. These elements are divided into three collections: the fact base (ANS-FB), the conceptual schema (ANS-CS) and the metaschema (ANS-MS). They will be described and discussed in the three subsections below. This will be done by using the fact-oriented modelling technique DEMO/FM which is extensively described by Dietz (1994). The application of DEMO/FM yields conceptual schemas that can straightforwardly be transformed to database schemas in the fifth normal form according to the Relational Model. 


\subsubsection{The ANS Factbase (ANS-FB)}

The ANS-FB contains all original data considered to be relevant (by the site administrator or by the user) for providing the search service of ANS. The core data in the ANS-FB are the existence facts of the documents in the external information sources. The existence fact of a document is the fact that the document is known to the ANS site considered. Such a fact can be expressed by a sentence like: "There exists an instance of the type document with document number ...". The document number is assigned by the site administrator and is therefore a local identifier. It is advisable however to assign identifiers that are common for the document type considered, for instance, to use ISBNs for books.

Next to these core data, ANS-FB may contain all sorts of data that are somehow related to the documents. Examples of such data regarding, for instance, an article, are the author(s), the journal in which it is published, the volume and number of the issue in which it appeared, and the date of appearance of that issue. Examples of such data regarding, for instance, an X-ray radiograph, are the name of the radiologist, the name of the patient, and the date and time of exposure. In addition, ANS-FB may contain thesaurus information. Thesaurus information consists of key terms and relations between these words. An example of two words and their relation is the following: Pharynx is synonymous to Throat.

Which data can be stored (and retrieved) and which cannot, is completely determined by the conceptual schema of the database (cf. Subsection 3.3.2).

The contents of ANS-FB can be inspected through the standard access means of the chosen RDBMS, for instance, by means of some SQL version. Next to that, it can be accessed by the ANS query mechanism and presented graphically on the screen of the user work station by means of a piece of software called the browse mechanism. The query mechanism and the browse mechanism are part of the ANS server, and will be described in Section 3.4 .

\subsubsection{The ANS Conceptual Schema (ANS-CS)}

As mentioned already in the previous subsection, the ANS-CS of a site determines what kinds of data can be stored in and retrieved from the site database. An ANS-CS, like any conceptual schema, therefore is first of all a specification of the fact types that are considered relevant. Next to these, it contains also the static constraints on the database states and the dynamic constraints on the database state transitions that hold.

The ANS-CS designed for a site has to be transformed to a relational database schema in accordance with the possibilities as offered by the RDBMS chosen. Constraints that cannot be expressed in the Data Definition Language of the RDBMS have to be dealt with in additional application software.

As an example of an ANS-CS, Figure 3.2 shows a portion of a conceptual schema that might have been designed for data concerning scientific articles, expressed in 


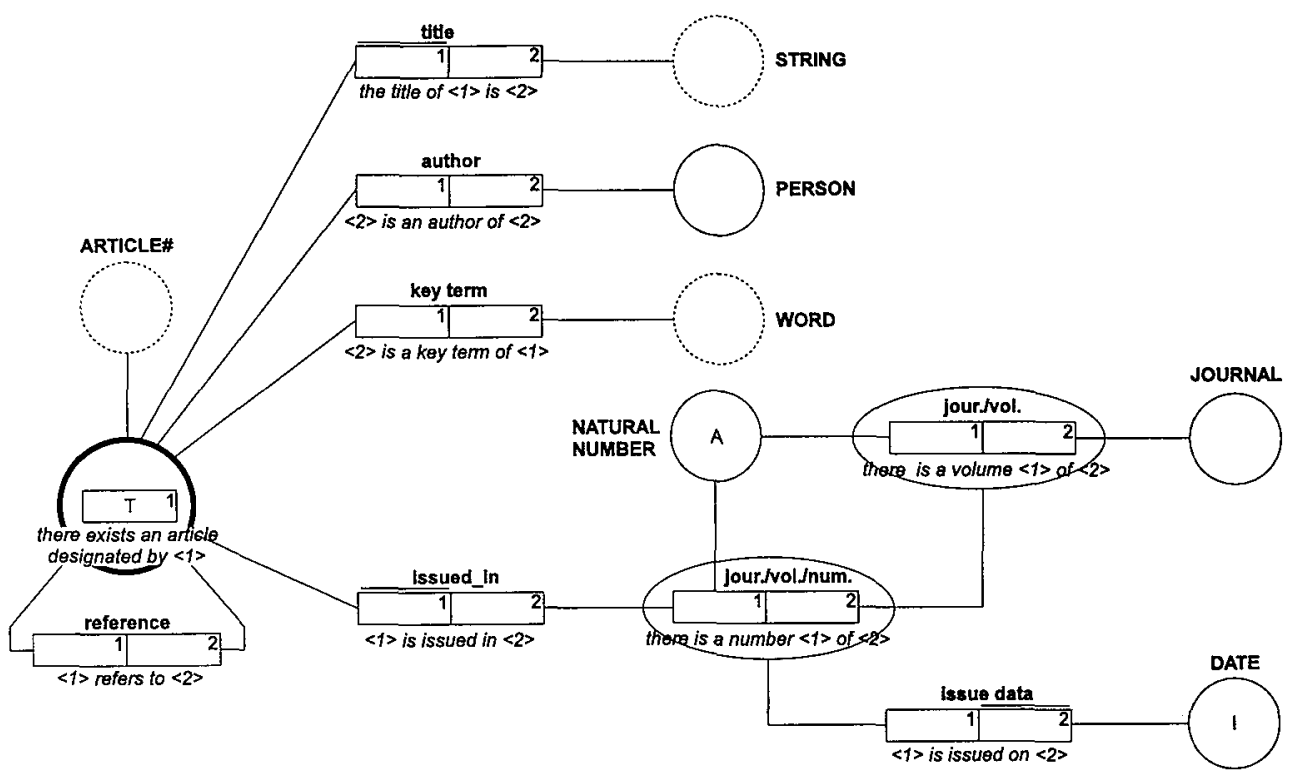

Figure 3.2: An example conceptual schema.

the DEMO/FM diagramming technique. Its meaning will be explained hereafter.

Fact types fact type are represented by (sequences of adjacent) rectangles: a unary fact type by one single rectangle, a binary fact type by two adjacent rectangles, a ternary fact type by three adjacent rectangles, and so on. The fact-type names are printed directly above the rectangles. Each rectangle represents the referents in a particular role in facts of the fact type considered. For instance, in facts of the type author the left rectangle represents the role of the article of which someone is author, and the right rectangle represents the role of the person that is an author of the article. To be independent of the drawing sequence of the rectangles, they are numbered from left to right. These numbers occur also between the bracket pairs ' $<$ ' and ' $>$ ' in the sentence beneath the sequence of adjacent rectangles. This sentence expresses in natural language the meaning of facts of the fact type specified. (Note: actually, the sentence is a sentence type because the nominal parts are variables.)

To exemplify the preceding, let us take the sentence "the person designated by the person name 'A. Turing' is an author of the article designated by the article\# ' 123456 " " as a possible instance of the sentence type " $<2>$ is an author of $<1>$ ". Likewise, the sentence "the title of the article designated by the article\# ' 123456 ' is 'Computing Machinery and Intelligence'" is a possible instance of the sentence type "the title of $\langle 1\rangle$ is $\langle 2\rangle$ " belonging to the fact type title. These two sentences together are a precise and unambiguous expression of what in common language would be expressed as "A. Turing is the author of 'Computing Machinery and Intelligence" . 
The allowable states of a FB are guarded by so-called static constraints. Probably the most basic kind of static constraints are the reference constraints. They are expressed by a line that connects every rectangle with a particular circle or oval. Circles or ovals represent extensions of fact types. So they are classes of objects (in case of unary fact types) or classes of object aggregates (in case of $n$-ary fact types). Collectively these classes are called object classes. A line from an object class to a rectangle expresses that the object class is the reference class of the referents in the particular role of the fact type that is represented by the rectangle.

If an object class is considered to be given, that is, to exist already or to be provided externally, it is represented by a straight circle and named by a noun in capital. Examples are PERSON and JOURNAL. Otherwise, that is, if its population is created by the user, it is represented by a circle or an oval that encloses a rectangle or a sequence of adjacent rectangles and its name is written in regular characters. Examples are article and jour./vol./num.

The fact type article is an existence fact type. The signs by which instances of the fact type article are designated, are taken from the sign set ARTICLE\#. ${ }^{3}$

A straight line above a rectangle expresses a uniqueness constraint. It means that in any extension of the fact type, referents in that role may only appear once. For instance, an article may only have one title, and an article may only be issued once. $^{4}$

\subsubsection{The ANS Metaschema (ANS-MS)}

A conceptual schema may itself be viewed as a database. This view is very fruitful since it gives rise to the existence of a 'higher' conceptual schema of which the previous one is an instance, in the same way as any database is an instance of its conceptual schema. The 'higher' conceptual schema is usually called the metaschema. An advantage of explicitly formulating the metaschema of a conceptual schema is that one has a thorough criterion by which to verify the syntactical correctness of the conceptual schema. Another advantage, more of a theoretical kind, is that one is able to recognize two or more conceptual schemas as being of the 'same kind', that is, obeying the same metaschema.

For the purpose of ANS a metaschema is designed that seems to fulfil all needs regarding the searching for documents and the accessing of documents. The main difference with current metaschemas is the existence of document types. An example of a document type is the type article in Figure 3.2. On the one hand, articles are things, like persons and journals, about which one can gather and communicate

\footnotetext{
${ }^{3}$ The character '\#' as the last character of a sign set name indicates that the sign set is the set of preferred names. Elements of other sign sets may be declared to be a synonym of a preferred name.

${ }^{4}$ One may of course choose to drop this constraint, so as to let an article be issued in more than one journal or in more than one issue of the same journal; it is just a matter of design of the conceptual schema.
} 


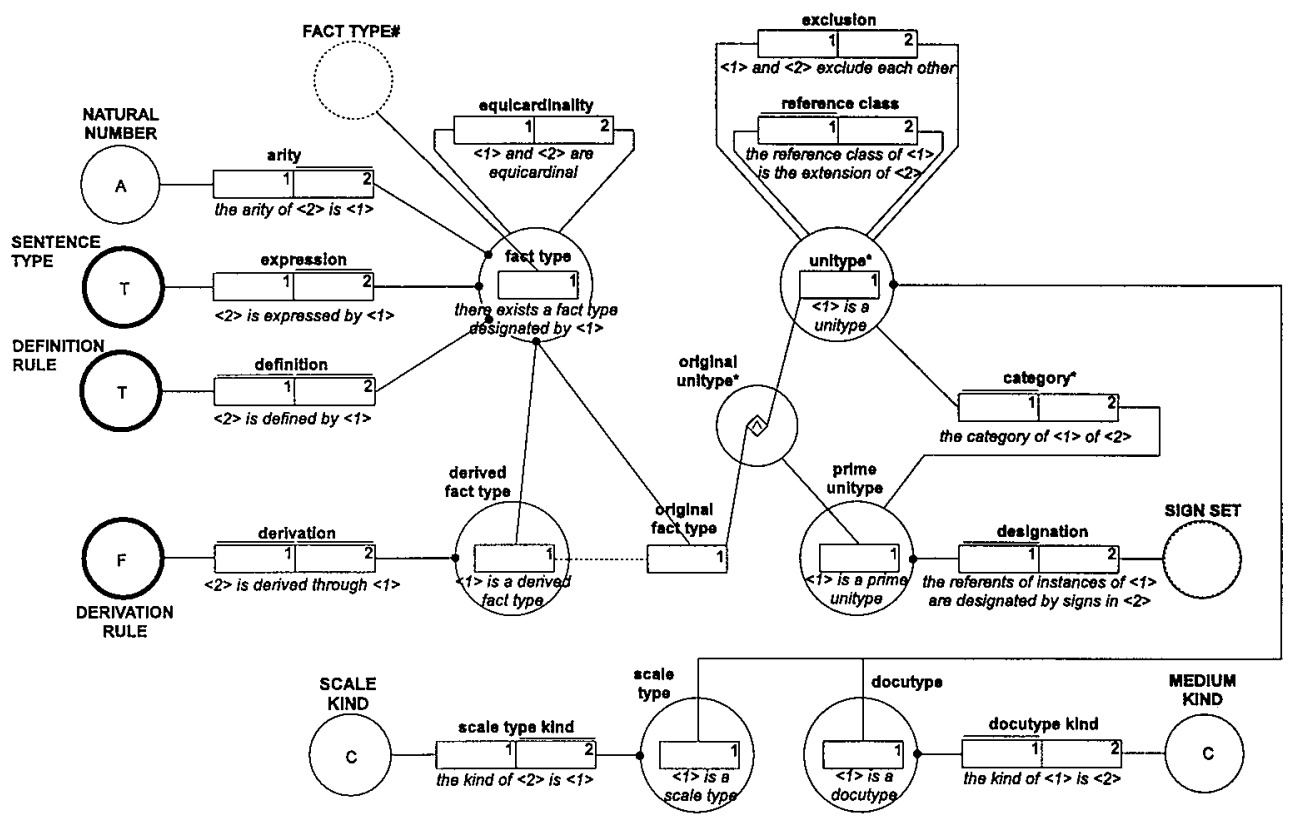

Figure 3.3: The ANS metaschema.

knowledge (facts). On the other hand, they are, or better: they contain, information themselves. To indicate this special type of things, the object class symbol (the circle or oval) is drawn bold.

The complete metaschema of ANS is shown in Figure 3.3. We will not expound the ANS-MS fully, but limit ourselves to elucidate some parts of it below.

The unary fact type (or unitype) designated by article (in Figure 3.2) is created firstly as an instance of the fact type called fact type. The name article is taken from the sign set called FACT TYPE\#. The arity of article is declared to be 1 , which makes article a unitype (the asterisk after the fact-type name unitype means that it is a derived fact type. The derivation is taken from facts of the type arity, as shown for the fact type article). In the conceptual schema of Figure 3.2 this is expressed by the single rectangle.

The fact type article is also declared to be an original fact type and subsequently to be a prime unitype. A unitype that is not a prime unitype is a specialisation of a (prime) unitype, and therefore inherits the (preferred) name from the prime unitype. A prime unitype is defined to be a unitype that is not a specialisation of another unitype. This is expressed in Figure 3.2 by the fact that it has no reference class, but instead a sign set (ARTICLE\#) linked to it from which signs are taken to designate articles. This link represents an instance of the fact type designation in the metaschema. Lastly, the fact type article is declared to be a docutype, indicated 


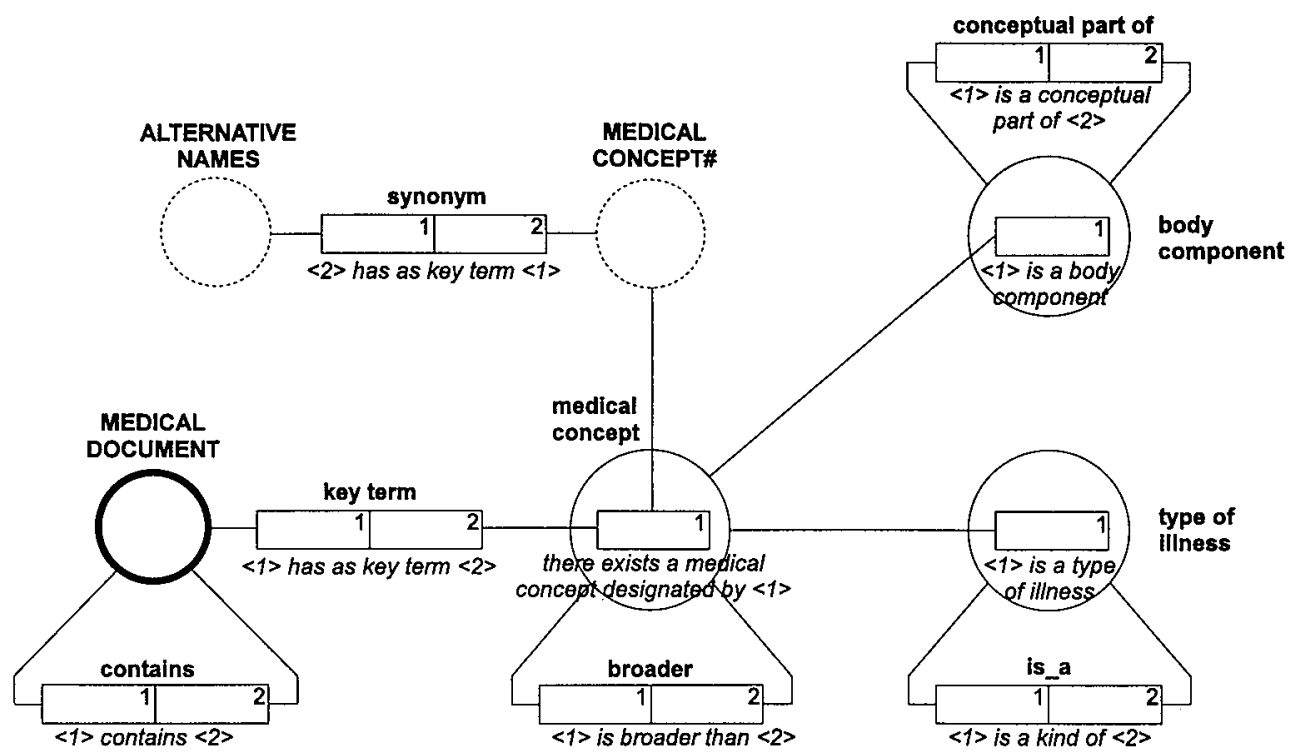

Figure 3.4: Conceptual schema of the medical example.

in Figure 3.2 by the bold circle. The document kind of article is declared to be text, indicated by the letter ' $\mathrm{T}$ ' in Figure 3.2.

The class MEDIUM KIND in Figure 3.3 is itself (the extension of) a scale type, namely of the kind categorical (indicated by the letter ' $\mathrm{C}$ '). A categorical scale is a scale without any relationship between the scale values, it is just an enumeration of possible values. Other examples of scale types are the class NATURAL NUMBER of the kind absolute (A), in both Figure 3.2 and 3.3, and the class DATE of the kind interval (I) in Figure 3.2.

\subsection{The ANS Server}

Having discussed the external information sources in Section 3.2 and the ANS Information Network in Section 3.3, we now shift our attention to the ANS server, which provides the user with an interface to the information contained in the ANS-FB and in the external information sources. The core of the ANS server is the software called ANS-S. The user interacts with ANS-S using for instance a keyboard and a pointing device as input devices, and a video monitor (screen display) and audio monitor (speakers) as output devices.

Below we show an example drawn from the medical domain to illustrate the function of the server. The conceptual schema pertaining to this example is shown in Figure 3.4. The majority of the medical concepts and alternative names are 
taken from uMLs. The documents originate from the Virtual Hospital ${ }^{5}$, a collection of medical multimedia textbooks. It is available on the Web.

The user is able to inspect the contents of ANS-FB with two tools contained in ANS-S: the query mechanism and the browse mechanism. The query mechanism allows the user to query the ANS-FB. Typically the user will choose the query mechanism when he has a fair idea of what information he needs. For instance, which documents are about croup and influenza? However, in Section 1.3 we concluded that the browse mechanism can also be used in these cases. Queries are expressed in a formal language, the query language. We currently use a query language that allows combinations of key terms with the Boolean operators. For more complex queries, concerning fact types, SQL can be used as offered by ANS-M.

The results of a query are shown to the user in the form of a list of facts. Any of the referents of these facts can act as a starting point for browsing.

The browse mechanism or browser lets the user browse through a graphical representation of the facts in ANS-FB. Typically, the user will choose the browser when he has only a vague idea of the information he is looking for, or when he does not know the exact key terms. An example of the browser output is shown in Figure 3.5.

A fact is depicted in this figure by two nodes representing the referents and a line representing the relation established by the fact type. Along the connecting line the fact-type name is shown. Following the line in the reading direction, the first node corresponds with the left referent and the second node with the right referent. 'Left' and 'right' correspond with the places in the expression sentences as printed beneath the fact types in Figure 3.4. The Information Network part shown in Figure 3.5 consists of a text document (Croup Syndromes, dark box), an image document (Diphteria \& Pseudomembrane, grey box) and key terms (white boxes). Note that a key term here is either a medical concept or an alternative name (cf. Figure 3.4).

Browsing is done as follows. The user starts with one node (representing a document or a key term) and has the related nodes shown by a single command. Selection of a node and the subsequent presentation of related nodes can be repeated, thereby expanding the visible part of the Information Network. However, other nodes may be removed by the system, because we experienced that more than 15 nodes may easily clutter the display and disorient the user. We therefore present only a limited number of nodes at a time. The exact number depends of course on the particular $\mathrm{FB}$ in use.

The positions of the nodes on the screen are determined by the spring embedder algorithm (Eades, 1984) but it can also be viewed as a variation on the technique of multidimensional scaling (Schiffman et al., 1981). This technique translates information about 'distances' between pairs of two nodes (in our case the number of

\footnotetext{
${ }^{5}$ The Virtual Hospital is an initiative of the Electric Differential Multimedia Laboratory, Department of Radiology, University of Iowa College of Medicine. It can be found on the Web with URL: http://vh.radiology.uiowa.edu/
} 


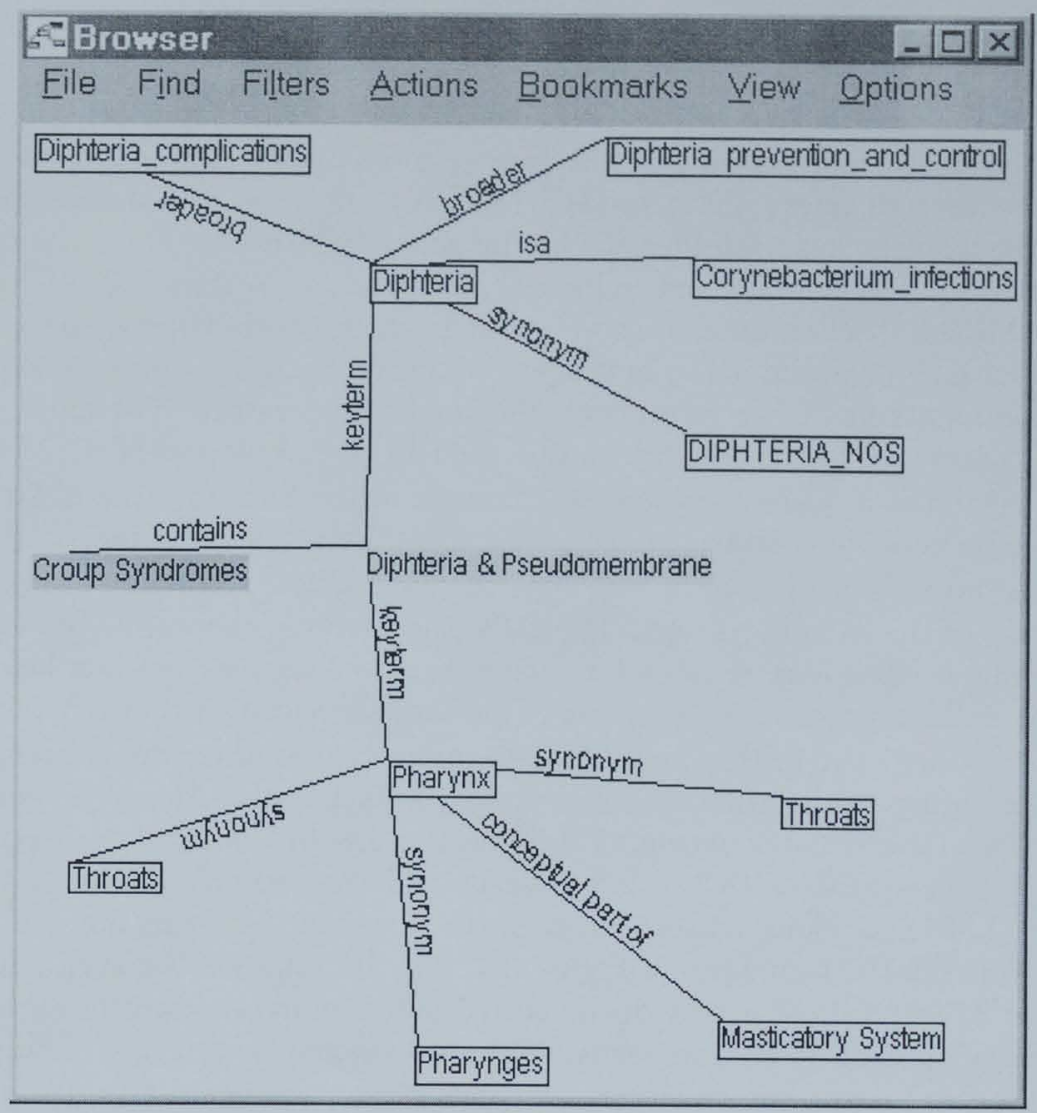

Figure 3.5: Browser view of the ANS-FB part of the medical example.

nodes visited when traversing the Information Network from the first node to the second one) to locations on the screen.

The user may request the content of nodes:

- For document nodes ANS-S then retrieves the document and starts the appropriate DAP for presentation.

- For key term nodes the content consists of a definition or description of the key term. On requesting the content of a key-term node, the definition is shown to the user.

An important instrument to prevent cluttering is the filter mechanism. It enables the user to exclude particular fact types from displaying. The link-type filter provides the user with a list of all available relations (link types) in the FB. The user can 
Table 3.1: Table view of the contents of the browse window in Figure 3.5.

\begin{tabular}{lrr}
\hline Medical concept & Synonym & Alternative names \\
\hline Pharynx & & Throat \\
Pharynx & & Throats \\
Pharynx & & Pharynges \\
Diphteria & & DiPHTERIA_NoS \\
& & \\
\hline Medical document & Key term & Medical concept \\
\hline Diphteria \& Pseudomembrane & & Pharynx \\
Diphteria \& Pseudomembrane & & Diphteria
\end{tabular}

\begin{tabular}{lll}
\hline Medical document & Broader & Medical document
\end{tabular}

Diphteria

Diphteria

Diphteria_prevention_and_control

Diphteria_complications

\begin{tabular}{lll}
\hline Type of illness & Is $\mathbf{A}$ & Type of illness
\end{tabular}

Diphteria

Corynebacterium_infections

Medical document

Contains

Medical document

Croup Syndromes

Diphteria \& Pseudomembrane

\begin{tabular}{lrr}
\hline Body component & Conceptual Part Of & Body component \\
\hline Pharynx & & Masticatory_System
\end{tabular}

select which relations should be ignored by the system. Filtering out relations in this way reduces the number of displayed links and nodes. For instance, the user may filter out the isa and part of link types. The node-type filter works in a similar way. The user can select which node types should be ignored, for instance the book and keyterm node types.

Figure 3.5 and Table 3.1 illustrate the advantage of depicting facts from ANS-FB in the browse mechanism over the traditional database table view; especially when many fact types with relatively few instances are concerned, the browser gives an easier and quicker insight into the information presented.

Using only isa links McMath et al. (1989) provides the user with a graphical 
representation of the domain knowledge, disregarding facts concerning documents. The graphical browser of Rožić and Dimec (1994) is used to find key terms for composing a query. However, it only shows the direct neighbours of a node.

The structure of a hyperdocument is merely shown partially by the ANs browse mechanism because the browse mechanism takes documents that consist of one atom (see Subsection 3.1.1) as a whole. Therefore, only inter-document links can be shown (and of course only those that were introduced to the ANS-FB). However, intradocument links can be shown if they are turned into inter-document links, that is, by promoting the document parts into documents.

A DAP runs independently of ANS-S and is not part of ANS; it is a stand-alone program that can be called from another program. In that way we ensure that ANS will not be outdated when in the future new document formats are introduced; all we need to do is to add the information about the corresponding new DAP to ANS. Incorporation of Java content handlers (Gosling and McGilton, 1995) will allow us to retrieve a DAP that is not present on the local host from a remote location. In that case the DAP is retrieved as if it were an ordinary document, given its address and a transfer protocol. Note that since we consider databases to be documents, one may access an ANS-FB contained in an external information source; thus an ANS-FB can contain pointers to other ANS-FBs in related domains.

Users of other programs may exploit ANS for supplying background information. For instance, a user of an expert system may consult ANS to find the answer to a question asked by the expert system. If ANs is started by a program instead of a user, the application can specify whether the query mechanism or the browse mechanism must be started initially, and with which key terms or documents.

For performing its tasks ANS-S needs the following supplementary data, stored in the system data (ANS-SD):

- For each document the document kind (audio, video, etc.) and the available formats are required - because a document may be available in multiple formats (e.g., ASCII, WP 5.1, $\mathrm{LAT}_{\mathrm{E}} \mathrm{X}$ ).

- For each copy of each document the location must be known. If the document is not on the site, it is retrieved using its URL.

- Information on how specific fact types should be depicted in the browser (the shape, the colour, the lay-out) is required. For instance, to prevent disorientation of the user during browsing, facts concerning documents are presented visually differently from facts concerning key terms.

- For each DAP its location and calling convention must be known; the ANS-S must know where to find each DAP and how to call it.

- For each format the name of the DAP preferred by the user for that format must be known. For instance, one user may prefer to view documents in WP 5.1 
format with the Word Perfect 6.0 program, another user may prefer to view these documents with MS Word 6.0.

\subsection{An Informal Evaluation}

To examine the effectiveness of ANS, a group of four health sciences students was asked to search for information about the Ebola virus. The test was part of a class in which information about the Ebola virus had to be collected from various sources. The students were asked to find general information about Ebola and information about its epidemiology. The Information Network used consisted of 100,000 key terms from the 1992 version of UMLS and 300 Medline records about the Ebola virus.

It turned out that the students became disoriented when they tried to go back to a previous situation and found that the graph layout had completely changed. They used the history list to go back, and expected to see previous situations, but the history list only reproduces previously clicked nodes. Another problem was the usage of the filter mechanism. The users were reluctant to use the mechanism, because they found it too confusing. This resulted in even more disorientation, because often too many link types were visible. Nevertheless, the students found working with ANS a positive experience.

\subsection{Chapter Conclusions}

In this chapter we introduced the ARCHIMEDES Network System. It aims to alleviate two problems that arise when large amounts of information become available: the search problem and the access problem. To resolve these problems we assumed the information to be contained in units called documents. Consequently the notion of document was defined and elaborated on (in Section 3.1).

To address the search and access problems separately we conceived of two functional parts of ANS: the network and the server. To address the search problem the network part contains information about the documents as well as a thesaurus. The server lets the user search for documents in the network by means of a query mechanism and a browse mechanism. The server also provides access to documents by retrieving them and presenting these contents using an appropriate DAP.

A prototype installation of ANS was built. As far as the server is concerned the implemented parts are:

- ANS-S; however, retrieval of remote documents is limited to documents on the Web and a document can be stored at one location only

- The browser mechanism 
- An elementary query tool that deals with Boolean queries

As far as the network is concerned, the implemented parts are:

- ANS-FB: It is not realised with an RDBMS but is implemented in our own data structures, as an inheritance from preliminary research; ANS-CS and ANS-MS are not explicitly represented.

- Several ANS-FBS: The largest one contains information about the medical domain. The terms were taken from the Unified Medical Language System and the documents from the Medline medical abstracts.

A future improvement that is not further discussed in this thesis concerns the query mechanism. A device is being implemented by Van der Pol (1996) for the composition of queries, serving as a search intermediary for the query mechanism. The device consists of (1) a language and procedure allowing users to specify information needs, (2) a procedure for finding the concepts that meet a given specification, and (3) a procedure that automatically formulates a query from the concepts found. In the procedures the domain representation is used.

An implementation of ANS was informally evaluated on a network with 100,000 nodes. As a result, several limitations were identified that will be addressed in the next chapter. 


\title{
Chapter 4
}

\section{Implementation of the Archimedes Network System}

\begin{abstract}
This chapter is an updated and abridged version of: Wiesman, F. and Hasman, A. (1997). Graphical information retrieval by browsing meta-information. Computer Methods and Programs in Biomedicine, Vol. 53, No. 3, pp. 135-152.
\end{abstract}

In the previous chapter the design of ANS was discussed and a first prototype was presented. An informal evaluation (Section 3.5) revealed several shortcomings of the prototype. These shortcomings are addressed in this chapter in Section 4.1, leading to our final implementation. Moreover, a more restricted Information Network is defined. Several implemented Information Networks are presented in Section 4.2. The communication between ANS and the DAPS is discussed in Section 4.3. A detailed description of the ANS browser is given in Section 4.4 and the last section contains the chapter conclusions.

\subsection{The Archimedes Information Network}

The Information Network contains domain knowledge and document knowledge. The domain knowledge is represented by a thesaurus. In IR systems this is the traditional term for a domain representation. A typical thesaurus contains only a few relations, such as is a and synonym. For a thesaurus containing more than a few hierarchies the term 'semantic network' might be more suitable, although it suggests the use of artificial-intelligence search-and-inference techniques; the latter techniques are not a part of our research. For thesaurus standards we refer to ISO (1986) and Jansen (1993), and for an account on semantic networks to Maida and Shapiro (1982).

The concepts of a thesaurus are represented as concept nodes (or key terms) in the Information Network. Relations between concepts are expressed as links between 
concept nodes. Documents are represented by document nodes. They contain information about the location of the document contents. Key terms describing the content of a document are represented in the Information Network by links to the appropriate concept nodes. Specializations of the concept node type and document node type may be defined so the user can distinguish subtypes of the concept and document types (e.g., book is a subtype of the document node type).

The software prototype requires the author of an Information Network to specify all nodes and their relations in an ASCII file which is later compiled to a custom-made database by a special network compiler. Although this database offers fast access to the data, which is important for our system, the drawback is that the compilation process is time consuming. On our Pentium 90 machine, a network of 100,000 nodes takes a day to compile. However, the compilation has to take place only once.

The ASCII file of the Information Network consists of five elements with the following syntax (meta-characters are ' $<$ ' and ' $>$ ', denoting a placeholder, and '[' and 'l', denoting an option; as separator ',' is used).

1. A document:

$\mathbf{d}(<$ document name $>,<$ file $>,[<$ tag $>])[(<$ node type name $>)]$

where document name is the name of the document to be used on the screen, file is the file where the document is stored. The file name extension determines which DAP is used to present the content of the document. A special case is the extension .url: it denotes a Web document id (URL) (Berners-Lee et al., 1994; Fielding, 1995). With the node type name a specialization of the node type document can be assigned. If it is omitted, the node type is by default document. The optional tag is used to specify a position in a file. File names, tags and DAPs are discussed further in Section 4.3.

2. A concept:

c $(<$ concept name $>)[(<$ node type name $>)]$

where concept name is the name of the concept to be used on the screen and node type name its node type. With the node type name a specialization of the node type concept can be assigned. If it is omitted, the node type is by default concept.

3. A link:

$1(<$ source $>,[<$ link type $>],<$ destination $>)[(<$ node type name $>)]$ where source is the name of the source node, link type is the type of the relation between the two nodes, and destination the name of the destination node. Document and concept nodes may be both source and destination. If the link type is omitted, the link is of the anonymous link type (which means no link type name will be shown during browsing). If specified, the node type name is assigned to the destination node. In practice this is used when links are made from one source to multiple destinations. The source node type needs to be defined only once. When (some of the) destination nodes were not yet 
defined they are now by specifying their node type names. If the node type of the destination is not defined, it is by default the type concept.

4. A node type appearance:

$\mathbf{n}$ (< node type name $>,<\mathrm{R}>,<\mathrm{G}>,<\mathrm{B}>$ )

where node type name is the name of the node type and $R, G$ and $B$ determine the RGB (red, green, blue) colour value of the nodes of that type in the browser. If a node type is used for which no appearance is defined, a default colour is used. For the node types document and concept also default colours are defined, that can be changed.

5. The item separator:

a $(<$ separator $>)$

where separator is the item separator to be used in the remainder of the file.

In the previous definitions the default value ',' was used.

If a document definition is preceded by an empty line, all concepts in concept definitions below the document definition - until the next empty line - are linked to the document with the link type keyterm. As an example, a few lines of an Information Network are listed below:

1. $a(\mid)$

2 .

3. d(Wiesman $97 \mid \mathrm{cmpb} \cdot$ tex $\mid$ ) (article)

4. c(information retrieval)

5. c(browsing)

6. c(ANS)

7.

8. $\mathrm{n}(\operatorname{article}|255| 0 \mid 128)$

9.

10. I(Wiesman97/has author|Wiesman, F.) (author)

11. I(ANS|instance|IR system)

12. I (information retrieval/synonym|IR)

13. I (methods | programs) 
Line 1 defines the I symbol as separator. Line 3 defines a document with the name 'Wiesman97'. It is of subtype article and its content is stored in the file cmpb.tex. The three following lines define its key terms (which are all of node type concept). Line 8 defines the appearance of the node type article. By default, the appearance of the node types concept and document is defined. The last four lines are links that express the facts: "Wiesman97 has as author Wiesman, F.", "ANs is an instance of an IR system", "information retrieval is a synonym for IR" and "methods is related to programs". The last link is of the anonymous link type. The constituents of the facts are by default of node type concept, except for 'Wiesman, F.', which is of type author (a subtype of concept).

The database which results from compilation of the Ascir file consists of a data file and an index file. For each node a record of variable length is created in the data file from the ASCII file with the following information: node id, node type, number of incoming links, number of outgoing links, type and destination of each incoming link, and type and destination of each outgoing link. The index file contains the node names in alphabetical order. Given a node name, the system can locate the corresponding record with binary search using the index file. This is necessary when the user enters a node name for displaying in the browser. The destination (or source) of each link is the record address of the destination (or source) node; thus links are stored as record addresses and therefore directly retrievable.

Explanations of concepts are stored in a separate AScrI file. When during browsing the user double clicks on a concept node, its explanation, if available, is shown. Information on DAPs (Section 4.3) and filters (Subsection 4.4.3) are stored in a MS windows .ini file.

From the definitions above it follows that there are two predefined node types: concept and document, and one predefined link type: keyterm. The author of an Information Network can define more types. Common node types derived from document are chapter, book, and movie. Common node types derived from concept are author and title. Common link types are has author, kind of, causes. Node types and link types are important for the browser, because they influence the way a node or a link is displayed. Knowing the type of a node or a link can be helpful for the user while choosing a browsing path. More important is the possibility to hide specific node types and link types from the browser. This will be further discussed in Subsection 4.4.3.

\subsection{Implemented Information Networks}

The Information Network merely requires a domain representation of the topics discussed in the documents in the Information Network. Thus, a domain representation of the entire world is not necessary; different document collections require different domain representations. Any thesaurus or semantic network about any domain (e.g., law or economics) can be used. Because the Information Network is specified as an 
ASCII file, it is elementary to convert an existing thesaurus or semantic network to an Information Network. We built Information Networks of various sizes, containing information about various domains. Four of them are discussed in the following sections. The first aims at plain IR, the second one at browsing through hypertext, the third one at classification, and the fourth one at the integration of expert systems and IR systems.

\subsubsection{The Medline Information Network}

The largest Information Network we built contains information from the medical domain. It was constructed to investigate the suitability of ANs for large Information Networks and for the medical domain.

For the domain knowledge the 1995 version of the Unified Medical Language System (UMLS) (Lindberg et al., 1993a) was used. UMLS is an initiative of the National Library of Medicine (U.S.). It contains a large thesaurus comprising several existing thesauri used in the medical domain. We selected some 300,000 key terms and made several modifications to make the thesaurus more understandable for the user.

- Only English key terms were used; the French, Portuguese, and Spanish translations were discarded.

- Names of some link types were changed where they would not make sense to the users.

- Redundant links were discarded (e.g., caused by links are redundant because of the causes links).

- The user starts off browsing by supplying a key term to begin with. He must have the freedom to enter a key term as he likes, after which ANS substitutes the preferred form for the key term. Therefore, lexical variants of key terms were retained.

- The organization of synonymous key terms was changed. A key term in UMLS is represented by a set of strings, which are lexical variants of each other. One of the strings is marked as the preferred string. A concept is represented by a set of synonymous terms. One of the terms is marked as the preferred term. This is depicted in Figure 4.1. Since there is no need for the distinction between terms and strings in ANs, we represent a concept by the preferred string of the preferred term; the other strings are linked to the concept (see Figure 4.2).

The ASCI file for the UMLS part of the Medline Information Network was generated directly from the UMLS CD-ROM by a Perl (Wall and Schwartz, 1991) program. 


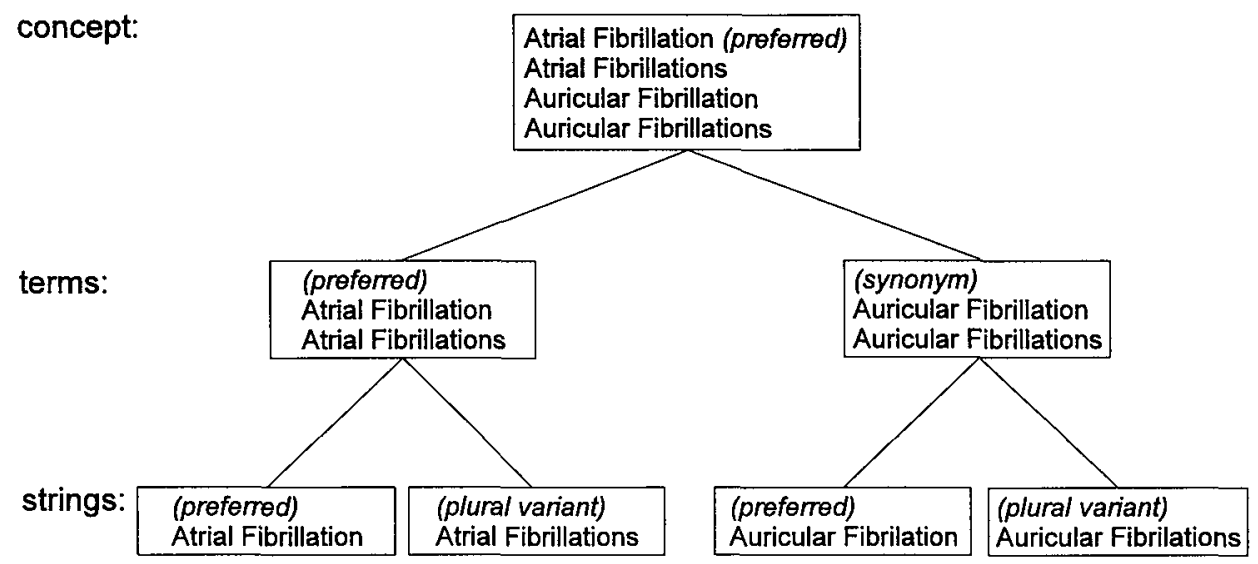

Figure 4.1: Organization of synonyms in UMLS (drawn after NLM, 1995).

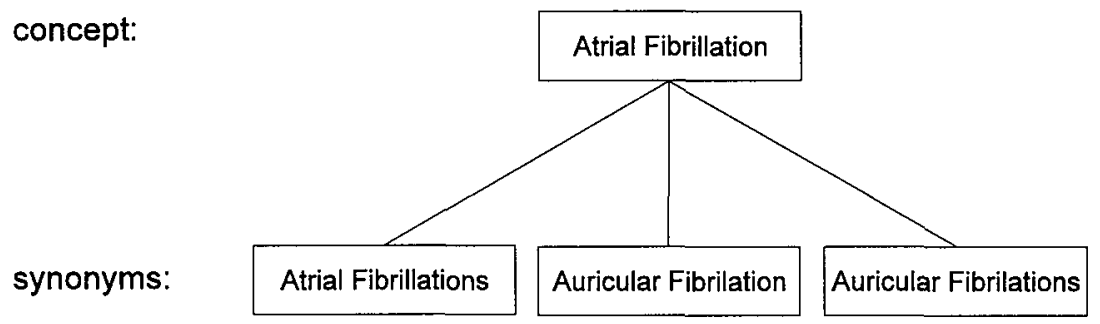

Figure 4.2: Organization of synonyms in ANS.

As for the documents, we downloaded the records of the biomedical literature database Medline that were added to Medline in January-March 1995 (approximately 36,000 records). The records were taken from a Silverplatter CD-ROM. Because they were stored in a proprietary format, they were converted to a single ASCII file using the Silverplatter IR system WinSpirs. Each time the user requests to see a document's content, a DAP extracts the record from the file, converts it to HTML and starts a HTML viewer to present the result.

Writing a small $\mathrm{C}++$ program that links the documents to the appropriate key terms (the indexing process) was trivial because the Medline records already contained a list of key terms from the MesH thesaurus, which is part of UMLS. The human Medline indexers indicate which of the key terms are the most important. In the Information Network this is reflected by the link type used to link the key term (a concept) to the document node; it is either the keyterm link type or the sec 
keyterm (abbreviation of 'secondary keyterm') link type. Key terms can be made up of two parts: a heading and a subheading. The subheading describes the topic more specifically. An example of a heading is: Epilepsy, an example of a subheading: drug therapy. In general, members of specific groups of headings have typical subheadings. For instance, drug therapy and etiology are typical subheadings for diseases, whereas adverse effects and therapeutic use are typical ones for drugs. In UMLS, subheadings are not linked to headings. The Medline records are however indexed with heading-subheading combinations. Since ANS does not provide ternary links, a new concept node is required for every heading-subheading combination. Where at least one document used a particular heading-subheading combination, a new concept node of subtype mainsub ${ }^{1}$ was created and linked to the constituting concept nodes. In the example above this resulted in the new node Epilepsy drug therapy with links to Epilepsy and drug therapy, and also to several document nodes.

Why would we use UMLS as domain knowledge when UMLS is a superset of the indexing thesaurus, MesH? Why not simply use MesH as domain knowledge? MesH consists of several hierarchies. UMLS adds extra links, resulting in a tangled hierarchy. Also more concepts are present. We expected that the extra links and concepts would make it easier for users to get to the concepts they are looking for. We also experimented with the UMLS semantic network, which is a semantic network separate from the UMLS thesaurus. It connects the top levels of the thesaurus hierarchies using rather abstract concepts (e.g., phenomenon or process and physical object). The UMLS semantic network proved to be too abstract for the Medline Information Network. Users would have problems understanding the concepts. Furthermore, they would rather choose less-abstract concepts as starting points for browsing. Therefore, the UMLS semantic network was not included in the final version of the Medline Information Network.

\subsubsection{The Virtual Hospital Information Network}

Another example of an Information Network that we created also uses UMLS as domain knowledge, but contains as documents a part of the Virtual Hospital, which is a multimedia book on the Web for medical education. The documents contain text, pictures, sounds and movies. In this case, indexing was done manually. The goal of this Information Network was to investigate the suitability of ANS for hypertext navigation and to demonstrate its ability to handle multimedia documents. Therefore, the emphasis in this network is on links between documents. In the Information Network, each Web page is regarded as a document. Several Web pages together also may constitute a document. A distinction is made between the part of link type (e.g., used between a chapter and its sections, or a section and its sound fragments) and the refers to link type (e.g., used between two documents of which one has a bibliographic reference to the other). This distinction entails extra information for

${ }^{1}$ This is shorthand for 'main heading - subheading'. 
the navigating user. However, in some occasions it is not straightforward to make the distinction between the two link types. A Web page may also contain a link to a position in the same Web page. ${ }^{2}$ These links cannot be represented in ANS. We elaborate on the hypertext issue in Section 4.4.1.

\subsubsection{The Paleopathology Information Network}

In paleopathology - the study of disease in ancient times as revealed in bones and mummies - the description of bones found during archaeological excavations is essential. In collaboration with the Department of Radiology of the University Hospital Maastricht, an Information Network was constructed that supports the paleopathologist in classifying fractures of long bones (Wiesman and Panhuysen, 1995).

The system supports the user in three ways:

- The classification features (e.g., side) and the feature values (e.g., left) are represented as concepts in the Information Network. The user can browse through this classification tree and mark appropriate feature values.

- Some features are illustrated with diagrams, because opinions or perceptions of users (and paleopathologic experts) on the concepts used may differ. For instance, the shape/direction of a fracture may be spiral, transversal, oblique, comminuted, or avulsion. To each of the shape/direction values a diagram (a document node) is linked that depicts the particular fracture shape/direction. To the concept shape/direction a diagram is linked that is an overview of the five shape/direction value diagrams.

- Pictures of example cases can help the user in deciding whether a particular feature value applies to the case under examination. Cases are present in the form of photographs and X-ray films that are linked together. Similar cases are also linked to each other. Pictures can be compared easily, because the DAP always shows a juxtaposition of the three pictures that were selected last.

A limitation of ANS for classification is the impossibility of specifying that particular feature values exclude each other. Consequently, it is possible for the user to end up with a inconsistent classification if he makes the wrong choices.

Summarizing, we can say that this network primarily aims at indexing rather than retrieval: the user has a fractured bone (a 'document') and wishes to find the proper classification (the 'key terms'). Of course, the application can be used for retrieval as well; pictures of classified bones can be added to the Information Network and retrieved on demand.

\footnotetext{
${ }^{2}$ This is accomplished by extending the URL with a fragment identifier (Berners-Lee and Connolly, 1995).
} 


\subsubsection{The Transnational Income Tax Information Network}

We examined the extension of rule-based expert systems with ANs. There are two applications: Firstly, the user of the expert system sometimes needs background information to answer a question of the expert system. ANS can help the user to find the information, especially if the Information Network contains links from the rule base to the background information. Secondly, if the rules are represented in the Information Network, the user will understand better why the expert system asks a particular question, because ANS visualizes the structure of the rules and the relations between the rules. Thus, it is an addition to the explanation facilities of expert systems (see, e.g., Lucas and Van der Gaag, 1991, Chapter 6).

An existing rule base was taken: it decides in which country someone who lives in the Netherlands but works in another country has to pay his income tax. The rule base was written in PLt, the language of the LITES shell (Span, 1994), and contains 72 rules. The shell was extended with a user command to start ANS with the current rule as starting point. A tool was written to generate from a PL+ rule base a corresponding Information Network. As we saw in Subsection 3.1.2, a knowledge base or rule base can be considered as a document of medium kind formula. In this Information Network every rule of the rule base is a formula document. The content of such a document is the text of the rule in the rule base. There are links from the rule to the condition and the conclusion part. Each of these parts has links to the identifiers of the variables used. This implies that for each rule the user can see with which rules it is related, on which variables it depends and which variable it influences. For each variable the user can see in which rules it occurs. Rules are linked to related case law, texts of related laws, and help texts from the original rule base. The links to the texts were added to the Information Network manually.

After informal experimentation we concluded that the visualization of rules may be important in educational contexts, where users of an expert system must gain insight into the rule base. Although ANS was useful for locating relevant information, the possibility of communication between ANS and the expert system shell did not seem to be of extra value.

\subsection{Document Access Programs}

To show the content of a document programs different from ANS are used, so called DAPs. They were introduced in Subsection 3.1.1. In this section we elaborate on the communication with DAPS. DAPs must be executable from the same host on which ANS is running. Which DAP is used to present the content of a particular document depends on the extension of the document's file name. The ANS initialization file specifies for each file extension the associated DAP. We recall from Section 4.1 that a document node has an optional tag. Depending on the tag, three different types of DAP or different ways of calling (tag commands) may be defined for a specific 
extension. A tag command is a command line option for a DAP, for instance, -1 for jumping to the line specified by the tag. The three tag forms that are distinguished are:

No tag: The DAP is started with the document file name as argument.

Numerical tag: The DAP is started with the numerical tag command, the tag and the document file name. This can be used, for instance, to show a particular chapter of a document by supplying the line number where the chapter starts as tag. The tag command would then be the command line switch for the DAP to go to the specified line.

Non-numerical tag: The DAP is started with the non-numerical tag command, the tag, and the document file name. This can be used, for instance, to show a particular section in a document that may be changed; jumping to a particular line may not work when lines are added or deleted, but doing a search to a specific text string is likely to succeed. The tag command would then be the command line switch for the DAP to search for the specified text string.

In the examples above, the files may be of the same type, but because one tag is numerical and the other is not, different actions can be taken. The extension url is treated in a special way: before starting the DAP the URL extension is removed from the file name.

Most Web browsers handle the mapping of a file name on a DAP in two steps: first the file extension is mapped on a MIME type (Borenstein, 1992) (e.g., .htm and .html are mapped to text/html). Second, the MIME type is mapped to a DAP. The two-step process is necessary in a client/server architecture where the client does not know which file extensions the server uses. This is not the case for ANs. Web browsers have no provision to pass tags on the command line.

Because ANS relies on programs not part of ANS to present documents, no problems arise when documents are added with a new document format; only a suitable DAP is required. Incorporation of Java content handlers (Gosling and McGilton, 1995) will allow us to retrieve a DAP from a remote location that is not present on the local host. In that case the DAP is retrieved as if it were an ordinary document, given its address.

A remarkable example of a DAP for ANS is ANS itself: an Information Network can be considered as a document in another Information Network. We constructed a small Information Network with as domain: 'Information Networks'. Its documents are various versions of the Information Networks constructed in the course of the ARCHIMEDES project. An Information Network can be inspected as any document by double clicking on its node in the browser. Its DAP, a new instance of ANS, is then used to present it. 


\subsection{The ANS Browser}

The most important part of ANS is the browser tool. It visualizes the Information Network and allows the user to discover information by browsing through the network of concepts and documents. ANs was implemented in $\mathrm{C}++$ and runs on MS windows 3.11 (and consequently on MS windows 95). As hardware a Pentium 90 machine with $32 \mathrm{Mb}$ RAM is used, although a $486 \mathrm{Dx} 66$ with $16 \mathrm{Mb}$ RAM has proven to be sufficient.

Subsection 4.4.1 shows a sample session with the browser and Subsection 4.4.2 discusses how the Information Network is visualized. Subsection 4.4.3 describes the various navigation aids that prevent the user of becoming disoriented during browsing. An extension to the browser that allows combination of key terms is discussed in Subsection 4.4.4.

\subsubsection{Browsing through the Information Network}

In this section we will look at an example of finding documents by browsing in the Information Network. The example is depicted in Figure 4.3. We describe a situation that is common in educational environments.

Assume we are looking for documents about a part of the ear. We do not know its name, but we do know it is related to a nerve. Also the name of this nerve is unknown to us. We start off with a list of all available key terms, ordered alphabetically. After we have typed 'ear', we can select the key term ear internal from a list of key terms starting with 'ear'. The system replaces the key term, after informing the user, by the preferred key term labyrinth. Now a window is opened showing in the centre a node labelled with the preferred key term. When we click on it, related key terms are added to the window, connected by a line indicating the type of relation. In this case the parts of the labyrinth are added. Double clicking on a key term displays a description of the key term, and so we decide to double click on Cochlea. Its description, shown below, makes us decide to have a look at its related key terms:

"The part of the internal ear that is concerned with hearing. It forms the anterior part of the labyrinth, is conical, and is placed almost horizontally anterior to the vestibule."

Now there are two relation types visible: part of and adjacent to. After reading the various descriptions, we realise that Spiral Ganglion is the key term we are looking for. It is adjacent to the cochlea and connected to the cochlear nerve.

We now have found the right key term; all we must do is finding the related documents. How they can be found is depicted in Figure 4.4. Starting from Spiral Ganglion, this screen is obtained after a few mouse clicks. We see three key terms that are subterms of Spiral Ganglion: Spiral Ganglion embryology, Spiral Ganglion metabolism, and Spiral Ganglion cytology. Each of these subterms has a sec keyterm 


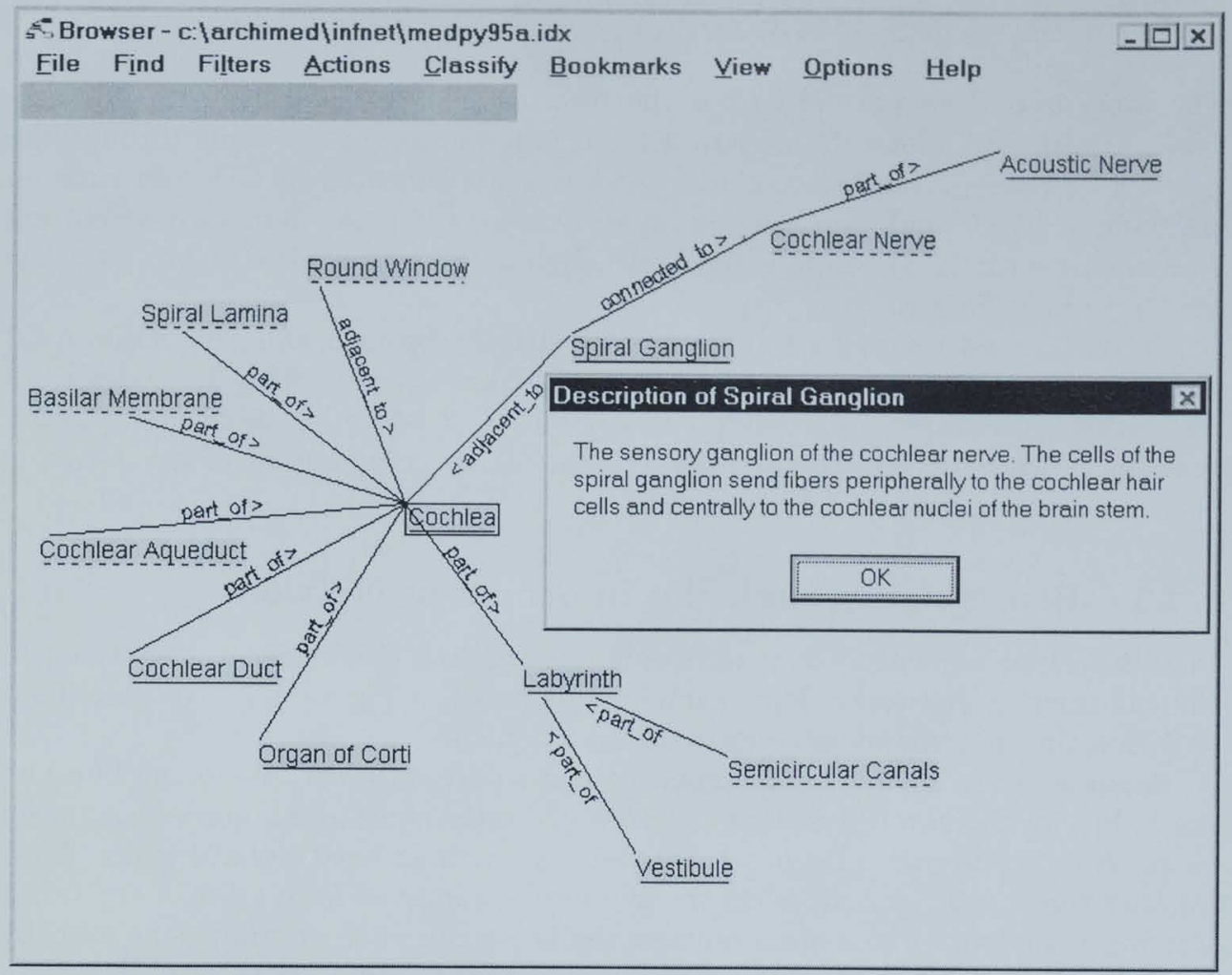

Figure 4.3: Screen dump of the browser showing medical concepts from the example.

relation with one or more document nodes. This relation expresses that the document in question is about the key term, but the key term is only of secondary importance for the document. The three subterms have no documents of primary importance linked to it. To see the other key terms related to a particular document we can of course click on the document, and the browser will make them visible with links connecting to the document. To see the content of a document we double click on it. The content of the document is then fetched and presented, as can be seen at the bottom of Figure 4.4.

In the example above, the document was a Medline record. Also hypermedia documents can be accessed with ANS. Hypermedia documents are commonly viewed with a hypermedia browser, which presents to the user a part of the hypermedia document, and allows him to follow hyperlinks to other parts of the document. The ANS browser is a meta-browser, in the sense that it does not directly show the contents of the document parts, but the relations between the document parts 


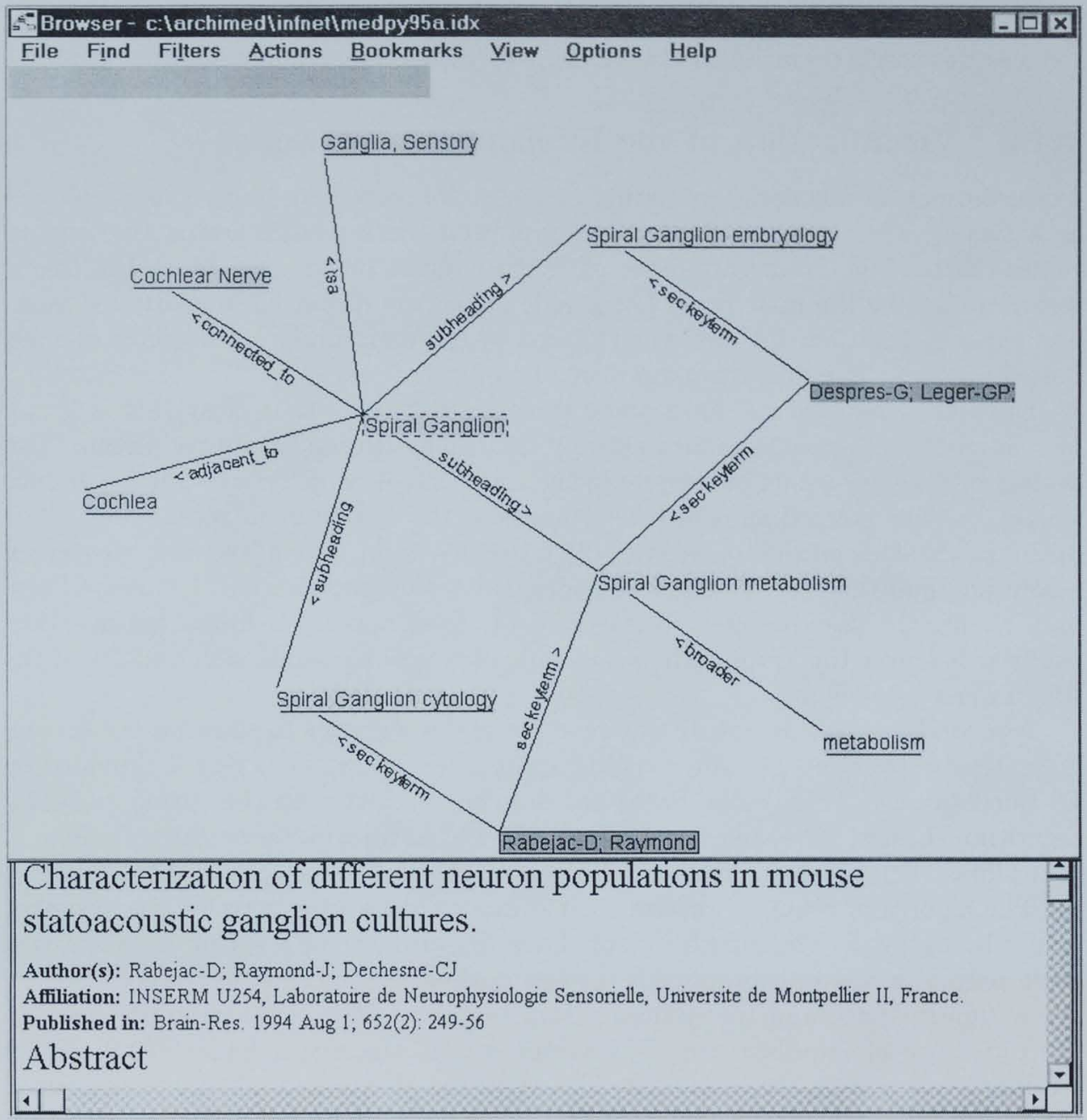

Figure 4.4: Screen dump of the browser showing medical concepts and documents from the example.

as well as the concepts the document parts contain. Actually to view a document part, the user can double click on it, and the DAP (in this case the hypermedia browser) will present it. As stated in Section 4.2.2, we tested this on a part of the Virtual Hospital. Since it is a part of the Web, we could speak of ANS as a 'metaWeb-browser'. Conklin and Begeman (1988), who describe a system that visualizes a hypertext, without concepts, give two advantages of hypertext visualization. Firstly, 
it reduces disorientation of the user by providing a sense of context. Secondly, it reduces the user's cognitive load because it serves as a mnemonic device.

\subsubsection{Visualization of the Information Network}

The Information Network, consisting of nodes connected by links, can be viewed as a graph. The system visualizes the graph on the screen, allowing the user to browse through it. Nodes are depicted by their name in a box of which the colour is determined by the node type. Long node names are displayed in shortened form, and expanded to their full size when clicked by the user. Links are depicted as lines between nodes. The link types are printed along the lines.

Drawing a large graph on a small screen is difficult. It is clear that a graph of non-trivial dimensions cannot entirely be displayed on a computer screen. The system only shows a part of the graph; the user controls which part. Our experience taught us that a maximum of fifteen nodes is the optimum in most cases. The optimum depends on the topology of the particular Information Network, the screen resolution, and the user. With more nodes, the screen becomes easily cluttered and as a result, the user becomes disoriented. A lower maximum limits the overview the user has over the graph. The problem is of course to decide which nodes of the graph must be shown.

A second problem is how to organize the nodes we want to show on the screen. An extensive overview of many existing approaches to graph drawing is provided by Di Battista et al. (1994). We based our drawing algorithm on the spring embedder algorithm (Eades, 1984) but it can also be viewed as a variation on the technique of multidimensional scaling (Schiffman et al., 1981).

The algorithm requires for each pair of nodes a distance between the two that would be optimal. The algorithm simulates the presence of a spring between each node pair with a length proportional to its optimal distance. A difference between the actual distance and the desired distance will result in a force proportional to the difference of the distances. The vector sum of the acting forces of all springs working on the node are computed. The resulting force vector then is transformed into a displacement vector of equal size and direction. The node is translated over the displacement vector. For all nodes a displacement is computed and performed. The process is repeated until all vectors are zero, that is, the simulated springs are in rest.

The algorithm is applied every time that nodes are added to or removed from the view. When nodes are added, the algorithm requires initial positions for them. Randomly chosen positions can be used, but the closer the initial positions match the final positions, the faster the algorithm will terminate. Therefore, when a node is clicked upon to add its neighbours to the view, these are positioned in a circle around the clicked node. This procedure has turned out to be a good heuristic for determining the initial node positions. 


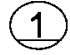

(a)

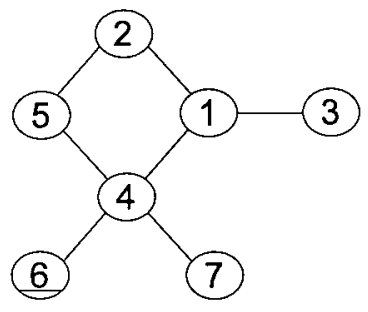

(c)

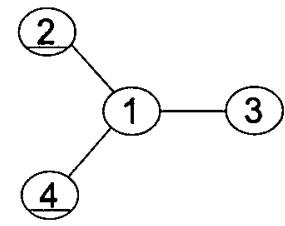

(b)

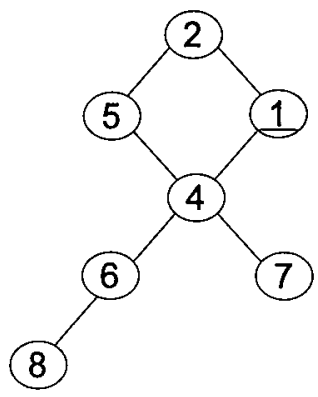

(d)

Figure 4.5: Four subsequent browser views of an Information Network.

For our purpose we would like to see a relation between the distance on the screen and the conceptual distance: two concepts that are directly related, should be nearer to each other on the screen than two concepts that are only remotely related. We defined the conceptual distance between two nodes as the length of the shortest path between the nodes. The algorithm tries to minimize in a series of iterations for each pair of nodes the difference between Euclidean distance and conceptual distance. No explicit checks are made for overlapping nodes on the screen. Since the algorithm strives to distances between nodes greater than 1 (because conceptual distance is always $\geq 1$ ) overlapping is avoided implicitly.

A problem arises for large Information Networks. To determine the conceptual distances in the graph, for every pair of nodes (in an Information Network of $n$ nodes: $n(n-1)$ pairs) the shortest path has to be determined. For one pair, in the worst case, the entire graph has to be searched. This process has to be repeated every time a change is made to the Information Network. We therefore decided only to take into account nodes that are on the display. This implies that distances have to be determined every time a node is deleted from or added to the display. Since the number of displayed nodes is small, this can be done very fast. 
The algorithm addresses the problem of which nodes of the graph to show by discarding nodes whenever more than the maximum allowed number of nodes is about to be shown. Assuming the user to be probably most interested in the node last clicked on, the nodes with the greatest conceptual distance to that node are discarded. Figure 4.5 shows schematically how the view of the Information Network changes. In this example the Information Network consists of eight nodes, and the maximum number of visible nodes is taken to be seven. A horizontal line inside a node indicates that the node has more neighbours than shown at that moment. The user starts with node 1 (a). After clicking on it, its neighbours are shown (b). After clicking on node 4 , the neighbours of 4 are added. Note that node 5 also has a link to node 2: when a node is added to the screen, any links to other nodes on the screen are drawn (c). When the user clicks on node 6 and its neighbour is added, the maximum number of visible nodes is exceeded, therefore one node has to be removed. Candidates are node 2 and node 3 , which have the greatest distance in the graph (i.e., greatest conceptual distance) to the newly added node 8 . Nodes 2 and 3 are equivalent, therefore the first one checked by the algorithm is removed (d).

If a node $\mathrm{N}$ has more neighbours than the maximum allowed number of nodes $m$, and the user clicks on it to see the neighbours, only $\mathrm{N}$ and its first $m-1$ neighbours are shown. When $\mathrm{N}$ is clicked upon again, the first $m-1$ neighbours are removed and the next $m-1$ neighbours are shown. This can be repeated until all neighbours have been shown; after that, the first neighbours are shown again. For large numbers of neighbours (compared to $m$ ) browsing through them has turned out to be tedious. Therefore two alternative ways of browsing through the neighbours of a node are provided: selection by link type and selection by name. The first way allows the user to choose from a list of the link types that are used to link $N$ to its neighbours. After selection, only the nodes of the selected link types are shown. This is, of course, effective only if the list contains more than one link type. The second way - selection by name - presents the user with a list of all neighbours in alphabetical order. The user can choose names from this list and then have them displayed as nodes.

Often, the picture drawn by the algorithm is quite nice. Sometimes it is not, for instance, when a line obscures a text or a text obscures a line. In that case the user can improve the picture by dragging nodes to better positions. This was actually done in Figure 4.3. In Figure 4.4 no changes were made. Since only a small number of nodes is involved by the drawing algorithm, it terminates fast. The user can browse through the Information Network in real-time on a 486 DX 66 machine.

Because the picture changes with every mouse click, the user may become confused. We solved this by showing the iterations on screen; thus the user sees how the new picture develops out of the old one. There is a trade-off between speed and clarity on one side and aesthetics on the other. The more iterations are made, the more time redrawing takes and the 'better' the picture becomes. On the other side, the more the picture changes the more confused the user may become. On a 
relatively slow machine (486 DX 66) we chose to stop the algorithm after a pre-set number of iterations. On a relatively fast machine (Pentium 90 ) we let the algorithm terminate.

\subsubsection{Navigation Aids}

When browsing through an Information Network of non-trivial size, one may easily become confused and disoriented. This problem also occurs in hypertext (Nielsen, 1990a). Several solutions used in hypertext systems can also be used in ANs. We discuss them below.

A bookmark facility enables the user to mark nodes of interest (key terms or documents). They are added to a bookmark list, from which later on in a session items may be selected for further browsing.

It often happens that the user wants to go a step backwards; a node was clicked, its neighbours were added but they were of no interest to the user. In order to add the neighbours, several other nodes were removed. In theory, it suffices to click the right node(s) to make them reappear. The user can see if he has clicked upon a node before, because the type faces of clicked and unclicked nodes are different. In practice however, the user often forgets which node he has to click on. This problem has been alleviated with the backtrack facility. It presents the user with exactly the situation of before the last mouse click. The user can backtrack ten steps in this way, and each of these steps can also be undone. The visualization algorithm is not used here: the system records node names and screen positions of the last ten situations. A previous version of ANS recorded only five situations, but several users complained that five was not enough. The ten steps in the current version are rarely used all.

If the user wants to go back any further, it is not because he remembers a particular situation, but rather a particular node name. In that case the history list can be used. It is a list of all node names clicked since the start of the session, in order of clicking. When a node name is selected from the list, it is added to the subgraph. If it is not linked to any of the other nodes on the screen, these are all removed. Thus, the history list does not record situations. The version of ANS described in Chapter 3 contained only the history list, not the backtrack facility. The latter was added because the informal evaluation (Section 3.5) showed that the history list alone was not sufficient.

The most important means to control what part of the Information Network is shown is the filter mechanism. It enables the user to filter out specific parts of the Information Network. With the link type filter one can specify what relations (link types) must be ignored when fetching the related nodes after a node has been clicked on. For instance, when the user is interested in anatomical concepts only (as in the example in Subsection 4.4.1), relations as manifestation of and causes can be ignored. With the node type filter one can specify what specific types of nodes must 
be ignored. For instance, when browsing through a multimedia book, the user may want to ignore documents of node type sound or X-ray.

Especially if an Information Network contains many link types and node types, selecting the right filters may be difficult. This was observed during the informal evaluation. To solve this problem we introduced filter pairs. The idea is that a particular stage in browsing corresponds with a particular node type filter and a particular link type filter. Stages may be for instance: browse documents only, browse main concepts only, ignore anatomical relations. For a particular Information Network several of these stages and corresponding node type - link type filter pairs can be predefined. Thus, the user does not have to deal with selecting the right filters anymore, but the decision is taken to a higher level: selecting a browsing stage. For the Medline Information Network seven browsing stages are distinguished: Show concepts; Show important related documents; Show all related documents; Show concepts \& subheadings (no documents); Show concepts, subheadings \& important related documents; Show concepts, subheadings \& all related documents; Show concepts and extra links (no documents). The stages are listed in order of restrictiveness. ${ }^{3}$ Lazy users do not select from the list but change to one stage lower or higher on the list than the current stage by issuing the commands 'Show more' or 'Show less'. Typically, the user starts with the most restrictive stage. During browsing one or more times a less restrictive stage is selected. Especially during backtracking the user selects a more restrictive stage.

When a more restrictive filter is chosen, it is possible that the graph becomes divided into several subgraphs (i.e., it is no longer a connected graph). Because the drawing algorithm cannot handle such a graph and the user is probably not interested in all subgraphs, the system discards all subgraphs but one. The subgraph containing the node that the user clicked last on is retained, which is probably the subgraph the user is most interested in. If that node was discarded as a result of the filter change, the subgraph is retained that contains the node the user clicked on before he clicked on the discarded node.

When browsing through the Information Network, it makes no sense to click on nodes from which all neighbours are already on the screen. Therefore the user needs to know which nodes on the screen have neighbours that are not on the screen. Otherwise he may have to click the nodes one by one. If a node has neighbours that are not shown, but will be shown if it is clicked on, the text in the node is underlined. If a node has neighbours that are not shown, and none of them will be when it would be clicked on because a filter prevents it, the text in the node is underlined with a dashed line.

Sometimes it is important to make a distinction between particular link types for filtering, but not for browsing. For instance, in our Medline Information Network we have the link types narrower, simpler, and related. For the first two, the user is not really interested in the names of these link types, only in the direction. Therefore

\footnotetext{
${ }^{3}$ The place in this list of the 'Show concepts and extra links (no documents)' stage is arbitrary.
} 


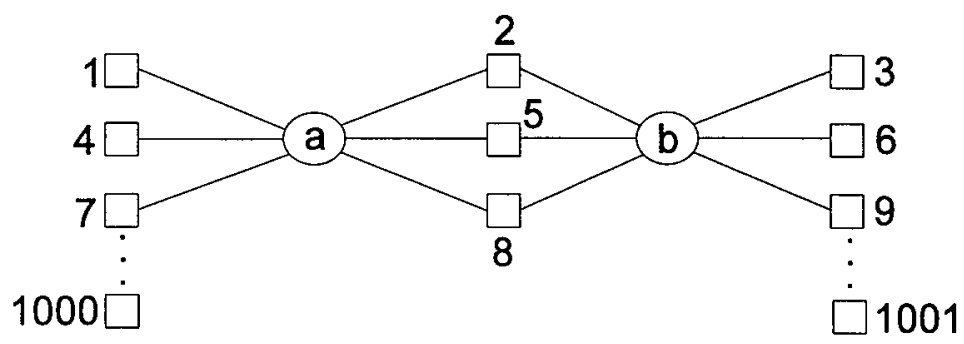

Figure 4.6: How to locate documents 2, 5, and 8?

the browser only draws the lines of these links and arrows to indicate the direction; the name of the link type is left out. In the case of the related link type also the direction arrow is left out as it is basically an undirected relation. In this way no superfluous information is presented to the user. In the current version of ANS it is not possible to specify which link types are to be handled like this; instead it is hard-wired in the program.

\subsubsection{Combination of Key Terms}

The browser mechanism described in the previous sections assumes that all relevant documents can be reached by one or more key terms. There are however cases in which this is a problem: a user will not be able to distinguish relevant documents from irrelevant ones. This happens if the user inspects a key term with an abundance of documents. If there is no other key term from which to 'approach' the documents, the user has no choice but to inspect every document (by looking at the other key words or at the document content). This is illustrated in Figure 4.6: assume documents 2, 5, and 8 are relevant. They are all linked to key terms a and $b$. The user is forced to browse either through all 502 documents linked to key term a or through all 502 documents linked to key term b. $^{4}$

This problem was solved by introducing an AND/OR-like facility which allows the user to make a combination of key terms and have a list of documents returned linked to these key terms. The user can compose two groups of key terms. Key terms within a group are oR-ed and between the groups an AND is performed. The user can inspect the documents retrieved as a result of the entire query or as a result of the group-1 part or group-2 part. The results can be inspected in two ways: as a list or as nodes in the browser. The list view shows a list of the documents and

\footnotetext{
${ }^{4}$ Even if a key term $\mathrm{c}$ would exist, linked only to documents 2,5 , and 8 , it could still happen that the user would not be aware of the existence of $c$ and therefore had to use key term $a$ and $b$ only.
} 


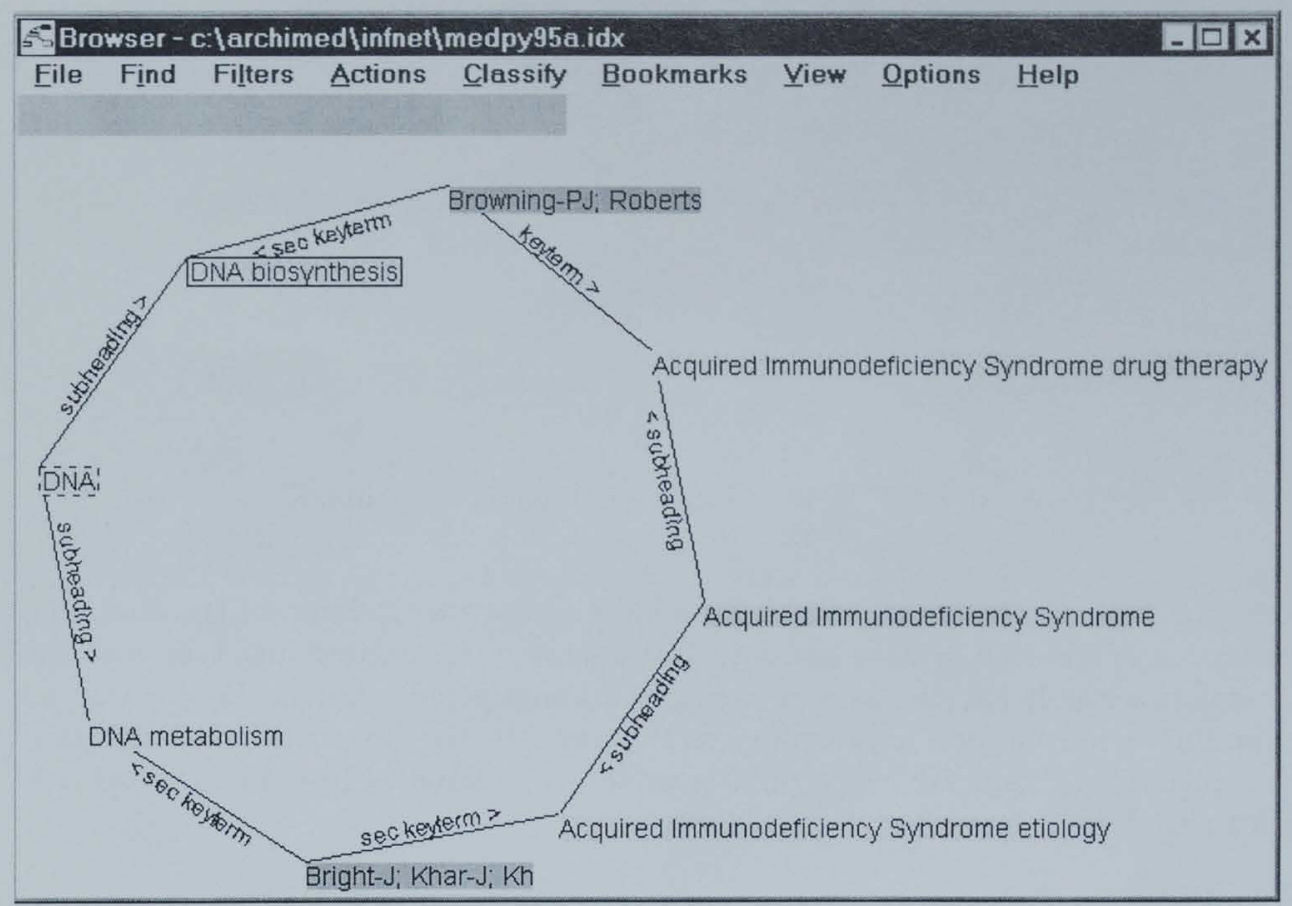

Figure 4.7: The result of combining AIDS with DNA.

their titles. Of course the user can bookmark documents and view their contents. The browser view depicts the documents and the key terms from the query as nodes in the browser window, thus offering the user new starting points for browsing. In the example above, the user can put key term a in one group and key term $b$ in the other group. The result of the combination is a list containing exactly documents 2 , 5 , and 8 . The two groups contain only one key term, therefore no or was performed. The following example illustrates a special use of the OR.

To find documents about AIDS and DNA the user selects AIDS into one group and DNA into the other. The system then finds the documents that are about both AIDS and DNA. Because of the use of subheadings only a few documents are linked to the main headings. In order to obtain the relevant documents concerning certain aspects of AIDS and DNA, the user should inspect the subheadings which actually reduce the scope of the main headings and are linked to the nodes containing the main headings. The OR-mechanism now allows the user to specify a number of relevant subheadings. If a key term that is linked to key terms of the node type mainsub is added to a group, the system shows these key terms corresponding to subheadings to the user and asks which of them should be included in the group. For instance, the key term AIDS has 
25 neighbours of type mainsub, the key term DNA has 19. If the user selects: AIDS etiology, AIDS blood, AIDS drug therapy, DNA blood, DNA biosynthesis, DNA metabolism, and DNA physiology, the resulting combination is equivalent with the Boolean query:

(AIDS OR AIDS etiology OR AIDS blood OR AIDS drug therapy) AND

(DNA blood OR DNA biosynthesis OR DNA metabolism OR DNA physiology)

Figure 4.7 depicts the browser view of this combination's results. Two documents were found; they are shown together with the key terms from groups 1 and 2 that are actually linked to these documents (because of the oR, not all key terms are necessarily used). The browser view of the combination results shows the user how the retrieved documents are related to the used key terms. This can be a valuable starting point for browsing. Often the number of documents retrieved together with the key terms from the query is too large to be displayed in the browser view. In that case the user must select a single document for displaying.

Because it only allows a conjunction of disjunctions of key terms the combination mechanism has less expressive power than the Boolean query language. This is not a shortcoming because the browser view easily lets the user find additional documents or discard unwanted ones. The advantage is a user interface that is easy to use.

\subsection{Chapter Conclusions}

In this chapter the shortcomings of the system that were identified in Chapter 3 have been dealt with. Not all features specified in Chapter 3 have been implemented: the underlying database and the retrieval of remotely stored documents is still limited. However, the database is only of concern to the administrator, not to the users; whether documents are stored locally or remotely is in ANS not relevant for the search. Therefore, we believe the second prototype of ANS as described in this chapter has been developed sufficiently far to be evaluated in a testing environment.

The diverse Information Networks constructed show the versatility of ANS. Especially for domains with a rich terminology, such as the medical domain, the ANS approach looks promising. 



\section{Chapter 5}

\section{Protocol for Evaluation}

In the two previous chapters, ANS was introduced. To find out how real users would perform with the system, an experiment was set up. This chapter describes the experimental design; the results are discussed in Chapter 6 .

Setting up an IR-system evaluation is not an easy task. There are many decisions to be taken, and many pitfalls to be avoided. Therefore, in Section 5.1 the literature on this subject is reviewed. Section 5.2 describes the resulting protocol: the goals of the experiment, the material, the operationalization, and the design.

\subsection{The Problem of Evaluating an IR System}

In this section we start with defining the IR evaluation terminology, then we discuss various aspects of an IR evaluation experiment: the kinds of evaluation experiments, measures for effectiveness and efficiency, the document set, the search questions, the retrieved set (i.e., the set of documents marked by a user as relevant), relevance, and the users.

\subsubsection{Terminology in IR System Evaluation}

The purpose of this section is to define the terminology that is used in our evaluation:

- The function of an IR system is to lead its users to those documents that will best enable them to satisfy their information need (Robertson, 1981).

- The effectiveness of an IR system is the extent to which the system carries out its function (Robertson, 1981).

- The efficiency of an IR system is at what cost the system functions (Robertson, 1981). 
- The information need of a user is a mental image that the user has of the information he wants to find in a session with an IR system.

- The question in an experiment is an assignment that a test person has to carry out (i.e., an artificial information need).

- The document set is the set of all the documents that the IR system can retrieve.

- The retrieved set is the set of documents that a particular user has found in answer to his information need.

\subsubsection{Designs of Evaluation Experiments}

A whole range of evaluation experiments exists. On the one end is the laboratory experiment, where the users, queries, documents, search constraints, and relevance assessments are completely controlled. On the other end is the operational experiment, where real users with real information needs search in real document sets. The advantage of the former is the absence of bias and the freedom of choosing the 'ingredients' (documents and queries) of the experiment. However, according to Oddy (1981) and Smithson (1994), laboratory experiments are likely to be misleading because real users with real information needs differ too much from artificial situations. The advantage of the laboratory experiment is that because more variables are controlled, observed results can be linked better to particular causes (Tague-Sutcliffe, 1992).

It is important to incorporate the user in the experiment, because, as Robertson (1981) argues, we want to find out whether the system satisfies the user's information need, not only whether it presents the right documents as answer to a formal command.

A second distinction that can be made is whether the experiment is a blackbox or a diagnostic experiment (Robertson, 1992). In a black-box experiment the experimenter only considers the inputs (the information needs) and the outputs (the retrieved sets). In a diagnostic experiment also the processes are studied that led to the output. Again, there is a range of possibilities. The black-box experiment suffices if it has to be decided which of two (or more) systems or principles under examination is the best. If the experimenter also wants to know why a system or principle is better, also information during the IR process must be obtained.

\subsubsection{Measures for Effectiveness and Efficiency}

A traditional comparison of IR systems involves (Robertson, 1992):

- A quantitative assessment of the relevance of question-document pairs

- A quantitative analysis of the different questions and the different documents 
- A qualitative assessment of which of the systems performs best

In Subsection 5.1.1 we defined the effectiveness of an IR system. This definition leaves the question how we can measure effectiveness. Robertson (1981) points out that first we have to choose what we want to measure. Do we want to measure how well the retrieved documents match the user's information need? Or to which extent the user says his information need is satisfied? Or the overall satisfaction of the user? Or something else? There is much debate in the IR community about this subject, but there is no definitive answer yet. The extent to which the retrieved documents match the user's information need is the most popular one, because it is suitable for several ways of formal analysis. Central to these measures is the concept of relevance. It is difficult to give a adequate definition that is also manageable. We elaborate on the subject of relevance in Section 5.1.7, but for the moment we assume that the relevance of any document can be determined unambiguously.

The most-widely-used measures for retrieval effectiveness are recall and precision (Kent et al., 1955). These measures were already introduced in Section 2.1, but we repeat them here in a more formal form:

$$
\begin{aligned}
\text { recall } & =\frac{\text { relevant documents in retrieved set }}{\text { relevant documents in document set }} \\
\text { precision } & =\frac{\text { relevant documents in retrieved set }}{\text { documents in retrieved set }}
\end{aligned}
$$

Recall and precision are determined for a particular test person. To determine recall and precision for a particular question, the averages are taken over all test persons who answered the question. To determine recall and precision for a particular system, the averages are taken over all questions. From the equation above follows that the number of relevant documents in the document set for a particular question must be known to determine recall. For document sets of non-trivial dimensions this is difficult. Taking a random sample and making an estimation using statistical techniques is not a solution, because of the probably small number of relevant documents in the document set. The problem can be avoided if two IR systems are compared. In that case, a relative recall can be measured by defining the relevant documents as the union of all relevant documents retrieved by at least one user (independent of the system used) (Tague-Sutcliffe, 1981).

A second problem is that recall and precision are two measures, and that a tradeoff exists between the two (Buckland and Gey, 1994): retrieving all documents of the document set, results in a recall of 1 and low precision. Likewise, retrieving only one - relevant - document, results in low recall and a precision of 1 . Thus, the one does not make sense without the other. For IR systems that produce ranked output it is possible to draw recall/precision curves by determining recall and precision at various cut-off values. For instance, one can take the 10, 20, and 30 highest ranked documents. The graph shows how for the particular system precision deteriorates as recall improves. 
Several attempts have been made to combine recall and precision into one measure (Shaw, 1986; Wilbur, 1992), but they are not used in practice. A measure that is used in practice is Rijsbergen's E-measure, or Rijsbergen's measure for short (Van Rijsbergen, 1979, Chapter 7). It is defined as:

$$
E=1-\frac{1}{\alpha\left(\frac{1}{P}\right)+(1-\alpha) \frac{1}{R}} \quad 0 \leq \alpha, P, R \leq 1
$$

where $P$ is precision, $R$ is recall and $\alpha$ is a constant that determines the importance of recall relative to precision. The value $\alpha=0.5$ indicates that recall and precision are considered equally important. A high value of $E$ means low retrieval effectiveness, and vice versa. Note that if $\alpha=0$ then $E=1-R$ and if $\alpha=1$ then $E=1-P$. Therefore, recall and precision can be considered as special cases of Rijsbergen's measure.

Su $(1992,1994)$ concludes from an experiment with mediated literature search (i.e., where a human acts as a mediator between the user and the IR system):

- The best measure for retrieval effectiveness is the value given by the user for the search results as a whole. ${ }^{1}$

- To users, recall is more important than precision.

- Precision is not a good indicator of success.

The efficiency of a system can be measured by the average time needed to complete a session or, if searching is not free, the average cost of a session.

From quantitative quality measures we now turn to qualitative quality measures. Qualitative assessment of the retrieval effectiveness can be done in the following ways:

- The user's actions during the session can be logged. Analysis of the logs may show how easy or difficult it was for the user to find the documents, and what strategies he used.

- Interviewing the user after finishing the session can give general information about the quality of the system.

- A thinking-aloud protocol of the searching user provides detailed information, but a well-known problem is that this influences the behaviour of the user.

\footnotetext{
${ }^{1}$ Users were asked to give a score on a seven-point scale.
} 


\subsubsection{The Document Set}

The document set is the set of documents that the IR system can retrieve. In Section 3.1.1 we defined a document as an amount of information declared by its producer to be a document. Apart from documents composed by real producers, also pseudo-documents, composed automatically, can be used. Pseudo-documents can be useful for specific search questions, but in general it is better to use real documents (Robertson, 1981).

An arbitrary set of documents can be chosen, or a test collection, which is a document set specially compiled for IR evaluation purposes. Several test collections are available, for instance the CACM collection, the Cranfield collection, the OHSUMED collection (Hersh et al., 1994), and the various TREC collections (Sparck Jones, 1995). A test collection consists of a document set, a set of search questions, and relevance rankings for the documents relative to the questions. Test collections of various sizes have been used. Shaw Jr. et al. (1997) give an overview of 17 test collections. The majority is small: 10 collections are smaller than 10,000 documents. 3 collections are in the range $11,000-27,000$. The 4 TREC collections in the overview are much larger: $173,000-742,000$ documents.

It is still unclear to what extent the performance of an IR system depends on the document set. There are at least strong indications that performance drops when the size of the document set grows, depending on the particular IR system. Therefore, conclusions obtained from experiments with one document set do not necessarily hold for larger document sets (see e.g., Blair and Maron, 1985).

The advantage of using a test collection is that the results of the evaluation can be compared with other systems that were evaluated with the same collection. However, if the IR system depends on a domain representation, then a representation of the domain of the collection may be difficult to acquire. For instance, ANS' Information Network contains a representation of the domain of the documents. Usage of the CACM test collection, which contains articles from Communications of the ACM, would require the representation of the computer-science domain in the Information Network.

\subsubsection{The Questions}

The user's information need may be fixed, though the user's perception of it may change during the session (Robertson, 1981). This is less likely to happen when the information need is artificial, that is, the information need is imposed by the researcher in the form of a question. A disadvantage of an artificial information need is that it is more difficult for the user to decide whether a particular document is important or not. According to Tague-Sutcliffe (1992) many researchers believe that real information needs are to be preferred.

In the case of artificial information needs it is on the one hand important that the questions are not formulated in a too detailed way, because that would almost 
answer the question; on the other hand the questions cannot be too vague: the test persons must all experience the same information need.

\subsubsection{The Retrieved Set}

The retrieved set is the set of documents that a particular user has retrieved in answer to his information need. It can be defined in various ways, depending on the IR system used. If results are not ranked, the entire result set (i.e., all documents retrieved by the query) constitutes the retrieved set. If results are ranked, the retrieved set can be obtained by cutting of the result set at a particular point. For instance, the first ten documents are taken. Another possibility is to let the user mark every document of the search results that he values as relevant.

\subsubsection{Relevance}

The recall and precision measures depend on the concept of 'relevance'. This is a central notion in IR. We limit ourselves to an overview of the points that directly pertain to IR-system evaluation. Mizzaro (1997) provides a general overview of the history of relevance in IR.

Several researchers question the way relevance of documents is dealt with in IRsystem evaluation. Saracevic (1995) claims that relevance depends on many variables like circumstances, context, and so on, and therefore is not a valid basis for evaluating IR systems. Also Harter (1996) questions the use of relevance assessments. He points out that reported variations in relevance assessment have a yet unknown effect on recall and precision. However, no solutions for these problems are given.

Who is to make the relevance assessments? It can be done by the users; Rees (1966) claims that if a user says a document is relevant, then it is relevant at that time and in that context. The problem is that to determine the recall, each user must assess the relevance of each document. Robertson (1981) and many other researchers prefer an assessment by a third party, a judge. Assessment of the documents by one judge may result in a bias caused by personal characteristics (such as preferences and knowledge gaps). To minimize the bias, multiple judges can assess the documents after which for each document a final relevance assessment can be determined.

A third option is to deduce the relevance of a document, for instance, by determining the frequencies in the documents of the key terms from the information need. The advantage of this approach is that it is not influenced by inconsistent human beings. However, the problem with this approach is that the algorithm that does the assessments must have knowledge of the documents' domain in order to take complexities into account as synonyms, homonyms, broader and narrower terms.

Belkin (1981) calls relevance as measured by the user situational relevance and deduced relevance logical relevance. Relevance as assessed by judges is merely called relevance. 
It may be expected that the judgement of a particular document is influenced by the documents the judge has assessed before (Robertson, 1981). Moreover, if judges are used and two systems are compared, the output of the systems should be mixed together. Usually the judge can choose from several categories (very relevant, relevant, etc.) which are then merged in either relevant or not relevant. This is because it is easier for a judge to choose from more than two categories (Robertson, 1981).

\subsubsection{The Users}

Tague-Sutcliffe (1992) states that many variables concerning the user in an evaluation are categorical. For instance: type of user (student, scientist), context of use (educational, research), kind of information need (aid in understanding a problem, define a problem, background reading).

The performance of the users may be influenced by the presence of the experimenter; this is called the Hawthorne effect (Roethlisberger and Dickson, 1939).

\subsubsection{Conclusion on IR System Evaluation Literature}

The previous sections described possibilities for setting up an IR system evaluation. We conclude that where for some issues the right choice is obvious, other issues are still unclear in the field of IR and require arbitrary decisions. The conclusion of Robertson in his 1981 article is still valid: "There is no such thing as a watertight method for evaluating an information retrieval system. There is, on the other hand, a considerable battery of methods and techniques for dealing with the various problems that arise in this endeavour" (Robertson, 1981, p. 30). In the next section, which presents our evaluation protocol, our selection from this battery will be discussed.

\subsection{Protocol}

Our starting points were:

- We did not want to evaluate a toy problem: the document set and the domain representation had to be comparable with a real situation.

- As this research was carried out at the Department of Medical Informatics, medicine was the preferred domain.

- Ans was to be compared with another IR system, because absolute effectiveness and efficiency values cannot be compared across experiments.

An additional advantage of the last point is that it alleviates the problem of determining the relevant set: only retrieved documents are taken into account.

With these starting points in mind, several choices mentioned in Section 5.1 can be decided upon. 


\subsubsection{Objective and Features of the Experiment}

The objective of the experiment was to study the difference in performance of a system like ANS and a standard Boolean IR system. We define performance as the combination of effectiveness, efficiency, and user satisfaction.

The experiment was executed as follows. A number of test persons was asked questions about the medical domain. The questions were of the form "Find documents about..."; the test persons had to answer them using two IR systems. In Section 1.5 we stated that the system will be beneficial especially for users with little domain knowledge. Therefore, test persons were divided into two groups:

1. Test persons with relatively little domain knowledge

2. Test persons with relatively much domain knowledge

With 'domain knowledge' we mean knowledge of the medical domain in general and detailed knowledge of the subjects of the questions. The test persons were recruited under medical students. Students who were in their second year of medical school were assigned to group 1, students who were in their fourth year were assigned to group 2. Domain experts formulated the questions, which were to be answered by all test persons. Each test person answered one question with ANS, and two with the Boolean system, or vice versa. The result of a question for a test person was a set of documents. For each question the union of the results was determined. For each union two domain experts judged the relevance of each document. From these relevance judgements a measure for effectiveness could be determined. The efficiency of a system was measured by the average search time. To establish the user satisfaction, for each question the test persons were asked how satisfied they were with the result. Finally the test persons were interviewed about their experiences with the systems.

The general features of the experiment are:

Independent variable: the IR system

Dependent variables: effectiveness, efficiency, user satisfaction

Unit of analysis: the test person

Disturbing variables: domain knowledge of the test person and the expert, question, searching experience of the test person, meticulousness of the test person, learning effect for the test person and expert

Hypotheses: see Section 5.2 .3 


\subsubsection{Material}

\section{Questions}

The questions that the test persons had to answer were formulated by experts in the medical domain, in cooperation with the researcher. The questions concerned airways disorders, fractures, and eclampsia. The appendix discusses them in detail.

\section{Documents}

For the document set we considered two possibilities: using the Medline biomedical literature database, which contains document abstracts, or creating a full-text document set from medical textbooks. We chose the former because it relieved us from scanning and indexing the documents (Medline records already contain index terms).

Our prototype was not capable of including all the millions of records of Medline. ${ }^{2}$ Therefore a subset was used: the Silverplatter Medline Express CD-ROM of JanuaryFebruary 1995, which contains 35,964 records.

Since we chose to include the records that were added in a particular period, we assume that the selection is a homogeneous, representative subset of the entire database.

\section{Research Instruments}

As research instruments two different IR systems are used: ANS and WinSpirs 2.0. ANs was discussed in the previous chapter, and WinSpirs is one of the systems looked at in Section 2.3. It is the MS windows version of Spirs, the standard IR system for Medline in our university library. It is a common misconception that Medline is an IR system. However, Medline is a literature database and various IR systems exist to search in it. One of them is WinSpirs; other examples are Grateful Med, Knowledge Finder, and PaperChase.

WinSpirs (also erroneously called Silverplatter) is a Boolean search system with a thesaurus containing MesH terms (Medical Subject Headings). MesH terms are a subset of UMLS, which is the basis of ANS' Information Network. Each record of the CD-ROM contains MesH key terms, that represent the content of the document. Both ANS and WinSpirs use these key terms. Thus, we do not introduce a bias by using different indexes. In addition to conceptual retrieval, WinSpirs can also search full-text on the record fields (which includes the abstract).

\footnotetext{
${ }^{2}$ This is merely a limitation of the database used in the current implementation. At the end of 1997, Medline contained 9.6 million documents.
} 


\subsubsection{Operationalization}

The central question in this experiment was: are effectiveness, efficiency, and user satisfaction of the information retrieval process better with ANS than with WinSpirs? This question was operationalized in four hypotheses:

1. ANS is more effective than WinSpirs.

Effectiveness of an IR system is expressed in recall and precision, as defined in Subsection 5.1.3. We expected a higher recall for ANs because it is easier to find the relevant concepts and consequently the relevant documents. We expected a higher precision, because it is easier to distinguish between relevant and irrelevant concepts and consequently between relevant and irrelevant documents. Consequently, we expect a lower value for Rijsbergen's measure.

2. ANS is less efficient than WinSpirs.

Efficiency of an IR system is expressed in the time used by the users to find the results. We do not expect ANS to be more efficient. Because of its more complicated and extensive interface it takes more time to become familiar with ANS than with WinSpirs. The test persons had only a limited time to practice with the systems. After a longer use we expect users to be more efficient with ANS. However, we did not investigate this. Furthermore, the browsing character of ANS search may also be a cause for lower efficiency: in an experiment Waterworth and Chignell (1991) found that query-oriented search was more efficient than browse-oriented search.

3. The test persons are more satisfied with ANS than with WinSpirs.

User satisfaction of an IR system is determined by asking the test persons for a rating (from 1 to 5 , both inclusive) of their result for a question.

4. The advantage of ANS over WinSpirs in terms of effectiveness, efficiency, and user satisfaction is greater for group 1 than for group 2.

We expected the test persons of group 1, who had little domain knowledge, would benefit more from ANS than the test persons of group 2, who had more domain knowledge.

\subsubsection{Design}

This subsection describes the design of the experiment. It discusses the unit of analysis, the possible biases, the samples, the experimental set-up, and the relevance judgements.

\section{Unit of Analysis}

Potential test persons are second-year and fourth-year medical students, in compliance with the following restrictions. Excluded from the experiment are students who: 
(1) have no experience with MS windows, (2) have much experience with (Win)Spirs or ANS, or (3) have participated in the pre-test of this experiment.

For group 1 also excluded are students who followed several medical courses in a previous study, for instance biological health sciences. Students who followed only a few medical courses cause no problems, because that does not add much to two years of medicine studies.

The persons were recruited with posters and by giving short presentations during regular lectures. All candidates that complied with the restrictions were accepted, until the required number of persons was obtained.

\section{Biases}

It can be expected that various variables can influence the results of the experiment. To assess the disturbance and to control it where possible, the following precautions were taken.

Computer skills of the test person Differences in mouse skills of the test persons can be disadvantageous for ANS. As a condition for participation in the experiment some experience with MS windows was required.

Medical knowledge of the test person Within a group, the test persons need to have roughly the same medical knowledge. During their studies all students follow the same courses. Students who followed too many medical courses during previous studies were excluded from the experiment.

Experience of the test person with the IR system Test persons were asked about their experience with IR systems. Those with too much experience were excluded from the experiment.

Learning effect of the test person To neutralize the effect, the systems were used in varying order.

Expertise of the domain expert As domain expert a clinician was chosen who was specialized in the subject of the question. For each question, two domain experts were selected and their relevance assessments were combined, resulting in a balanced assessment.

Learning effect of the domain expert The documents were judged in such an order that documents that were 'easy' to find and documents that were 'difficult' to find were mixed. Furthermore, the two experts judged the documents in different orders.

\section{Sample}

To limit the time required from experts and test persons, three subjects were chosen, with one question per subject. For each of the questions a search had to be conducted 
with both IR systems, which means there are $3 \times 2=6$ possible combinations of a question and a system.

To vary the order in which the questions are answered leads to $3 ! \times 6=36$ possibilities. For two groups this would imply a total of 72 test persons. Since this number was not feasible, a choice had to be made between not varying the order of the questions or having 2 instead of 3 questions. We assumed that using only 2 questions would make the experiment too dependent on the choice of the question topics. Since the measurement of the learning effect of the test persons and the relative difficulty of the questions is of secondary importance, the first option is chosen.

In Table 5.1 an overview is given of a possible arrangement of the IR systems over the test persons and questions.

Table 5.1: Arrangement of the IR systems over test persons and questions.

\begin{tabular}{lccc}
\hline & \multicolumn{3}{c}{ Question } \\
\cline { 2 - 4 } Test person & Fractures & Obstetrics & Airways disorders \\
\hline 1 & ANS & WinSpirs & ANS \\
2 & ANS & ANs & WinSpirs \\
3 & WinSpirs & ANs & ANS \\
4 & WinSpirs & WinSpirs & ANS \\
5 & ANS & WinSpirs & WinSpirs \\
6 & WinSpirs & ANs & WinSpirs \\
\hline
\end{tabular}

We determined the probability that a difference would be found if a difference existed (i.e., the power of the test). We assume that the difference must be at least 1 standard deviation. From Schouten (1995, p. 202) follows that the power of the test - with a $2 / 1$ cross-over trial - is 0.99 when 24 test persons are used (i.e., for the two groups together).

\section{Experimental Set-up}

The test persons were subjected to the test one at a time so that they could not influence each other. Thus the experimenter was sure that all questions of a test person concerning the operation of the systems could be answered by the experimenter and that test persons did not crib. This was considered more important than avoiding a possible Hawthorne effect (discussed in Subsection 5.1.8).

At the beginning of a session the working of the IR systems was explained. Next, the test person answered a practice question with both systems (one question for each system), which was not taken into account in the analysis. The experimenter would answer any of the person's questions. For the three real questions, the search 
Table 5.2: Conversion of expert judgements to final relevance.

\begin{tabular}{ccc}
\hline Experts' judgements & Final relevance \\
\hline 1 & 1 & irrelevant \\
1 & 2 & irrelevant \\
1 & 3 & irrelevant \\
1 & 4 & $?$ \\
2 & 2 & irrelevant \\
2 & 3 & $?$ \\
2 & 4 & relevant \\
3 & 3 & relevant \\
3 & 4 & relevant \\
4 & 4 & relevant \\
\hline
\end{tabular}

results were filed. After each question, the test person was asked to assess the course and the results of the search (on an ordinal scale from 1 to 5 , both inclusive). The maximum time allowed for a question was 20 minutes. A pre-test with 5 test persons indicated this was a reasonable time. When the time had passed, the results found up to that moment were taken as the final result. All actions of the test person were logged with a time stamp. At the end of the session the test person was asked not to discuss the questions with (potential) future test persons.

Because neither of the two systems provides ranked output, the retrieved set was defined as the documents that were marked by the user as relevant (as discussed in Subsection 5.1.6).

\section{Relevance Judgements}

In Subsection 5.1.7 the concept of relevance was discussed. We followed the approach of the majority of IR experiments: judges assess the relevance of the documents.

After the sessions with the test persons, the experts judged the relevance of the retrieved documents. They were given the same information on each document as the test persons: title, names and affiliations of the authors, bibliographic information, abstract (if present), and MeSH terms.

For each question the judgements were made by two experts; one of them had formulated the question. For each document the expert judged the relevance of the document in relation to the question on a 4-point Likert scale: 1: very irrelevant, 2: irrelevant, 3: relevant, 4: very relevant. The first expert judged the documents in alphabetical order of the authors (which is as good as random order), the second judged in reverse alphabetical order. The experimenter commented on the first few relevance judgements as to make sure the expert had a proper conception of his task. 
Table 5.2 shows how the relevance judgements of two experts were combined in one final judgement. The judgements 14 and 23 were handled in a special way.

For each question the two experts were asked to comment on the cases where their judgements differed. In several of these cases the final judgement could be made. The remaining cases were all 23 judgements. Randomly, one half was assigned 'relevant' the other half 'irrelevant'.

\footnotetext{
${ }^{3}$ In case of an odd number, one randomly chosen case was randomly judged 'relevant' or 'irrelevant'.
} 


\section{Chapter 6}

\section{Evaluation Results}

In Chapter 5 the design of an experiment for the evaluation of ANS was presented. In this chapter the experiment's results are discussed. In Section 6.1 the judgements of the experts are compared. In Section 6.2 the scores of ANS and WinSpirs are presented and compared. Section 6.3 analyses the searches that were conducted. To what extent the test persons actually made use of the two IR systems is discussed in Sections 6.4 and 6.5. The results of the interviews held after each session are presented in Section 6.6. Finally, Section 6.7 presents the conclusions of the evaluation.

\subsection{Comparison of the Relevance Judgements}

As described in the Subsection 5.2.4, all documents that were found by at least one test person were subjected to relevance judgements by experts. For each of the three questions two experts independently judged the relevance of each document on a 4point Likert scale (1: very irrelevant, 2: irrelevant, 3: relevant, 4: very relevant). First we determined the agreement between the experts.

Tables 6.1, 6.2 and 6.3 show the agreement of the experts for questions A, B, and C, respectively. 'Expert A1' denotes the first expert for question A, 'Expert A2' denotes the second expert for question $A$, and so on. From these tables the absolute agreement and the kappa value can be determined. The absolute agreement is shown in the second column of Table 6.4. The kappa value is a measure for the agreement between two experts. It ranges from -1 (total disagreement) to 1 (total agreement). In the fourth column of Table 6.4 the kappas are shown as they follow from the previous tables. Landis and Koch (1977) provide a characterization of kappa ranges: values larger than 0.75 may be interpreted as excellent agreement, values between 0.40 and 0.75 as fair to good agreement, and values below 0.40 as poor agreement. Hence we concluded that the values we found, ranging from 0.40 
Table 6.1: Comparison of experts for question A on a 4-point Likert scale.

\begin{tabular}{|c|c|c|c|c|c|c|c|}
\hline \multirow{7}{*}{$\begin{array}{c}\text { Expert } \\
\text { A1 }\end{array}$} & \multirow[b]{3}{*}{1} & \multicolumn{4}{|c|}{ Expert A2 } & \multirow{2}{*}{\multicolumn{2}{|c|}{ Total }} \\
\hline & & 1 & 2 & 3 & 4 & & \\
\hline & & 12 & 2 & 3 & 1 & \multirow{2}{*}{$\begin{array}{r}18 \\
9\end{array}$} & \multirow{2}{*}{$\begin{array}{l}(34.6 \%) \\
(17.3 \%)\end{array}$} \\
\hline & 2 & 2 & 1 & 6 & & & \\
\hline & \multirow{2}{*}{$\begin{array}{l}3 \\
4\end{array}$} & & 1 & 5 & 5 & 11 & \multirow{2}{*}{$\begin{array}{l}(21.2 \%) \\
(26.9 \%)\end{array}$} \\
\hline & & & & 3 & 11 & 14 & \\
\hline & Total & $\begin{array}{r}14 \\
(26.9 \%)\end{array}$ & $\begin{array}{r}4 \\
(7.7 \%)\end{array}$ & $\begin{array}{r}17 \\
(32.7 \%)\end{array}$ & $\begin{array}{r}17 \\
(32.7 \%)\end{array}$ & 52 & $(100 \%)$ \\
\hline
\end{tabular}

Table 6.2: Comparison of experts for question B on a 4-point Likert scale.

\begin{tabular}{|c|c|c|c|c|c|c|c|}
\hline \multirow{5}{*}{$\begin{array}{c}\text { Expert } \\
\text { B1 }\end{array}$} & \multirow[b]{3}{*}{1} & \multicolumn{4}{|c|}{ Expert B2 } & \multirow{2}{*}{\multicolumn{2}{|c|}{ Total }} \\
\hline & & 1 & 2 & 3 & 4 & & \\
\hline & & 31 & 10 & & 1 & 42 & \multirow{2}{*}{$\begin{array}{l}(25.5 \%) \\
(30.9 \%)\end{array}$} \\
\hline & 2 & 6 & 21 & 9 & 15 & 51 & \\
\hline & 3 & & 2 & 7 & 18 & 27 & $(16.4 \%)$ \\
\hline & 4 & & 2 & 4 & 39 & 45 & $(27.3 \%)$ \\
\hline \multicolumn{2}{|r|}{ Total } & $\begin{array}{r}37 \\
(22.4 \%)\end{array}$ & $\begin{array}{r}35 \\
(21.2 \%)\end{array}$ & $\begin{array}{r}20 \\
(12.1 \%)\end{array}$ & $\begin{array}{r}73 \\
(44.2 \%)\end{array}$ & 165 & $(100 \%)$ \\
\hline
\end{tabular}

Table 6.3: Comparison of experts for question $\mathrm{C}$ on a 4-point Likert scale.

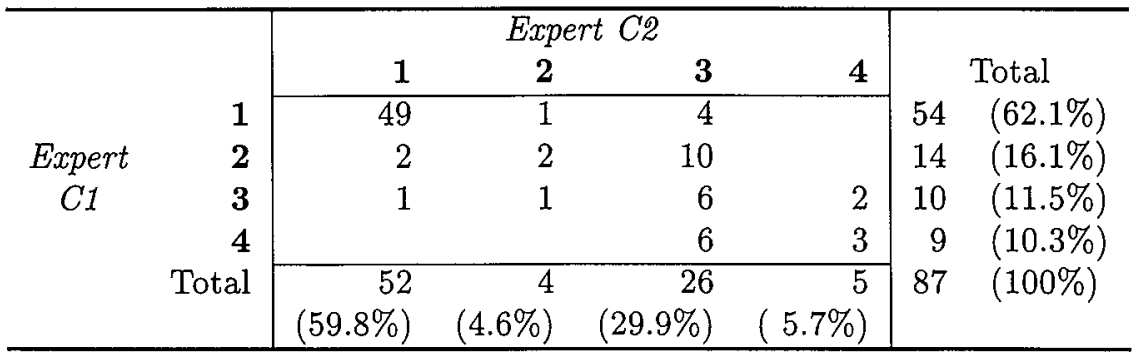


to 0.47 , correspond with fair to good agreement. A well-known cause for less-thanexcellent agreement are the different opinions of the distinct experts on where to put the boundary between relevant and irrelevant. Additionally, different experts may have different interpretations of the search question. The experimenter tried to prevent these biases by giving verbose instructions and feedback on the first few documents that were judged. Another cause for less-than-excellent agreement was that the experts, although this was not in line with the instructions, sometimes took into account factors such as the journal in which the document was published, the authors or their affiliations, and whether the particular subject of the document was of professional interest to the expert. Especially expert C2 was corrected several times on these issues.

We mapped the 4-point scale on a 2-point scale (1: irrelevant, 2: relevant) using the procedure described in Subsection 5.2.4 (see Table 5.2). After all, the only reason to use a 4-point scale instead of a 2-point scale was, as mentioned in Subsection 5.1.7, to make the choice easier for the expert. The results are shown in Tables 6.5, 6.6, and 6.7: they show the agreement of the experts for the 2-point scale judgements. The corresponding kappa values are shown in the last column of Table 6.4. These values correspond with fair to good agreement.

\subsection{Comparison of the Systems}

This section assesses ANS and WinSpirs by comparing the various variables that were measured. Subsection 6.2.1 describes the method of analysis used and Subsections 6.2.2 through 6.2.6 present the results of the analysis. Subsection 6.2.7 discusses an extension to the experimental design that was carried out.

\subsubsection{Method of Analysis}

The variables that resulted from the experiment were recall, precision, Rijsbergen's measure $(\alpha=0.5)$, time used to complete the search question, and user satisfaction. They were discussed in Subsection 5.1.3. To determine to what extent the variance in a variable was caused by controlled factors of the experiment an analysis of variance (ANOVA) was carried out. The factors used for the ANOVA were: test person, question, system, and group (second-year or fourth-year students). For the first three factors the main effects were determined. This was not possible for the factor group: because a test person is associated with a group, the factor test person already explains the variance caused by the factor group. The interactions ${ }^{1}$ between question and system and between group and system (notation: question * system and group * system, respectively) were also part of the ANOVA model. No interactions with test person were examined because we were not interested in the results of individuals. The

\footnotetext{
${ }^{1}$ An interaction is a source of variance that cannot be attributed to a single factor but only to a combination of factors.
} 
Table 6.4: Absolute agreement and kappa value for the various questions and scales.

\begin{tabular}{|c|c|c|c|c|}
\hline \multirow[b]{2}{*}{ Question } & \multicolumn{2}{|c|}{ Absolute agreement } & \multicolumn{2}{|c|}{ Kappa } \\
\hline & 4-point & 2-point & 4-point & 2-point \\
\hline$\overline{\mathrm{A}}$ & 0.56 & 0.79 & 0.40 & 0.58 \\
\hline B & 0.59 & 0.82 & 0.45 & 0.65 \\
\hline $\mathrm{C}$ & 0.69 & 0.82 & 0.47 & 0.56 \\
\hline
\end{tabular}

Table 6.5: Comparison of experts for question A on a 2-point Likert scale.

\begin{tabular}{|c|c|c|c|c|c|}
\hline \multirow[b]{3}{*}{ Expert } & \multirow[b]{3}{*}{1} & \multicolumn{2}{|c|}{ Expert A2 } & \multirow{2}{*}{\multicolumn{2}{|c|}{ Total }} \\
\hline & & 1 & 2 & & \\
\hline & & 17 & 10 & 27 & $(51.9 \%)$ \\
\hline$A 1$ & 2 & 1 & 24 & 25 & $(48.1 \%)$ \\
\hline & Total & $\begin{array}{r}18 \\
(34.6 \%)\end{array}$ & $\begin{array}{r}34 \\
(65.4 \%)\end{array}$ & 52 & $(100 \%)$ \\
\hline
\end{tabular}

Table 6.6: Comparison of experts for question B on a 2-point Likert scale.

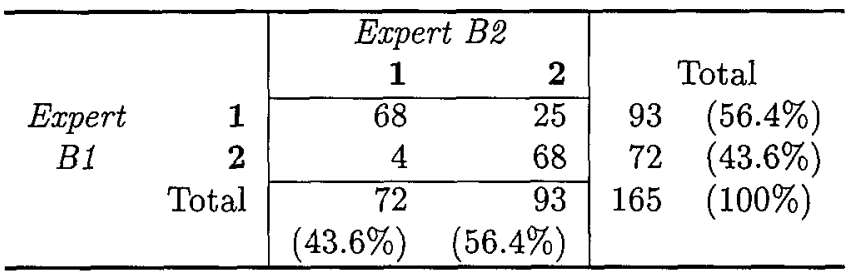

Table 6.7: Comparison of experts for question $\mathrm{C}$ on a 2-point Likert scale.

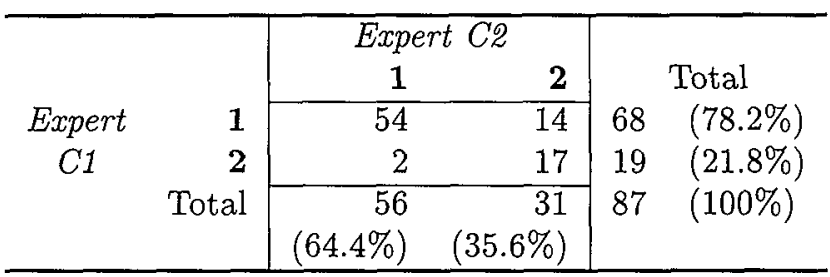


table that results of an ANOVA shows for each possible source of variance its sum of squares, the degrees of freedom, the mean sums of squares, the F-ratio (which indicates how much of the total variance is explained by the particular source of variance), and a p-value (which is the significance of the F-ratio).

The application of ANOVA requires that the data are normally distributed. This is more or less the case for the various variables, except for time used. The cause was that in $35 \%$ of the cases the maximum time allowed was used. However, the ANOVA residuals turned out to be normally distributed. ANOVA is known to be rather lenient when it comes to the normality condition. So as to determine whether there were cases with an excessive influence on the ANOVA for all cases for all variables Cook's distance was computed. This distance measures how much the ANOVA's estimated coefficients shift (relative to the standard error of those coefficients) when a data point is omitted. ${ }^{2}$ In one case a large distance was found; this is reported in the corresponding Subsection (6.2.7).

Following the ANOvA table the mean values of the particular variables are shown for ANS and WinSpirs. The differences between ANS and WinSpirs were compared with a least-significant difference pairwise multiple comparison test (LSD test). This test is equivalent to performing multiple $T$ tests of all pairs. The differences between groups were also compared with an LSD test.

\subsubsection{Recall}

Table 6.8 shows the ANOVA results for the variable recall, the fraction of the relevant documents that a test person found. The factor test person was the only significant source of variance: not surprisingly, the recall depended on the person who conducted the search.

The interaction between question and system was not significant $(p=0.154)$. There was a significant difference $(p=0.042)$ between the two systems for group 1 (the second-year students), but not for group 2. The differences are shown in Table 6.9. ANs scored a higher recall than WinSpirs for group 1. The recall for ANS is the same for both groups (according to Table 6.10), but the recall for WinSpirs is higher for group 2 than for group 1. Table 6.10 shows that this difference is significant $(p=0.001)$. Apparently, group 1 benefited more from ANS' domain knowledge than group 2. The difference in recall between the groups can also be attributed to the difference in IR experience between the groups. The overall mean recall was 0.152 .

As for the effect of the questions on recall, it was significant neither as main effect, nor as interaction with the factor system (Table 6.8). The results for the individual questions, in Table 6.9, show that for question B ANS had a significantly higher recall than WinSpirs. The difference corresponds with an average of 17 documents found with ANS and 10 with WinSpirs. For the other two questions no significant differences were found.

\footnotetext{
${ }^{2}$ An outlier does not necessarily have a strong influence on the coefficients, a case with a large Cook's distance does.
} 
Table 6.8: ANova test for recall.

\begin{tabular}{lcrccc}
\hline Source of variance & SS & DF & MS & F & p \\
\hline Test person & 0.434 & 23 & 0.019 & 2.220 & 0.012 \\
System & 0.001 & 1 & 0.001 & 0.132 & 0.718 \\
Question & 0.037 & 2 & 0.019 & 2.194 & 0.124 \\
Question * system & 0.033 & 2 & 0.017 & 1.958 & 0.154 \\
Group * system & 0.028 & 1 & 0.028 & 3.348 & 0.074 \\
individual error & 0.357 & 42 & 0.009 & & \\
\hline
\end{tabular}

Legend: SS: sum of squares; DF: degrees of freedom; MS: mean sums of squares; F:F-ratio; p: significance of $F$.

Table 6.9: Recall per group and per question.

\begin{tabular}{lccccc}
\hline & & \multicolumn{2}{c}{ Recall } & & \\
\cline { 3 - 4 } & & ANS & WinSpirs & Difference & p-value \\
\hline Group & & & & & \\
& 1 & 0.154 & 0.089 & 0.065 & 0.042 \\
Question & 2 & 0.163 & 0.203 & -0.040 & 0.208 \\
& & & & & \\
& A & 0.113 & 0.140 & -0.027 & 0.481 \\
& B & 0.188 & 0.108 & 0.080 & 0.040 \\
& C & 0.174 & 0.189 & -0.015 & 0.689 \\
\hline
\end{tabular}

Table 6.10: Differences in recall between the groups.

\begin{tabular}{lcc}
\hline System & Group 1 - Group 2 & p-value \\
\hline ANS & -0.010 & 0.751 \\
WinSpirs & -0.114 & 0.001 \\
\hline
\end{tabular}


Table 6.11: ANova test for precision.

\begin{tabular}{lcrccc}
\hline Source of variance & SS & DF & MS & F & p \\
\hline Test person & 2.917 & 23 & 0.127 & 1.896 & 0.035 \\
System & 0.027 & 1 & 0.027 & 0.407 & 0.527 \\
Question & 0.394 & 2 & 0.197 & 2.946 & 0.064 \\
Question * system & 0.013 & 2 & 0.063 & 0.094 & 0.911 \\
Group * system & 0.011 & 1 & 0.011 & 0.165 & 0.686 \\
individual error & 2.810 & 42 & 0.067 & &
\end{tabular}

Legend: SS: sum of squares; DF: degrees of freedom; MS: mean sums of squares; F:F-ratio; p: significance of $F$.

Table 6.12: Precision per group and per question.

\begin{tabular}{lccccc}
\hline & & \multicolumn{2}{c}{ Precision } & & \\
\cline { 3 - 4 } & & ANS & WinSpirs & Difference & p-value \\
\hline Group & 1 & 0.704 & 0.780 & -0.076 & 0.383 \\
& 2 & 0.816 & 0.805 & 0.011 & 0.890 \\
Question & & & & & \\
& A & 0.833 & 0.889 & -0.056 & 0.602 \\
& B & 0.732 & 0.629 & 0.103 & 0.335 \\
& C & 0.716 & 0.859 & -0.143 & 0.181 \\
\hline
\end{tabular}

Table 6.13: Differences in precision between the groups.

\begin{tabular}{lcc}
\hline System & Group 1 - Group 2 & p-value \\
\hline ANS & -0.112 & 0.201 \\
WinSpirs & -0.025 & 0.781 \\
\hline
\end{tabular}

\subsubsection{Precision}

The results of the ANOVA test for the variable precision (the fraction of the found documents that was relevant) are shown in Table 6.11. None of the sources of variance was significant, nor were the differences between ANS and WinSpirs for the various groups and questions (Table 6.12). The overall mean precision was 0.776 .

Table 6.13 shows that the systems do not show a difference in precision between the two groups. 
Table 6.14: Anova test for Rijsbergen's measure.

\begin{tabular}{lcrccc}
\hline Source of variance & SS & DF & MS & F & p \\
\hline Test person & 0.734 & 23 & 0.032 & 2.162 & 0.015 \\
System & 0.002 & 1 & 0.002 & 0.102 & 0.752 \\
Question & 0.041 & 2 & 0.020 & 1.383 & 0.262 \\
Question * system & 0.030 & 2 & 0.015 & 1.005 & 0.375 \\
Group * system & 0.056 & 1 & 0.056 & 3.779 & 0.059 \\
individual error & 0.620 & 42 & 0.015 & & \\
\hline
\end{tabular}

Legend: SS: sum of squares; DF: degrees of freedom; MS: mean sums of squares; $F: F$-ratio; p: significance of $F$.

Table 6.15: Rijsbergen's measure per group and per question.

\begin{tabular}{llllll}
\hline & & \multicolumn{2}{c}{ Rijsbergen's measure $(\alpha=0.5)$} & & \\
\cline { 3 - 4 } & & ANS & WinSpirs & Difference & p-value \\
\hline Group & & 0.776 & 0.856 & -0.080 & 0.057 \\
& 1 & 0.745 & 0.690 & 0.055 & 0.186 \\
Question & 2 & & & & \\
& $\mathrm{~A}$ & 0.804 & 0.773 & 0.031 & 0.538 \\
& $\mathrm{~B}$ & 0.726 & 0.828 & -0.102 & 0.048 \\
& $\mathrm{C}$ & 0.750 & 0.717 & 0.033 & 0.510 \\
\hline
\end{tabular}

Table 6.16: Differences in Rijsbergen's measure between the groups.

\begin{tabular}{lcc}
\hline System & Group 1 - Group 2 & p-value \\
\hline ANS & 0.031 & 0.441 \\
WinSpirs & 0.166 & 0.000 \\
\hline
\end{tabular}

\subsubsection{Rijsbergen's Measure}

In Subsection 6.2.2 a higher recall for ANS was found in group 1 and with question B. Because no significant differences in precision were found one may conclude that ANs' effectiveness was higher for the particular group and question. However, in this case we have compared average recall and average precision. A better approach compares recall and precision per case (i.e., the results of a question answered by one test person). Rijsbergen's measure makes this possible by combining recall and 
precision in one measure. It requires a constant $\alpha$ that indicates the importance of recall relative to precision. We arbitrarily chose $\alpha=0.5$, which means recall and precision are considered to be equally important.

Table 6.14 shows the ANOVA results for Rijsbergen's measure. The only significant source of variance was the factor test person. The significance of the interaction group * system is higher than for the variable recall, but still above the 0.050 level $(p=0.059)$.

In Table 6.15 the mean values for Rijsbergen's measure are shown for the two systems. Remember from Section 5.1.3 that a high value means a low effectiveness. The difference between the two systems for group 1, which was significant for the variable recall, is not significant for Rijsbergen's measure $(p=0.057)$. However, the advantage of ANS over WinSpirs for question B is still significant $(p=0.048)$.

Like recall, Rijsbergen's measure does not differ between the groups for ANS, but for WinSpirs it is worse for group 1 than for group 2 .

\subsubsection{Time Used}

Table 6.17 shows the ANOVA results for the variable time used. The interactions of question and group with system were significant $(p=0.002$ and $p=0.005$, respectively).

Table 6.18 shows that for group 1 there was no difference between the two systems. For group 2, the test persons were on average almost 4 minutes faster with WinSpirs than with ANS $(p=0.001)$. For the questions $A$ and $C$ the test persons were faster with WinSpirs than with ANs, almost 5 minutes $(p=0.001)$ and 4 minutes $(p=0.007)$, respectively. However, for question B the ANS users were almost 3 minutes faster $(p=0.039)$.

As can be seen in Table 6.19, no significant differences for the two systems were found between groups.

In $35 \%$ of the sessions the test person used the maximum allowed time. This raises the question whether parametric tests such as ANOVA and LSD can be used here. Therefore, also a nonparametric test, the stratified Mann-Whitney test, was used. We followed the procedure described by Senn (1993): for each test person, the difference between the average time with ANS and the average time with WinSpirs was determined. The differences of the test persons who used the systems in the sequence ANS, ANS, WinSpirs was tested against the differences of those who used the systems in the sequence WinSpirs, WinSpirs, ANs. Thus, sequence effects were eliminated. The other two sequence pairs were treated similarly. The resulting three test statistics were combined into one $\left(Z_{t}=0.250\right)$. The conclusion was that for time used there is no significant difference $(p=0.800)$ between ANS and WinSpirs. This confirms the outcome of the ANOVA in Table 6.17. Differences between groups and between questions cannot be determined with this method. 
Table 6.17: Anova test for time used.

\begin{tabular}{lrrccc}
\hline Source of variance & \multicolumn{1}{c}{ SS } & DF & MS & F & p \\
\hline Test person & 332.945 & 23 & 14.476 & 1.369 & 0.185 \\
System & 28.356 & 1 & 28.356 & 2.681 & 0.109 \\
Question & 51.985 & 2 & 25.993 & 2.458 & 0.098 \\
Question * system & 152.063 & 2 & 76.032 & 7.190 & 0.002 \\
Group * system & 94.900 & 1 & 94.900 & 8.974 & 0.005 \\
individual error & 444.136 & 42 & 10.575 & & \\
\hline
\end{tabular}

Legend: SS: sum of squares; DF: degrees of freedom; MS: mean sums of squares; F:F-ratio; p: significance of F.

Table 6.18: Time used per group and per question.

\begin{tabular}{lccccc}
\hline & & \multicolumn{2}{c}{ Time used (in min.) } & & \\
\cline { 3 - 4 } & & ANs & WinSpirs & Difference & p-value \\
\hline Group & 1 & 16.8 & 16.9 & -0.1 & 0.903 \\
& 2 & 18.6 & 14.8 & 3.8 & 0.001 \\
Question & & & & & \\
& $\mathrm{A}$ & 18.2 & 13.6 & 4.6 & 0.001 \\
& $\mathrm{~B}$ & 16.5 & 19.3 & -2.8 & 0.039 \\
& $\mathrm{C}$ & 18.4 & 14.6 & 3.8 & 0.007 \\
\hline
\end{tabular}

Table 6.19: Differences in time used between the groups.

\begin{tabular}{lcr}
\hline System & Group 1 - Group 2 & p-value \\
\hline ANS & -1.9 & 0.094 \\
WinSpirs & 2.1 & 0.059 \\
\hline
\end{tabular}




\subsubsection{User Satisfaction}

In Table 6.20 the ANOVA results for the variable user satisfaction are shown. The only significant source of variance is the factor question.

The test persons of group 1 were equally satisfied with ANS and WinSpirs. The test persons of group 2 were more satisfied with WinSpirs; the difference was almost 1 point on a scale of 5 . For question $\mathrm{A}$, the WinSpirs test persons were more satisfied than the ANS test persons; the difference was 1 point.

Table 6.22 shows that there were no significant differences between the two groups for the systems.

\subsubsection{An Extension of the Experimental Design}

During the experiment, a number of test persons ( 9 out of 24) mentioned the same missing feature in ANS: a Boolean AND-like means for combining key terms. It was therefore decided to incorporate such a feature in ANs (as described in Subsection 4.4.4), and to use this extended system with a new group of 12 second-year students (group 3). ${ }^{3}$ Because of the experimental design, the results of the group that used the extended ANS had to be analysed independent of the results of the other groups. The results reported in this chapter do not include group 3, unless explicitly stated. Unfortunately, there was no time to make the changes to the Information Network that were necessary to make optimal use of the new feature. ${ }^{4}$

The results for group 3 can be summarized as follows:

Recall: The recall of WinSpirs was 0.166 and the recall for ANs was 0.143 . The only significant difference was for question C: WinSpirs had a much higher recall than ANS (0.348 vs. $0.053 ; p=0.003)$. For one of the cases Cook's distance was greater than $0.5(0.587)$ : this test person had scored an exceptional high recall of 0.81 for question C..$^{5}$ After deletion of this case, the recall of WinSpirs for question $\mathrm{C}$ dropped to 0.254 which is still significantly better than ANS $(p=0.004)$. The overall recall of WinSpirs then dropped to 0.128 .

Precision: No significant differences between the systems were found.

Rijsbergen's measure: The only significant difference was for question C: WinSpirs had a much lower value than ANS $(0.582$ vs. $0.907 ; p=0.001)$. The case that had a high value of Cook's distance for recall did not have so for Rijsbergen's measure (0.273).

\footnotetext{
${ }^{3}$ It was not possible to find sufficient fourth-year students to fill a group of test persons; since it was the end of their term they had already left.

${ }^{4}$ The term for the second-year students was about to end. Postponing this second part of the experiment to the next term would render it difficult to compare the results with the first part.

${ }^{5}$ Surprisingly, this test person had no search experience in Medline whatsoever.
} 
Table 6.20: ANOvA test for user satisfaction.

\begin{tabular}{lrrrcc}
\hline Source of variance & SS & DF & MS & F & p \\
\hline Test person & 9.814 & 23 & 0.427 & 0.434 & 0.983 \\
System & 3.063 & 1 & 3.063 & 3.115 & 0.085 \\
Question & 7.750 & 2 & 3.875 & 3.941 & 0.027 \\
Question * system & 4.550 & 2 & 2.275 & 2.314 & 0.111 \\
Group * system & 2.007 & 1 & 2.007 & 2.041 & 0.160 \\
individual error & 41.297 & 42 & 0.983 & & \\
\hline
\end{tabular}

Legend: SS: sum of squares; DF: degrees of freedom; MS: mean sums of squares; $\mathrm{F}: F$-ratio; p: significance of $\mathrm{F}$.

Table 6.21: User satisfaction per group and per question.

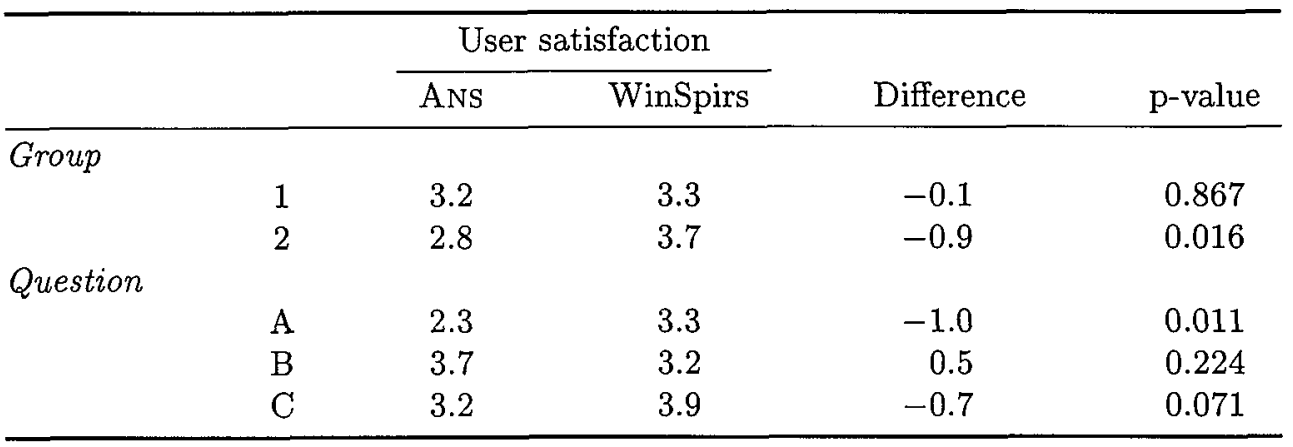

Table 6.22: Differences in user satisfaction between the groups.

\begin{tabular}{lcr}
\hline System & Group 1 - Group 2 & p-value \\
\hline ANS & 0.4 & 0.246 \\
WinSpirs & -0.4 & 0.246 \\
\hline
\end{tabular}


Time used: No significant differences between the systems were found. The interaction system * question was significant $(p=0.014)$, but no significant differences were found with the LSD test.

User satisfaction: No significant differences between the systems were found.

The relative high recall of WinSpirs for group 3 undermines the conclusion from Subsection 6.2.2 that the second-year students have difficulty with WinSpirs. The poor results of ANS for question $C$ suggest that the extension of ANS in fact appeared to be a deterioration.

\subsection{Analysis of Searches}

In the previous sections we concluded that the results for the three questions were different. Therefore, in this section we will examine the individual questions. The questions themselves are discussed in the appendix.

\subsubsection{Two Characteristic Cases}

Below we describe two cases from ANS' logs that are characteristic for the ANS searches as a whole. The first case shows a typical way of finding key terms with ANs. The second case illustrates a shortcoming in our use of UMLs.

\section{Finding Key Terms through Documents}

A pattern that can be observed regularly in ANS' logs is the identification of additional key terms through documents. As a case in point, we describe part of a session with question $A$.

The key term Smoke Adverse Effects was clicked on to show its neighbours, which were three documents. One document was marked relevant after its content was viewed. The test person proceeded with clicking on the document to see the remainder of its key terms in the browser. From these key terms Smoke inhalation injury was clicked and following one of its links, the test person found another relevant document.

This is a good example of finding a key term through browsing from key term to document to key term. It is also possible in query-oriented IR systems, but requires more work; in ANS it is a natural way of finding key terms.

\section{Disorientation Caused by UMLS}

Multiple test persons felt, at one time or another, as if they were walking in a labyrinth: they browsed through a number of very similar key terms, with many interconnecting links and without any documents linked to them. These parts of 
UMLS are somehow not fit for browsing with ANS since they disorient the user. The test persons encountered such parts with the questions $A$ and $B$, and especially with question $\mathrm{C}$.

We present an sample case with question C. Below an excerpt from the particular $\log$ is shown. Each action is preceded by a time tag (mm:ss) that indicates the time elapsed since the search started. The commands used are:

Select: Show the key term that was clicked in full if it was abbreviated.

Expand: Show the key terms related to the key term that was clicked.

Back: Go back to the previous situation.

08:43 Select Hypertension complicating pregnancy, childbirth, and the puerperium

08:46 Expand Hypertension complicating pregnancy, childbirth, and the puerperium

09:11 Expand Complications mainly related to pregnancy

09:41 Expand Infectious and parasitic conditions in the mother classifiable elsewhere, but complicating pregnancy, childbirth, or the puerperium

09:59 Expand Malaria complicating pregnancy, childbirth, or the puerperium

10:17 Back to Infectious and parasitic conditions in the mother classifiable elsewhere, but complicating pregnancy, childbirth, or the puerperium

10:19 Back to Complications mainly related to pregnancy

10:25 Back to Hypertension complicating pregnancy, childbirth, and the puerperium

10:40 Expand Eclampsia complicating pregnancy, childbirth or the puerperium, unspecified as to episode of care

10:44 Expand Eclampsia complicating pregnancy, childbirth or the puerperium, unspecified as to episode of care

10:58 Expand Hypertension complicating pregnancy, childbirth, and the puerperium

11:09 Back to Eclampsia complicating pregnancy, childbirth or the puerperium, unspecified as to episode of care

11:26 Select Hypertension complicating pregnancy, childbirth, and the puerperium 
11:36 Expand Hypertension complicating pregnancy, childbirth, and the puerperium

11:37 Expand Benign essential hypertension complicating pregnancy, childbirth, and the puerperium

12:14 Back to Hypertension complicating pregnancy, childbirth, and the puerperium

12:27 Expand Complications mainly related to pregnancy

13:19 Back to Hypertension complicating pregnancy, childbirth, and the puerperium

\section{3:48 Expand pregnancy toxemia/hypertension}

At time tag 08:43 the test person 'entered' the 'labyrinth': it consists of multiple key terms concerning hypertension and eclampsia. The key terms are long and homogeneous; it must have been difficult for the test person to decide in which way to continue browsing. After wandering 5 minutes, the test person found an 'exit'. Four minutes later, the test person entered the 'labyrinth' again, and spent 4 minutes until his 20 minutes search time were completed (the particular log excerpt is not shown here). Thus, the test person spent almost half of the searching time being lost in the 'labyrinth'!

\subsubsection{Differences in Retrieval Frequencies}

Two IR systems can have similar effectiveness and efficiency but can differ in the sort of documents they find. To find out if there are relevant documents that are easier to find with a particular system, for each document the frequency of finding it with the two systems was determined. The results are depicted in Figures 6.1, 6.2, and 6.3 for the questions A, B, and C, respectively. Each bar in the figures represents one document. Indeed, for several documents there is a considerable difference in finding frequency between the two systems. We compared for each relevant document by how many test persons it was found with ANS and by how many with WinSpirs. A high difference implies that one system is more suitable for finding the particular document than the other. For question A, the greatest difference was 6 (which is $50 \%$ of the maximum possible difference), both once in favour of ANS and once in favour of WinSpirs. For question B the maximum difference was $7(58 \%)$ in favour of ANs and $5(42 \%)$ in favour of WinSpirs. For question $\mathrm{C}$ the differences were smaller: the maximum difference was $2(17 \%)$ in favour of ANS and $4(33 \%)$ in favour of WinSpirs.

\subsubsection{Documents Found Only Once}

The figures in the previous section (Figures 6.1-3) suggest that many documents were found only once; that is, these documents were found by only one test person. 


\section{Question A}

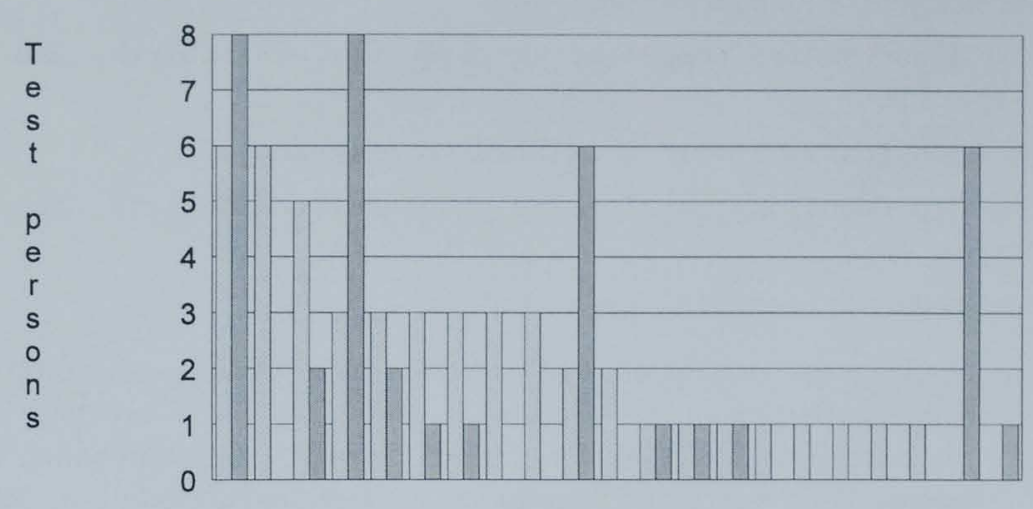

Documents

System

\section{WinSpirs}

ANS

Figure 6.1: The number of times each relevant document was found with a particular system (question A).

If a relevant document is found only once, it may be a chance hit. For instance, a test person composed a query in WinSpirs that returned several thousands of documents. Instead of reformulating the question, he marked a few documents from the list. One of these documents was judged relevant by the experts; it was not found by any of the other test persons because its key terms did not allow it to be discriminated from the irrelevant documents. In this case, we can speak of a chance hit. Documents that are chance hits are of less importance for the evaluation experiment because they were not actually found with the use of the search facilities of the IR system.

In Table 6.23 the precise numbers of documents are shown that were found only once. Column 'Group 3 excluded' shows the numbers for groups 1 and 2 together. For question $\mathrm{B}$ these numbers are large, but the differences between the systems is not. For question A the difference is considerable. Column 'Group 3 included' shows the corresponding numbers for groups 1, 2, and 3 together. The difference between ANS and WinSpirs in this case for the questions A and B is small, but for question $\mathrm{C}$ the difference is large. In total, with WinSpirs more documents were found only once than with ANS. It is tempting to exclude these documents from the effectiveness analysis, but this is not possible because we cannot be sure that they are really chance hits. 


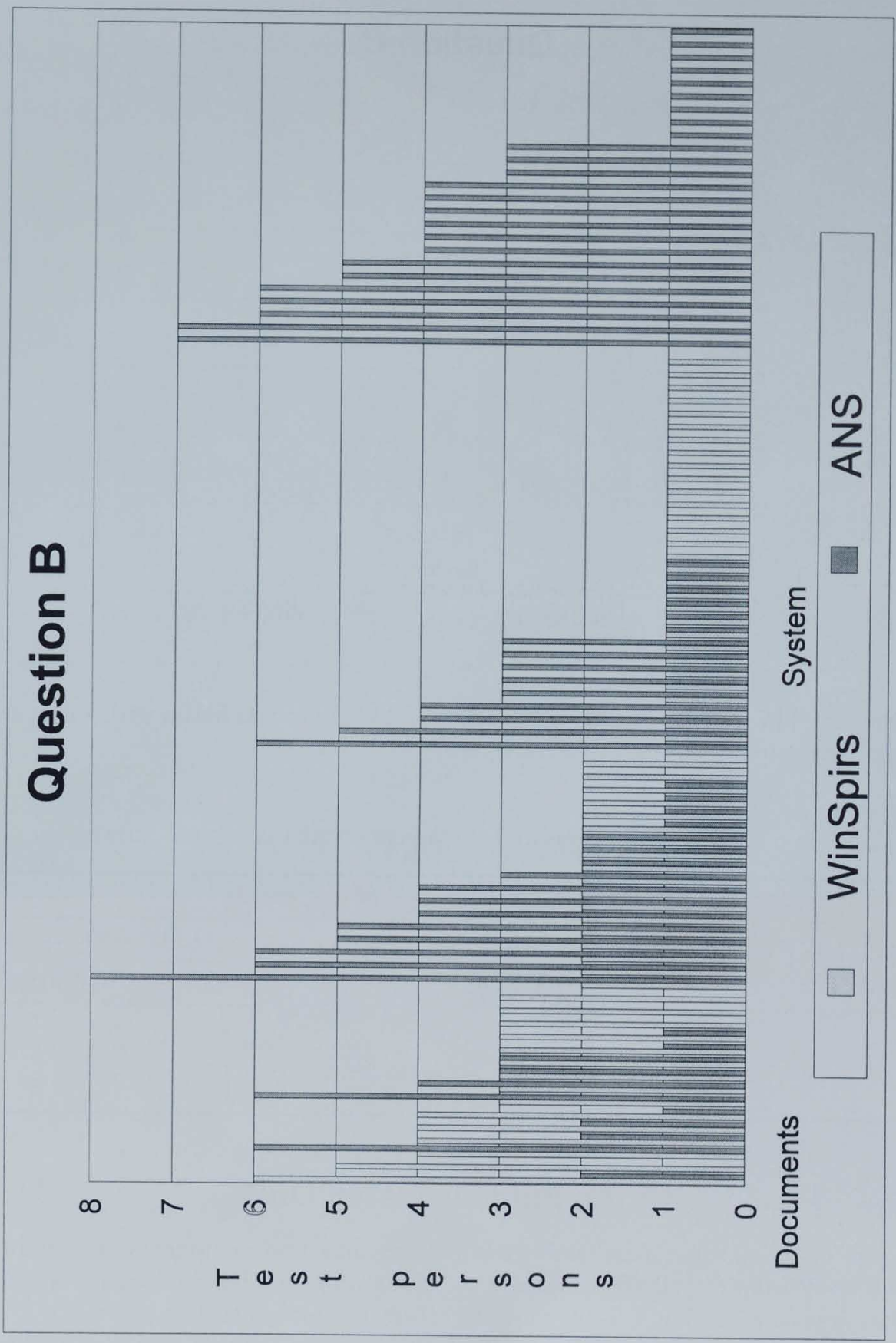

Figure 6.2: The number of times each relevant document was found with a particular system (question B). 


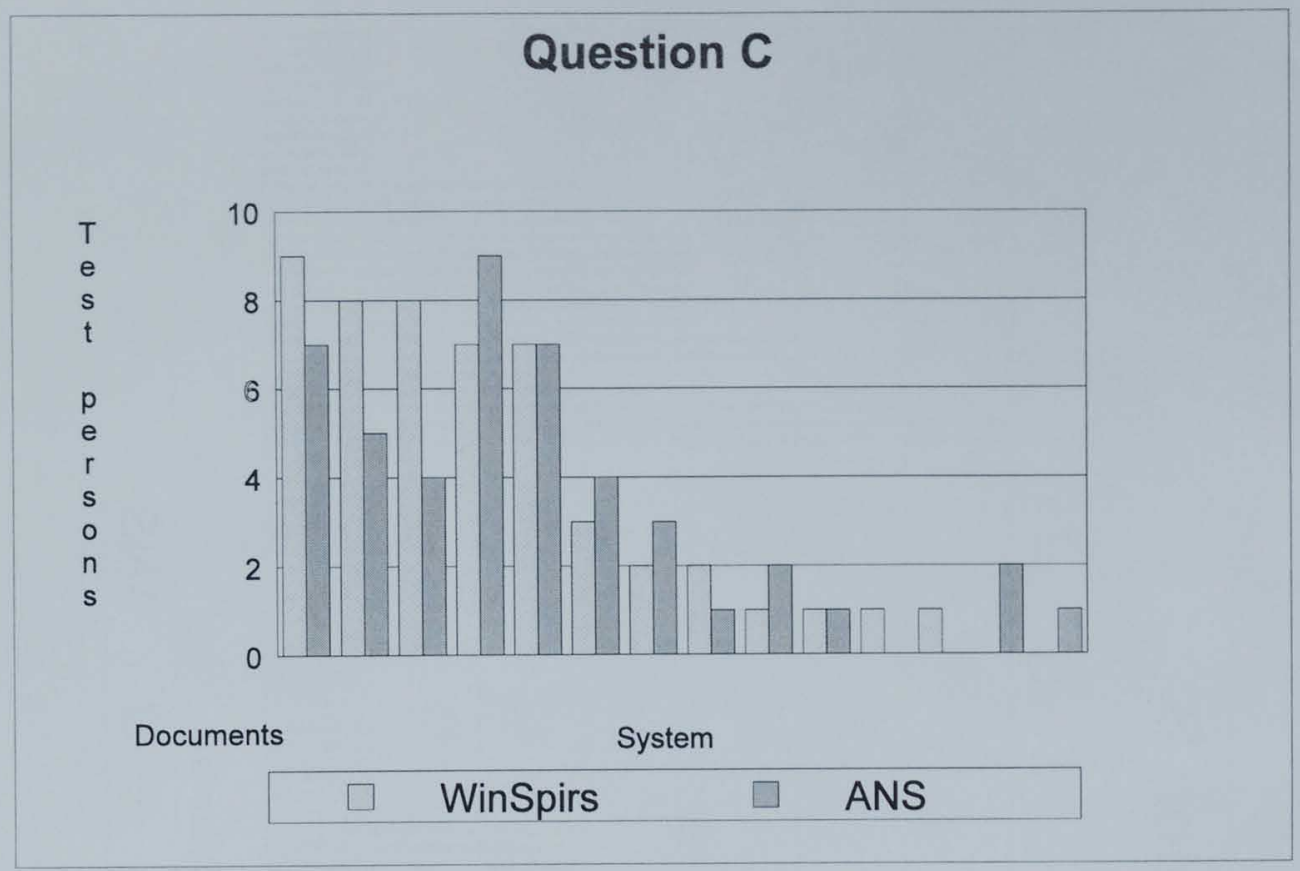

Figure 6.3: The number of times each relevant document was found with a particular system (question C).

Table 6.23: Number of relevant documents that were found only once.

\begin{tabular}{|c|c|c|c|c|}
\hline \multirow[b]{3}{*}{ Question } & \multicolumn{4}{|c|}{ Number of documents } \\
\hline & \multicolumn{2}{|c|}{ Group 3 excluded } & \multicolumn{2}{|c|}{ Group 3 included } \\
\hline & ANS & WinSpirs & ANS & WinSpirs \\
\hline A & 1 & 5 & 3 & 1 \\
\hline B & 7 & 10 & 3 & 6 \\
\hline $\mathrm{C}$ & 1 & 2 & 0 & 8 \\
\hline
\end{tabular}

\subsubsection{Found with the Extended ANS Only}

Despite of its shortcomings, the extended ANS may find documents that were not found by WinSpirs and the original ANS. That would indicate potential usefulness of the extension in that it leads users to otherwise unexplored documents.

To examine this we counted the number of documents that was found with the extended ANS only. For question A 2 additional documents were found, for question B 1, and 0 for question C. Each of these documents was found only once. 


\subsubsection{Correlations with User Satisfaction}

To examine whether the user satisfaction was not simply a reflection of the number of documents found, we determined the correlation between the user satisfaction and the number of documents found, using Spearman's rho. We also determined the correlation of user satisfaction with the variables that were investigated in Section 6.2. Table 6.24 shows that user satisfaction is correlated with recall, Rijsbergen's measure, the number of documents found (regardless of their relevance), and the time used. Group 3 was included in this analysis. There is no significant correlation with precision. All significant correlations are weak, the strongest correlation being time used $(-0.449)$.

The results are roughly as could be expected: test persons cannot estimate the recall they scored, since they do not know the number of relevant documents for a question. However, if a search goes well, both recall and user satisfaction are likely to be (but not necessarily) high. The same reasoning holds for Rijsbergen's measure. Since a test person is responsible for marking documents as relevant, a test person scores, in his own view, a precision of 1.00. This explains the absence of a correlation between precision and user satisfaction. It was to be expected that the more documents a test person marked as relevant, the higher his satisfaction would be. Nevertheless, the correlation is rather weak. Therefore we conclude that user satisfaction is not solely dependent on the number of documents found. The time used had also a significant correlation; obviously, a smoothly executed search leads to a higher user satisfaction than one that was executed ponderously.

Table 6.24: Correlations with user satisfaction (Group 3 included).

\begin{tabular}{lcr}
\hline & \multicolumn{2}{c}{ Spearman's rho } \\
\cline { 2 - 3 } Variable & Correlation Coefficient & p-value \\
\hline Recall & 0.362 & 0.000 \\
Precision & -0.055 & 0.569 \\
Rijsbergen's measure & -0.362 & 0.000 \\
Found & 0.374 & 0.000 \\
Time used & -0.449 & 0.000 \\
\hline
\end{tabular}

\subsection{Search Behaviour in ANS}

With each of the 3 groups of 12 test persons a total of 18 ANS searches was conducted. Table 6.26 shows how often the test persons used various commands. Because no real differences were found between the groups or between the questions we do not show separate tables for groups or questions. The commands shown in the table are: 
Find by name: The number of times a selection of the list of available key terms was made. Every session starts with this command, but the first time is not counted.

Expand: The number of times a key term was clicked to show its related key terms.

Back: The number of times the backtrack facility was used to backtrack.

Forward: The number of times the backtrack facility was used to undo a backtracking action.

Show content: The number of times the content of a document was presented.

Show description: The number of times the description of a concept was presented.

Select filter pair: The number of times a different filter pair was chosen.

View combination: The number of times the result of the combination of key terms was viewed. This action was available to group 3 only.

The columns show the mean, median, standard deviation, range, and corrected mean. The latter is the mean of frequency corrected for the time used. Since the time used per test person varied, for some actions the mean may not give a proper picture of the actual usage. We define the corrected frequency as:

$$
f_{c o r}=f \frac{t_{\max }}{t}
$$

where $f$ is the frequency of the action, $t$ is the time used by the test person for the particular session, and $t_{\max }$ is the maximum time allowed. The value of $t_{\max }$ is always 20 (minutes).

Table 6.25: User actions in ANs.

\begin{tabular}{lrrrrr}
\hline Action & Mean & Median & Std. dev. & Range & Mean corr. \\
\hline Find by name & 2.59 & 2 & 2.92 & $0-13$ & 2.91 \\
Expand & 33.80 & 32 & 14.29 & $11-70$ & 38.97 \\
Back & 7.69 & 6 & 6.17 & $0-23$ & 8.68 \\
Forward & 0.04 & 0 & 0.20 & $0-1$ & 0.04 \\
Show content & 9.37 & 4 & 13.75 & $0-67$ & 11.51 \\
Show description & 1.71 & 1 & 3.05 & $0-15$ & 1.91 \\
Select filter pair & 3.37 & 3 & 2.33 & $1-10$ & 3.97 \\
View combination & 2.83 & 2 & 3.09 & $0-12$ & 3.10 \\
\hline
\end{tabular}


Find by name was not often used. This is a good sign; a high number would indicate that the browsing actions did not lead to satisfying key terms. However, in $31 \%$ of the cases the command was not used at all (the command was executed before the start of each session). This might well mean that at least some test persons did not realise that the command existed. The expand command was, of course, often used. The back command was also well used: one back command was issued for every five expand commands. Surprisingly, the forward command was hardly used. It is situated in the user menu next to the back command; thus, if a test person can find the back command, he must be able to find the forward command. Apparently, there was no need for this command. The mean of the use of the show content command is considerably higher than the median; some test persons made extensive use of the command, other test persons did not use it at all. The low median suggests that the key terms in the Information Network often give sufficient information about the content of the documents. The show description command is not often used. In half of the sessions it is not used at all, in most of the other sessions it is used only once. A possible explanation for this shortcoming is the limited number of available explanations; in two-thirds of the cases that the command was issued, no description was available. Half of these concerned key terms of the mainsub type; the associated main headings did have a description. Another shortcoming was the quality of the descriptions; they can be very abstract and therefore not elucidating to a student. A good example is the explanation of smoke: "A colloid system in which one or more solids is dispersed in a gas or vapor (Dorland's Illustrated Medical Dictionary 27th edition)." In all sessions the select filter pair command was used at least once. The view combination command was only available to group 3 . All test persons used it, but not very often.

\subsection{Search Behaviour in WinSpirs}

For each of the 3 groups of 12 test persons 18 WinSpirs searches were conducted. Table 6.26 shows how often the test persons used various WinSpirs features. Because no real differences were found between the groups or between the questions we do not show separate tables for groups or questions. The meanings of the rows are:

Queries: The number of queries made. ${ }^{6}$

AND: The number of times the AND operator was used. If AND occurred $n$ times in a query this counted as $n$ times.

OR: The number of times the OR operator was used. If oR occurred $n$ times in a query this counted as $n$ times.

\footnotetext{
${ }^{6}$ WinSpirs processes a query of the form $a b$ that is entered without the help of the thesaurus by first processing the queries a and $b$. The result is that this query counts as three queries. Consequently, the number of queries shown here should be regarded as an upper limit of the actual number.
} 
Explode: The number of times a key term was 'exploded'. When a key term is 'exploded', WinSpirs not only searches for the key term, but also for documents that contain any of its narrower key terms that are present in the thesaurus.

Full-text: The number of full-text queries made. Full-text query means here: the query is matched not only against the key terms assigned to a document, but also against the other fields, including title and abstract. Key terms that are entered without using the thesaurus and without explicitly stating which fields are to be searched constitute by default a full-text query. A query constructed by selecting a key term from the thesaurus is not a full-text query but a field query. Unfortunately, the limited user logs of WinSpirs do not allow us to distinguish between them.

All subheadings: The number of times a key term was used with all of its subheadings (the concept of subheadings was explained in Subsection 4.2.1).

Specific subheadings: The number of times a key term was used with none or some (but not all) of its subheadings.

The OR operator is not often used. Apparently, the test persons preferred to read the results of two separate queries over reading the disjunction of the results. The latter was of course not possible where the test person based the second query on the results of the first. Explosions of key terms were often used. This may be because WinSpirs automatically explodes a key term when the thesaurus is showing the narrower terms of the key term. A considerable number of full-text queries was performed compared to field queries. Often, test persons started with full-text queries and later turned to field queries. Specific subheadings are used as often as all subheadings. A good strategy to apply is first to use all subheadings, and then, when too many documents are found, to select specific subheadings.

The possibility of combining key terms in ANs limits the user to use only one AND (as was discussed in Subsection 4.4.4). We expected that this limitation would

Table 6.26: User actions in WinSpirs.

\begin{tabular}{lrccc}
\hline Action & Mean & Median & Std. dev. & Range \\
\hline Queries & 10.85 & 10 & 6.15 & $2-31$ \\
And & 3.35 & 3 & 3.04 & $0-16$ \\
Or & 0.91 & 0 & 2.28 & $0-13$ \\
Explode & 2.24 & 2 & 1.78 & $0-8$ \\
Full-text & 2.96 & 2 & 3.63 & $0-18$ \\
All subheadings & 1.83 & 1 & 1.78 & $0-7$ \\
Specific subheadings & 1.91 & 2 & 1.70 & $0-7$ \\
\hline
\end{tabular}


Table 6.27: Usage of single and multiple AND connectives in WinSpirs.

\begin{tabular}{lcc}
\hline Group & Single AND & Multiple ANDS \\
\hline 1 & 58 & 4 \\
2 & 49 & 7 \\
3 & 43 & 7 \\
\hline Total & 150 & 18 \\
\hline
\end{tabular}

not be a real disadvantage compared to a Boolean IR system. Although the latter system allows multiple AND connectives to be used in one query, we assumed that users would do this infrequently.

The latter assumption was tested by inspecting the logs of the WinSpirs searches that were conducted by the test persons. For each group of 12 test persons 18 WinSpirs searches were conducted. Table 6.27 shows for each of the three groups how many times single and multiple ANDs were used. A query that contained only one AND but referred to a query that also contained an AND was counted as multiple AND (e.g., \#5 AND \#6, where \#5 refers to Eclampsia AND Mother).

From the total of 17 occasions where multiple ANDs were used, 11 times one or more documents were found by the system. Our logs do not allow us to see how many of these documents were actually marked as relevant by the test persons. Since at no more than 11 occasions multiple ANDs were used during the WinSpirs searches, we conclude that allowing a single AND in ANS is sufficient.

\subsection{Interviews}

Each test person was asked several questions concerning his session. We only describe the most important results.

\subsubsection{Information Retrieval Experience}

The protocol excludes students from the evaluation who had much experience with ANS or (Win)Spirs (Subsection 5.2.4). Table 6.28 shows the search experience of the test persons per group. The following levels of experience were distinguished: no experience; ${ }^{7}$ the test person had followed the Medline introductory course; the test person did not follow the course, but used Spirs a few times; the test person followed the course and used Spirs a few more times; the test person followed the course and

\footnotetext{
${ }^{7}$ The students of group 2 mentioned that the course had not been very useful. Groups 1 and 3 were more satisfied.
} 
Table 6.28: How experienced are the test persons with Medline?

\begin{tabular}{llcl}
\hline & \multicolumn{3}{c}{ Group } \\
\cline { 2 - 4 } Experience & 1 & 2 & 3 \\
\hline No & 2 & 0 & 2 \\
Course & 8 & 1 & 5 \\
Few times & 0 & 2 & 1 \\
Course + few times & 2 & 6 & 4 \\
Course + several times & 0 & 3 & 0 \\
\hline
\end{tabular}

used Spirs several times. In the Medline introductory course the system used was Spirs, the MS DOS version of WinSpirs.

The members of groups 1 and 3 had, as could be expected, about the same experience. The members of group 2 were more experienced. At the time of the evaluation, these students were doing a scientific internship for which many of them had to access medical literature.

\subsubsection{Missing Features and Other Complaints}

The test persons were asked if they had missed any features in ANS or if they had other suggestions for improvement.

Firstly, a combination facility to combine key terms in an AND-like way was desired. This was mentioned by two test persons from group 1 and seven test persons from group 2. A possible explanation for the difference between second and fourth year is that the fourth-year students had more experience with Boolean search than the second-year students. The extended ANS was built to meet this wish. Unfortunately, only second-year students were available for the extended ANS evaluation, whereas particularly the fourth-year students had asked for it.

Secondly, test persons complained that the display was cluttered: one test person from group 1, three from group 2, and eight from group 3. An explanation for the high number for group 3 might be that in the extended ANS most of the other complaints had been dealt with; the cluttered display may have been the 'only' problem left to complain about. Although the combination facility did not improve searching, the test persons did not complain about it. The most important reasons for the cluttered-display complaint were:

- The neighbours of a node are displayed more or less in a circle around the node. It was felt that a list would be more convenient for nodes with many neighbours.

- Often there was too much information on the screen. 
- It should be possible to remove the parts of the Information Network that have been explored and are of no interest (anymore).

Incidental remarks were:

- Instead of showing node labels in the bookmark list, titles must be shown. This was realised in the extended ANS.

- The display should show how many neighbours a particular node has. This feature was added in the extended ANs.

- It should be clear from which concepts the documents were inspected. This was partly realised in the extended ANS: nodes that have never been clicked on in the current session have a different type face than nodes that have been clicked at least once. Thus, it is clear whether the documents of a node have been inspected.

- The combination facility lacks an equivalent of the 'explosion' of a concept in WinSpirs, which includes in the query all children of the concept.

\subsubsection{Link Type Usage}

An important feature of ANS is its ability to show the type of relation between two nodes. The test persons were asked whether they had looked at the types during browsing, and whether this had been useful. Table 6.29 shows that most test persons had looked at the link types and found them useful.

Table 6.29: How often did the test persons use the link types and how often was it useful?

\begin{tabular}{lrcccccc}
\hline & \multicolumn{3}{c}{ Used } & & \multicolumn{3}{c}{ Useful } \\
\cline { 2 - 4 } Group & Yes & Sometimes & No & & Yes & Sometimes & No \\
\hline 1 & 12 & 0 & 0 & & 8 & 2 & 2 \\
2 & 8 & 3 & 1 & 6 & 3 & 2 \\
3 & 10 & 0 & 2 & 6 & 3 & 1 \\
\hline
\end{tabular}

\subsubsection{Filters}

One of the conclusions of the informal evaluation (Section 3.5) was that the filter mechanism was not clear to the test persons and, therefore, was not used. Filter pairs, a simple version of the filter mechanism, were incorporated in the current evaluation. Test persons were asked if the filter pairs were clear. Table 6.30 shows that the majority of the test persons experienced no problems with the new mechanism. 
Table 6.30: Did the test persons find the filter pairs mechanism clear?

\begin{tabular}{lrcc}
\hline & \multicolumn{3}{c}{ Filters clear } \\
\cline { 2 - 4 } Group & Yes & Somewhat & No \\
\hline 1 & 8 & 3 & 1 \\
2 & 10 & 2 & 0 \\
3 & 11 & 0 & 1 \\
\hline
\end{tabular}

\subsubsection{Usage of the WinSpirs Thesaurus}

It was not possible to log the usage of the WinSpirs thesaurus. The logs only show the queries that the test persons formulated. Therefore the test persons were asked whether they had used the thesaurus and if so, how useful it was. All test persons answered they had used it and found it useful. Although the logs did not show how the thesaurus was used, the form of the query indicates when the thesaurus was used. Queries that are constructed using the thesaurus always have an indication of subheadings. Naturally, the test person may have typed this himself, but this is not very likely, especially considering the limited experience of the test persons. Inspection of the logs suggests that the thesaurus was used often, but not by all test persons for all search questions.

\subsubsection{Difficulty with English in the Search Questions}

The search questions were formulated in Dutch whereas ANS and WinSpirs use English. We asked the test persons whether they had experienced any difficulty with finding the English terms corresponding with the Dutch search questions. Table 6.31 shows that almost half of them had no problems. The 12 test persons who had, reported 'fracture' as the most important problem. These were second-year as well as fourth-year students. ${ }^{8}$

Table 6.31: Did the test persons experience difficulties with the English terms?

\begin{tabular}{lccc}
\hline & \multicolumn{3}{c}{ Difficulties } \\
\cline { 2 - 4 } Group & Yes & Somewhat & No \\
\hline 1 & 1 & 4 & 7 \\
2 & 0 & 7 & 5 \\
3 & 1 & 6 & 5 \\
\hline
\end{tabular}

\footnotetext{
${ }^{8}$ In the search question the popular term 'botbreuk' was used. The medical term is 'fractuur', very similar to the English 'fracture'.
} 


\subsubsection{Clarity of the Search Questions}

To obtain reliable test results requires from the test persons to have the same perception of the search questions. Therefore it is important that the test persons have understood the search questions well. We asked the test persons how clear they found the questions. Table 6.32 shows how many test persons found the questions clear, not entirely clear, and unclear. An ample majority found the questions clear. There were some problems with question $A$, where the word 'smoke' caused some confusion, and with question $\mathrm{C}$, where it was not clear how wide 'eclampsia-related' had to be interpreted.

Table 6.32: Were the search questions clear to the test persons?

\begin{tabular}{|c|c|c|c|c|c|c|c|c|c|}
\hline \multirow[b]{3}{*}{ Group } & \multicolumn{9}{|c|}{ Question clear } \\
\hline & \multicolumn{3}{|c|}{$\mathrm{A}$} & \multicolumn{3}{|c|}{$\mathrm{B}$} & \multicolumn{3}{|c|}{$\mathrm{C}$} \\
\hline & Yes & Not really & No & Yes & Not really & $\mathrm{No}$ & Yes & Not really & No \\
\hline 1 & 8 & 1 & 3 & 11 & 1 & 0 & 12 & 0 & 0 \\
\hline 2 & 9 & 2 & 1 & 10 & 1 & 1 & 10 & 1 & 1 \\
\hline 3 & 9 & 3 & 0 & 10 & 2 & 0 & 7 & 5 & 0 \\
\hline
\end{tabular}

\subsubsection{Learning Effect}

The instruction and practice time for both systems was short. We expected this to be a more severe constraint for ANS than for WinSpirs because ANS' interface is so different from the programs that students are used to work with, and because most students had followed the Medline course. To assess this, we asked those test persons who answered two search questions with ANS whether they thought that the second question with ANS went smoother than the first because they were more accustomed

Table 6.33: Were the test persons more accustomed to the IR system that they used for the second time?

More accustomed

\begin{tabular}{lccccccc}
\cline { 2 - 4 } & \multicolumn{3}{c}{ ANs } & & & \multicolumn{3}{c}{ WinSpirs } \\
\cline { 2 - 4 } \cline { 6 - 7 } Group & Yes & Somewhat & No & & Yes & Somewhat & No \\
\hline 1 & 3 & 1 & 2 & & 1 & 5 \\
2 & 3 & 1 & 2 & 2 & 0 & 4 \\
3 & 5 & 1 & 0 & & 2 & 1 & 3 \\
\hline
\end{tabular}


to system. A similar question was asked to test persons who answered two questions with WinSpirs. Table 6.33 confirms that more double-ANs test persons experienced an improvement of their skills with the program than double-WinSpirs users did (14 out of 18 vs. 6 out of 18).

\subsection{Conclusions on the Experiment}

In Subsection 5.2.3 we formulated four hypotheses. With the results of Section 6.2 we can now verify them:

1. ANS is more effective than WinSpirs.

Conclusion: In the overall comparison, ANS and WinSpirs are equally effective. Hence, the hypothesis must be rejected. However, for one individual question ANS is more effective, and also for the second-year students one may argue that ANS is more effective.

2. ANS is less efficient than WinSpirs.

Conclusion: ANS is less efficient than WinSpirs for fourth-year students, and ANS is more efficient than WinSpirs for second-year students. Hence, the hypothesis must be rejected.

3. The test persons are more satisfied with ANS than with WinSpirs.

Conclusion: In the overall comparison, the test persons are equally satisfied with ANS and WinSpirs. However, the fourth-year students are more satisfied with WinSpirs than with ANS. Hence, the hypothesis must be rejected.

4. The advantage of ANS over WinSpirs in terms of effectiveness, efficiency, and user satisfaction is greater for second-year students than for fourth-year students.

The hypothesis cannot be rejected.

Other conclusions that follow from the results are:

- The results for recall (Subsection 6.2.2), precision (Subsection 6.2.3), and Rijsbergen's measure (Subsection 6.2.4) suggest that beginning students benefit more from the domain knowledge in ANS than advanced students do. Another explanation for ANS' better results for beginning students may be that advanced students are more experienced with (Win)Spirs. However, Subsection 6.6.1 indicates that the differences in experience are small.

- (Subsection 6.2.2) The overall mean recall was quite low: 0.152 . This is even more disappointing considering that this is not the absolute recall; there are probably relevant documents in the document set that were never found. Therefore the actual recall is even lower. 
- (Subsection 6.2.6) It is striking that in general the test persons are quite satisfied with their results, although the recall they scored is poor.

- (Subsection 6.2.7) The extension of ANS was not an improvement. It is not clear whether this was due to the combination facility itself or to the insuitability of the Information Network for the combination facility.

- (Subsection 6.3.1) The possibility of browsing from key term to document to key term is an asset. Some parts of UMLS hamper browsing instead of facilitating it.

- (Subsection 6.3.2) Relevant documents found more often with one system than with the other exist. However, we cannot say that, concerning this aspect, one system is really different from the other.

- (Subsection 6.3.3) For groups 1 and 2, the recall of question A may be somewhat flattering because several documents may have been found with WinSpirs by chance. For group 3, WinSpirs had a considerable number of these possible chance hits with question $\mathrm{C}$. This observation puts in perspective the difference in recall between ANS and WinSpirs for question C. A possible explanation for the high number of possible chance hits for WinSpirs is that it is relatively easy for the WinSpirs user to read a few random documents from a large query result. Another source may be WinSpirs' full-text abstract search facility.

- (Subsection 6.3.4) Documents uniquely found with the extended ANS were all found only once. This indicates that the extended ANs does not lead the user to documents that cannot be found otherwise.

- (Subsection 6.3.5) The user satisfaction has a weak correlation with several of the other variables examined. However, it does not solely depend on one of them.

The conclusions above will be further discussed in the next chapter. 



\section{Chapter 7}

\section{Discussion and Conclusions}

In this final chapter we summarize and discuss the results and conclusions of the thesis. We follow the hypotheses and research questions that were formulated in Section 1.5. In Section 7.1 the first group of research questions is answered and in Section 7.2 the second group. The results of the hypotheses and the problem statement are discussed in Section 7.3. Finally, Section 7.4 provides directions for future research.

\subsection{The Information Network and the Browser}

In the subsections that follow, the eight research questions regarding the realisation of the Information Network and the graphical meta-information browser are discussed.

\subsubsection{Documents}

What is, in the context of the ARCHIMEDES project, a document? How and when are documents distinguished from one another? What types of documents exist?

Before we could design a representation for document content and location knowledge, it was first necessary to know which meanings of 'document' had to be supported by ANS. This subject was explored in Section 3.1. The results are possibly of use to other information systems as well. A document is defined as stored information that is declared to be a document by its producer. A special kind of document is defined, called formula, for expressions in formal languages. Two particular kinds of formula documents are databases and knowledge bases. Moreover, a distinction is made between static documents and documents that are subject to change, such as databases. 


\subsubsection{IR Systems}

What are characteristics of existing IR systems? Which of the characteristics are of interest for ARCHIMEDES?

These questions are answered in Chapter 2. It provides a set of characteristics for the comparison of query-oriented IR systems known from the literature. We used this set to decide which characteristics ANS should possess. The most important characteristic is the IR model. The IR models discussed were not suitable for ANS; our idea of browsing meta-information does not fit into them. Moreover, these models are not suitable for browsing. Therefore, we defined a new model: the metabrowse model. The systems described by Collier (1987) and Hemmje et al. (1994) can be considered to use this model. However, the expressiveness of their InformationNetwork equivalents is less than the ANS Information Network. Furthermore, they lack a filter mechanism.

\subsubsection{Architecture of ANS}

What should the architecture of the ARCHIMEDES system be?

Chapter 3 is about the design of ANS. The design of ANs' architecture is presented in Section 3.2. It is based on an extension of the relational database that provides for meta-information about the documents. The database is accessed through a browsing and a querying mechanism. Document contents are presented by external programs managed by ANS. The actual implementation is described in Chapter 4. Only a rudimentary query tool was realised; research concentrated on the browser tool.

\subsubsection{The Information Network}

How should the Information Network be defined? How should it be specified?

Section 3.3 defines the Information Network in terms of a relational database. It consists of an RDBMS with (1) a metaschema that is fixed for all Information Networks, (2) a conceptual schema that is constrained by the metaschema and that depends on the domain and application of the Information Network, and (3) a fact base that is constrained by the conceptual schema and that contains the metainformation proper. In the actual implementation (described in Section 4.1) the metaschema is not explicitly represented. It is implicitly assumed (by means of a hard-wired syntax checker), and differs on a number of points from the original which is specified in Figure 3.3; it is more constrained. The conceptual schema is merely implicitly represented; it is the responsibility of the administrator to make the fact base consistent. In a final version of ANS, these parts are to be realised explicitly. 


\subsubsection{The Access Problem}

How can uniform access be realised?

In Subsection 3.2.1 the access problem is divided into two parts: retrieval and presentation. For retrieval, a standard addressing scheme is used (URLs). For presentation, standard document access programs (discussed in Subsection 3.1.1) are used; thus new document formats can be added at will. The disadvantage of this approach is that the user is confronted with multiple programs, each with its own user interface.

\subsubsection{Hypermedia in ANS}

How do the concepts of hypertext and hypermedia fit into ARCHIMEDES?

Hypertext and hypermedia are increasingly important ways to structure information. A general IR system as ANS should at least support them. In Chapter 1 we assumed that they would blend in with the ARCHIMEDES approach. This was confirmed in Subsection 4.2.2, which describes an Information Network that contains hypermedia from the Web.

\subsubsection{Browsing the Information Network}

How can the Information Network be visualized and browsed?

The Information Network is a large graph. A great deal of research has been carried out concerning visualization of graphs. One particular algorithm was selected and adapted to the demands of the application. The graph is too large to be displayed fully. There are three possible solutions to this problem: (1) enable the user to scroll through the graph, (2) enable the user to zoom out for an overview and zoom in for details, and (3) only show the direct neighbourhood of the node that the user last visited. After experimenting with these solutions the third one was adopted. Further experimentation concerned the initial positioning of nodes, the definition of 'the direct neighbourhood of the node that the user last visited' and the number of iterations that the algorithm should make. The resulting algorithm produces acceptable graphs. Possible improvements are discussed in Section 7.4.

Subsection 4.4.2 describes how the Information Network is visualized. Subsection 4.4.1 describes a typical browsing session.

\subsubsection{Navigation}

How can disorientation of the user be prevented while browsing through the Information Network?

Browsing through hypertext is often associated with disorientation. Also for the ANS browser this issue is imminent. Solutions used in hypertext systems were applied in ANS, as described in Subsection 4.4.3. More navigation aids than currently 
available in ANS could be incorporated (e.g., landmarks and fish-eye view). However, adding more 'gadgets' will make the operation of ANS more difficult for the user. On the basis of the system logs (Section 6.4) and the interviews of the test persons (Section 6.6) it can be concluded that the navigation aids were clear and well-used.

\subsection{The Evaluation}

In the following, the research questions regarding the performance of ANS are discussed.

\subsubsection{IR System Evaluation}

How does one evaluate an IR system?

Section 5.1 gives an overview of the IR system evaluation literature. An evaluation experiment where all variables are controlled is called a laboratory experiment. An uncontrolled experiment, with real users, real questions, and real documents is an operational experiment. In the former experiment observed results can be better linked to particular causes; the latter is more realistic.

The usual measures for IR system effectiveness are recall and precision. Recall is difficult to determine because the relevant documents, a subset of the document set, must be known. Another problem is that effectiveness has shown to be document-set dependent; therefore, system effectiveness is difficult to compare across experiments. Both problems can be alleviated if a second system is included in the evaluation experiment. Because recall and precision are dependent measures, and one of them is not informative without the other, various combined measures have been proposed. One of them is Rijsbergen's measure. Next to system effectiveness, also efficiency and user satisfaction can be determined.

The central notion in IR evaluation is relevance. The method of determining the relevance of a document depends on how one defines relevance. Various definitions exist; none is completely satisfactory. A commonly applied method involves independent judges assessing the documents' relevance to a question.

\subsubsection{Procedure for ANS Evaluation}

How to evaluate the ARCHIMEDES system?

We evaluated ANS by comparing it with WinSpirs, a commercially available IR system. We chose WinSpirs because it is used in our library. The document set was a selection of 36,000 documents from the biomedical literature database Medline. Test persons were divided into 2 groups of 12 persons: group 1 consisted of secondyear medical students, group 2 of fourth-year medical students. Domain experts formulated 3 questions. Each test person had to answer one question with ANS and two with WinSpirs or vice versa. Two domain experts per question judged the 
relevance of the retrieved documents. Using these judgements, we could determine the effectiveness, expressed in recall, precision, and Rijsbergen's measure. Efficiency was determined by measuring the test persons' search time (with a maximum of 20 minutes). User satisfaction was determined by asking the test persons to give a score on a Likert scale for each search question. During the experiment a third group, consisting of second-year medical students was added. This group used an extended version of ANS, which included an AND/OR-like combination facility.

Now that the evaluation has been completed and the results have been analysed, we can evaluate the evaluation. First we mention three shortcomings that we identified:

- Subsection 6.6.7 shows that most test persons found the questions clear. We assume that the clearness would have been even better if the questions would have had more context. Belkin (1981) calls this 'the desire of the user'. It was considered (see the appendix) to use questions asked in educational groups. However, these questions were not suitable because they were not sufficiently precise and it would be difficult to select questions for which sufficiently relevant documents were present in the Medline selection. Embedding the questions in (hypothetical) clinical cases was also a possibility. However, this might make the questions more complicated and therefore the results too dependent on the users' knowledge and intelligence.

- Given the fact that for $30 \%$ of the searches the maximum time of 20 minutes was used, a higher maximum might have been better.

- The results of group 3 with the extended ANS would have been better if the system would have been tested better. However, there was no time to do this since the academic year was about to end for the students.

We evaluated ANS using the Medline Information Network. How suitable was this Information Network? Firstly, we consider the document set, which consists of the January-February 1995 records of Medline. From an evaluation point of view, the size of the document set was reasonable; many experiments have been carried out with less documents (see Subsection 5.1.4). From the users' point of view, the size of the document set was manageable. The strength of ANS might have been more evident if the Medline documents had exploited links between documents; hence, it would have been possible to find directly documents related to a particular document.

Secondly, we consider the domain representation, which was constructed from the UMLS thesaurus. The coverage was sufficient. This is not surprising, since Medline documents are indexed with MesH, a subset of umLs. It is also MesH that constitutes the WinSpirs thesaurus. We had expected that the extra concepts from UMLS, although not used for indexing, would help the user to get to the desired MesH concepts. This has proven to be true only partly; the disadvantage is that 
the extra terms face the user with extra choices to be made concerning the browsing path. What is actually needed is a carefully made selection of extra concepts instead of the entire UMLS. Whereas UMLS has too many concepts, it has not a sufficient number of links. For instance, there are no links between diseases and their treatments. In Section 7.4 we propose a possible solution to generate some of these links automatically.

The relevances of the documents were judged by experts. How good were the judgements? The agreement between the experts is an indication of the quality of the judgements. In Section 6.1 we concluded that the agreement was high.

ANs was evaluated by comparing it to WinSpirs. But how good is WinSpirs? Greschner et al. (1996) compared WinSpirs ${ }^{1}$ with Knowledge Finder, another Medline IR system. The most important characteristic distinguishing Knowledge Finder from WinSpirs is its natural-language interface. Test persons (physicians and students) had to perform their search first with WinSpirs and then with Knowledge Finder, or vice versa. The relevance of the retrieved documents was ranked by each test person self. The users preferred the results of Knowledge Finder more often than those of WinSpirs; therefore it is concluded that Knowledge Finder has a higher effectiveness than WinSpirs. However, note that the relevance established by Greschner differs from the relevance established in the ANS evaluation (see also Section 5.1.7).

A larger evaluation was conducted by Haynes et al. (1994). In the experiment 27 commercial Medline IR systems were compared, including WinSpirs. ${ }^{2}$ Various subsets of Medline were used as document set by the different systems. For WinSpirs, two document sets were used. The systems were compared in two ways. Firstly, 18 real searches performed by clinicians were translated to queries for the 27 systems (called librarian searches). Secondly, based on the 18 searches, 18 questions were formulated and given to 2 librarians - the original questions of the clinicians were not available. The librarians conducted searches for these questions using one system; subsequently, the searches were translated in a form that could be used by the remainder of the 27 systems (now called clinical searches). The translations were made manually, and were as close to the original as possible. For the librarian searches, Silverplatter had a high performance (ranks 1 and 4 for the two document sets). For the clinical searches, Silverplatter performed considerably worse (ranks 19 and 23). A few question marks are in order. On different systems one expects to have different searches for the same questions, caused by the idiosyncrasies of the particular systems. This is ignored in this experiment because a search that was performed on one particular system is 'mapped' to multiple systems. Furthermore, a comparison of system effectiveness that is based on different document sets is dubious.

\footnotetext{
${ }^{1}$ Actually, the paper reports that the Silverplatter system was used. The Silverplatter company markets several products (Spirs, WinSpirs, WebSpirs), which are functionally roughly equivalent but have different user interfaces.

${ }^{2}$ Again, only Silverplatter was mentioned, not the name of the product.
} 
How general are the results and conclusions of the ANS evaluation? Firstly, we consider the size of the document set. Subsection 5.1.4 already reported that the results of an IR experiment depend on the size of the document set. Since the document set used for the evaluation consisted of 36,000 documents, we have to restrict our conclusions to document sets of this magnitude. It is questionable whether ANS is usable with a document set of millions of documents. This is partly due to shortcomings of the system, and partly due to the nature of searching by browsing. We elaborate on this subject in Section 7.4.

Secondly, we consider the domain of the documents and the questions. The topics of the questions had no special characteristics that distinguish them from other medical topics. Therefore, we expect that the results can be generalized to the entire medical domain. For other domains the results may be different. Because the central point of ANS is its representation of domain knowledge, we expect ANS to perform similarly in domains that are similar in size and structure to the medical domain. The questions were quite specific. This was to ensure that the test persons 'experienced' the same information need. For less articulate information needs, the browsing character of ANS may well be more advantageous. Therefore, the results pertain to specific information needs only.

Thirdly, we consider the users. The test persons in the evaluation were students. Most probably the results cannot be generalised to clinicians; the questions used are different from typical clinical questions, and the domain knowledge and search experience of clinicians also differ. Students have considerably less experience in reading primary literature than clinicians because they usually read textbooks. In Section 1.1 we illustrated the importance of IR with three examples: scientists who need scientific literature for writing publications, clinicians who have questions regarding clinical cases, and students who need information for answering questions and for writing theses. In our opinion, ANS is suitable for all these purposes. However, the experiment only covered the third group.

\subsubsection{Performance of ANS}

How does ANS perform compared to other systems?

In Chapter 6 the results of the evaluation were presented. In the overall comparison the systems turned out to be equally effective. However, for one individual question ANS was more effective, and also for the second-year students one may argue that ANS was more effective. For the second-year students, ANS was more efficient and for the fourth-year students WinSpirs was more efficient. In the overall comparison the test persons were equally satisfied with ANS and WinSpirs. However, the fourth-year students were more satisfied with WinSpirs than with ANS. The advantage of ANS over WinSpirs in terms of effectiveness, efficiency, and user satisfaction is greater for second-year students than for fourth-year students.

On the one hand, it is disappointing that the differences between the two systems are so small. On the other hand, we must bear in mind that ANS is an unpolished, 
experimental system and WinSpirs is a neat, commercial system. A commercial version of ANS is likely to be better than the current version. Additionally, the users had some experience with (Win)Spirs.

Apart from the relative effectiveness of ANS, also the absolute effectiveness of both systems was disappointing. A possible explanation for the low recall is that students are not really used to read primary literature; they acquire most of their information from textbooks. However, one would expect to find also low precision, which was not the case. How does the effectiveness compare with effectiveness reported in the literature? Shaw Jr. et al. (1997) surveyed over 100 IR papers and found that recall and precision were rarely greater than 0.50 . For the Boolean model, the average value for Rijsbergen's measure was $0.77 .^{3}$ The same value was also found in the experiment of Chapter 6. Compared to the other experiments, the precision in our experiment was high. The difference is caused by the way the retrieved set was determined: in other experiments the selection is made automatically. In our experiment the user selected the documents, which in principle includes a relevance assessment by the user. A possible cause of the low recall is the level of difficulty of the questions. Finally, it should be emphasized that results across IR experiments are difficult to compare.

In Section 1.4 we claimed that ANS' intuitive interface would reduce the procedural knowledge required to use the system. For the early versions of ANS this was certainly true, but as ANS improved, it also became more complicated. A professional user-interface designer would certainly be able to make some features more intuitive, but the number of features that are really necessary to use the system would not be reduced. During the evaluation the users did not use all of ANs (and WinSpirs) features. In fact, not all features were explained, in order not to overload the users' memory. It is a well-known fact that users of complicated systems do not use all available features; as Carroll and Olson (1988) put it: "There are some things about a system that most users never learn."

Associated with intuitiveness of the user interface is the cognitive load: the more the user has to keep in mind about the state of the system and of his search, the more difficult it will be to execute his task. Browsing generally imposes a higher cognitive load on the user than search, therefore on this aspect ANS is more demanding on the user than WinSpirs. Another issue inherent to the ANS approach is the homogeneity problem (Nielsen, 1990a). This problem occurs when browsing hypertext instead of paper documents; because the information on the screen looks homogeneous, it is difficult for the user to recognize whether he has visited a particular location or not. The same happens in ANS: by browsing meta-information instead of information the items that the user sees are more homogeneous, which makes orientation for the user more difficult.

ANS is a versatile system: in Section 4.2 we used ANS as a 'regular' IR system, as a Web browser, for classification, and as an expert system explanation facility. The

\footnotetext{
${ }^{3}$ Actually, a value of 0.23 for the harmonic mean of recall and precision is reported. This mean is equivalent with $1-$ Rijsbergen's measure $(\alpha=0.5)$.
} 
price we paid for such a general system is that it is not tailored to a specific application. For instance, WinSpirs shows the key terms of a full-text query highlighted in the retrieved documents. This is not possible with ANS, since it uses external viewers (DAPS). Thus, the possibilities for integrated document viewing are limited. In this respect, WinSpirs offers better browsing than ANs!

\subsection{General Conclusion}

In Section 1.5 we formulated two hypotheses. The first one was: The metainformation to be browsed can be represented in an Information Network: a structure that contains references to information, combined with a domain representation. This hypothesis was tested in Chapters 3 and 4 . An IR system based on the Information Network was built. Hence, the hypothesis cannot be rejected. The innovation of ANS is that it represents documents and domain in one representation in a relatively unconstrained way, and that the representation can be browsed both in its entirety and in separate layers (by applying filters).

The second hypothesis was: Search by browsing the Information Network has a higher performance than search with existing search programs. This hypothesis was tested in Chapters 5 and 6 . In a user evaluation the performance of ANS was compared with a well-known IR system. The effectiveness of ANS was slightly better, the efficiency the same, and the user satisfaction for ANS was slightly worse. If we combine these three quantities into one: performance, we must conclude that the systems perform equally well. Hence, the hypothesis must be rejected. The conclusions hold for students with specific questions about medium-sized document sets (i.e., tens of thousands of documents) from medicine and comparable domains.

Now we can address the problem statement: Is it possible to provide facilities for searching various information items by allowing the user to browse in a graphical way through meta-information in such a way that it is an improvement over existing methods? The first part of the problem statement is answered by an experimental proof: it is possible and this has been demonstrated. The second part is answered with a careful 'no, however'. Although the experiment did neither show a significantly better performance for ANS as compared to WinSpirs, nor the reverse, we must bear in mind (1) the restrictions to the conclusions mentioned in the previous paragraph, (2) the reservations we have concerning the suitability of UMLS (Subsection 7.2.2), (3) that ANS, as opposed to WinSpirs, is not a fully-developed commercial product, and (4) that the short time the test persons had to become acquainted with the systems was disadvantageous for ANS (Subsection 6.6.8).

We list the strong points of ANS:

- Usage of domain knowledge during search is encouraged because the domain knowledge is the central part of the system. In other IR systems domain knowledge is either available to the user on request or hidden from the user by means of a search intermediary. 
- The combination of browse-oriented search and availability of domain knowledge makes ANS very suitable for users who have a vague information need.

- Metabrowsing, as realised in ANS, takes browsing to a higher level. Thus, a better overview is possible of the available information and of how the information is organized.

- ANS is a versatile system; it can be used for various purposes.

We consider the following results of this research to be of general importance:

- A concrete basis on which IR systems can be compared has been given.

- In this age of electronic information it is difficult to give a good definition of the notion of document. A definition that was suitable for the ARCHIMEDES project was formulated, and several document types and properties were identified.

- The UMLS thesaurus can be used as the medical-domain-knowledge part of a medical-literature Information Network. However, it is not optimal.

- Metabrowsing is a conceivable IR model for small and medium-sized document sets.

- The success of metabrowsing depends largely on the quality of the way the meta-information is depicted and the aids to navigate through it.

- The application of ANS is not limited to IR, but can be considered as a general approach to visualizing and browsing large graphs.

Seeing that the research has resulted in an interesting, versatile system which has ample possibilities for improvements, we propose to continue this line of research.

\subsection{Future Research}

The evaluation of ANS identified several weak points of ANS. The most important complaints of users concerned the lack of a means of combining key terms and the sometimes cluttered display. The former complaint was addressed by introducing an AND/OR-like facility. The user interface of this facility appeared to be not very intuitive. This should be improved. The latter problem can be alleviated by improving the drawing algorithm. Particularly, hierarchical relationships should be associated with hierarchical placement of nodes on the screen.

In Subsection 7.2.2 we concluded that UMLS is not the perfect thesaurus for ANS. As for the medical domain, it is nevertheless the best alternative available. Figure 7.1 illustrates the problems that arise from the thesaurus topologies. Figure 7.1(a) depicts the tree structure of the MesH thesaurus, which is used to index the Medline 


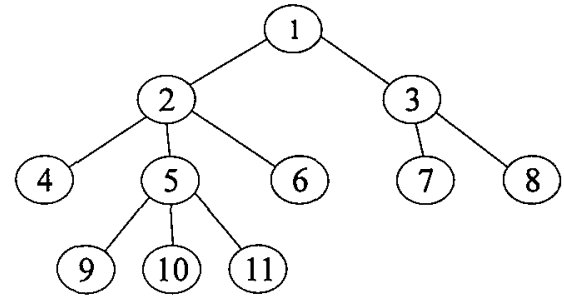

(a)

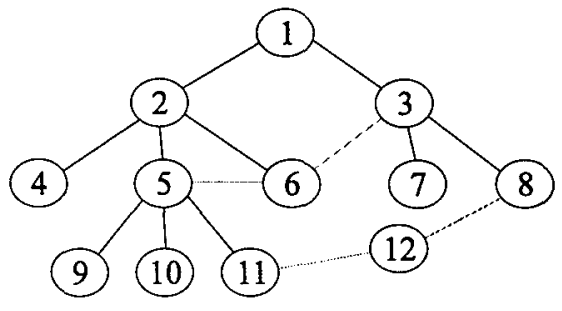

(b)

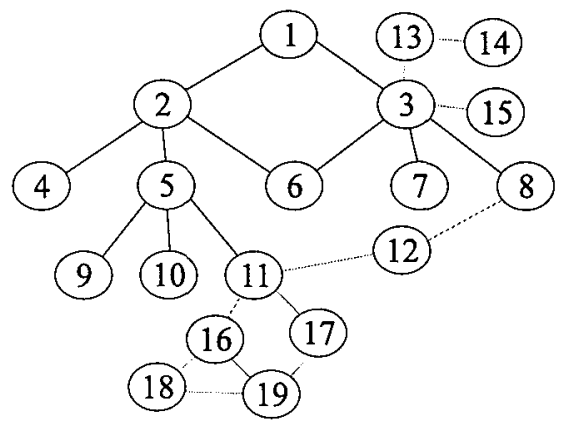

(c)

Figure 7.1: Network topologies. a: MesH thesaurus. b: UMLS thesaurus as it should be for ANS. c: Actual UMLS thesaurus as used in ANS.

documents. With the choice of UMLS instead of MesH, we expected that UMLS, being a superset of MeSH, would have more links (in (b): between 3 and 6 , and between 5 and 6) and more concepts (in (b): 12), thus creating a tangled hierarchy. This would offer the user extra possibilities to locate interesting concepts. However, on the one hand UMLS adds more concepts to MesH than we expected. These are merely confusing for the ANS user (in (c): 16-19 and 13-15). On the other hand, UMLS adds only a limited number of links to MesH (in (c): not between 5 and 6).

Instead of manually improving UMLS for our purpose this may be done at least partly in an automatic way. We discuss two of the possibilities. Firstly, uninteresting parts may be pruned. In Subsection 6.3.1 we stated that ANS' results would have been better if several very specific concepts would not have been present. For instance, the concept Transient hypertension of pregnancy has a link to Transient hypertension of pregnancy, antepartum, to Transient hypertension of pregnancy, with delivery, and to several similar concepts. These concepts have no links to documents (they cannot, because they are not MesH terms) and are, according to the link types and their specific names, more specific than Transient hypertension of pregnancy. In that case it is safe to remove these concepts because (1) they have no documents linked to 
them, (2) they are linked to the rest of the network via only one concept, and (3) as a starting point for browsing they will not be missed as long as Transient hypertension of pregnancy is not removed.

Secondly, extra links may be added. One of the shortcomings of uMLS is that several 'obvious' link types are missing. For instance, there are no links between diseases and their surgical treatments. Figure 7.2 shows an example use of such a link. Assume the user is looking for documents on the adverse effects of the various surgical treatments of the disease cholelithiasis (presence of gallstones). The only way to find Cholecystectomy adverse effects from the starting point Cholelithiasis is going to Cholelithiasis surgery, browse through the key terms of the various documents linked to it, and select the one(s) that look(s) like a surgical treatment. A direct has surgical treatment link from Cholelithiasis would be convenient in this case. To introduce such a link the following rule can be applied: if a descendant of Diseases and Injuries has a surgery subheading ${ }^{4}$ that is linked to at least $n$ documents that are also linked to a descendant of the Surgery, Operative hierarchy then a has surgical treatment link can be made from the former descendant to the latter; where $n>1$ to ensure that disease and treatment are mentioned in the same context. Figure 7.2 illustrates this rule. Here a direct link is made between the disease cholelithiasis and the surgical treatment cholecystectomy (removal of the gallbladder) since they are indirectly linked via a surgery subheading and three documents. Assume that one of the three documents also mentions a surgical treatment that has nothing to do with cholelithiasis, for instance, appendectomy (removal of the appendix). Here the $n>1$ condition prevents that a link is made between Cholelithiasis and Appendectomy. To make sure no faulty links are made, $n$ can be chosen sufficiently high. Probably more of this kind of rules can be formulated to introduce other link types. The links can be kept up to date by applying the rules every time Medline is updated.

Before making improvements to any Information Network, it is necessary to gain a better understanding of the characteristics of an Information Network that influence browsing. For instance, we observed that if a node has more neighbours than can be shown on the screen together, users are not inclined to browse through all of them. Therefore an ideal network has no nodes with more than 14 neighbours (supposing we choose 15 as maximum number of visible nodes). This can be achieved by introducing intermediate nodes.

The current ANS is not suitable for very large document sets, such as Medline, which contains over nine million documents. ${ }^{5}$ The document set of the Medline Information Network is a factor 300 smaller. This means that where now a key term has one document linked to it, in a full Medline Information Network it might have 300 documents. Naturally, it is infeasible to browse through such a number of neighbours for one node. This problem already lures in the current Medline

\footnotetext{
${ }^{4}$ The concept of subheadings was explained in Subsection 4.2.1.

${ }^{5}$ At the end of 1997 Medline contained 9.6 million documents. Approximately 33,000 new documents are added each month.
} 


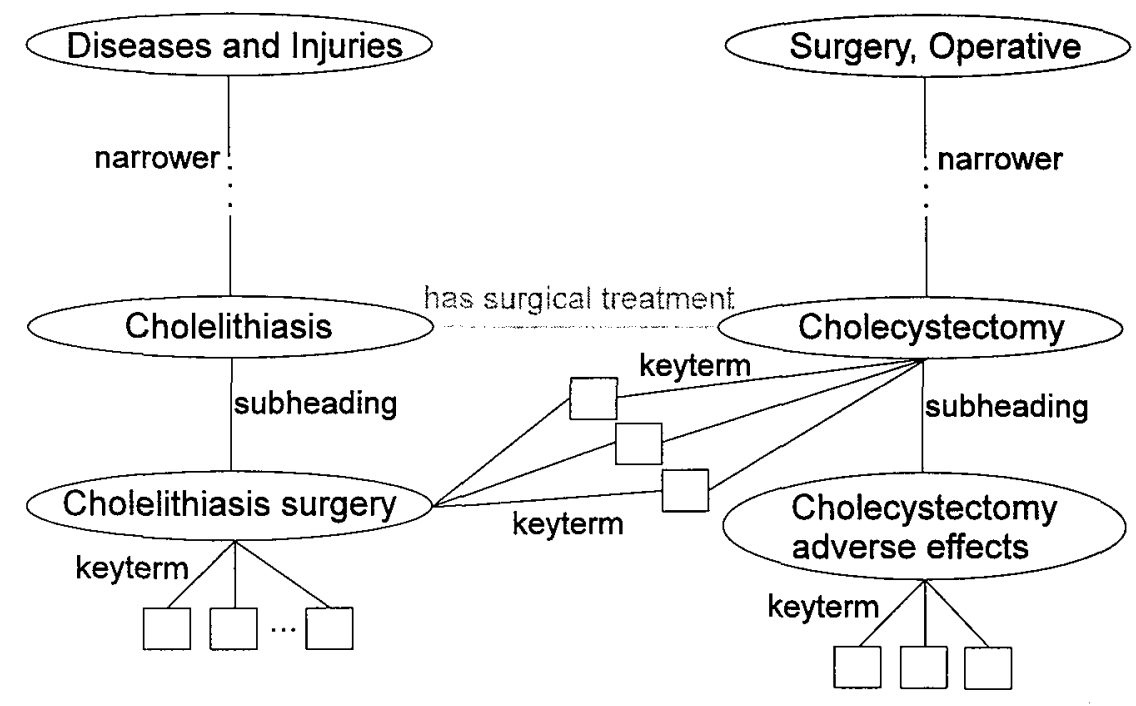

Figure 7.2: Automatic generation of has surgical treatment links.

Information Network. An extreme example is the concept Rats, which has 3496 documents linked to it.

There are at least two ways to approach the problem of very large Information Networks. Firstly, the document nodes linked to a particular concept can be shown in order of relevance to the concept. This is a form of ranked output. The ranking algorithm can estimate the relevance of the documents using the frequency of the concept in each document.

Secondly, the browse character of the search can be reduced. This can either be achieved by extending the combination facility or by reviving the query tool. The former solution has the advantage that the visual interface and the browsing of meta-information is, to a certain extent, retained. For the latter solution document nodes are not necessary any more in the Information Network. The browser could still be used in what Lesk and Salton (1971) call the presearch: locating the concepts that are relevant to the information need before the actual queries are formulated. A consequence of removing document nodes from the Information Network is that browsing from concept to document to concept (as described in Subsection 6.3.1) is not possible anymore. This can be circumvented by introducing a new link type that links concepts together that have at least one document in common.

Furthermore, it is not essential to be able to search the entire Medline. Greschner et al. (1996) reports that only in $3 \%$ of clinical searches the users want to search all years present in Medline; the majority of the searches concerns the last 3 to 5 years. Limiting the document set to such a period would reduce the document 
set size with approximately a factor 2 or 3 . When a Medline document set on a single CD-ROM is concerned, it contains a subset anyhow.

In Section 1.1 the importance of IR in clinical practice was discussed. An IR system in a clinical context can be even more useful if it is connected with other systems. Rada et al. (1992) describe a prototype system that integrates an electronic patient record with Embase, a medical literature IR system similar to Medline (but smaller). An intelligent search intermediary constructs Embase queries based on information from a particular patient record. Instead of an electronic patient record Miller et al. (1991) connect a diagnostic decision-support system to a medical literature IR system. The idea is that once a diagnosis has been established, relevant literature can be retrieved automatically. A Medline query is predefined for each disease in the knowledge base. Users can also edit the query to tailor it to their needs. ANs could be used in a similar way: information obtained from an electronic patient record or a decision-support system diagnosis can serve as a starting point for browsing with ANS. This is similar to the transnational income tax expert system that was connected to ANS (Subsection 4.2.4). Supplying only a concept as starting point is not sufficient; a possible improvement would be the possibility to pass a (Boolean) query to ANs. The results can be browsed in the same way as results of the combination facility (as described in Subsection 4.4.4, in particular Figure 4.7).

The experiment with ANS and the Web (Subsection 4.2.2) was small, but it was interesting enough to be continued on a larger scale. As the user browses with his Web browser, the URLs can be passed on to ANS and stored in the Information Network. This requires ANS to be extended such that the Information Network can be updated in real time. Also an indexing mechanism must be added. The bottleneck is, naturally, the domain representation. Because the domain of the Web is unlimited, a global domain representation is required. An attempt to such a representation is WordNet. However, mixed successes have been reported with the application of WordNet in IR (see Subsection 2.3.2). Another approach would be to restrict the system to a particular domain. For medicine, for instance, uMLS could be used. The great challenge in building the Information Network for the metaWeb-browser concerns the management of the plethora of hyperlinks. A typical Web home page may contain dozens of them. A sophisticated algorithm is required that ignores uninteresting links (e.g., commercial banners) and assigns link types and node types. The users' successful usage of filters depends on the number of sensible link and node types that the algorithm can discern.

Several meta-Web-browsers exist. Cockburn and Jones (1997) discuss twelve of them. Various ways of vizualizing the Web are applied; graph-based, tree-like and cluster-based views are used. The majority of the interfaces is two dimensional, some are three dimensional. Some systems use static (i.e., precomputed) views, others, like ANS, generate the view while the user browses. None of the systems includes domain knowledge. A few systems have some kind of filter mechanism. Apart from 
link type and node type filters, two other filters are used. Interaction-based filters are based on the history of the user's navigational actions. For instance, one can filter nodes that have been visited over the last week. Structure-based filters are based on the topology of the visualization. One can consider ANs' algorithm as using one: when more than a particular maximum of nodes is about to be shown, the nodes that are the most distant from the last accessed node are filtered.

To summarize, future research should be directed at improving the ANs browser, the domain representation in general, and particularly the domain representation for medical Information Networks. Finally, ANS has a wide applicability by its further integration into other information systems. 



\section{Appendix}

\section{Questions for the Test Persons}

Potential question subjects for the evaluation of ANS were identified by selecting subjects from the third-year and fourth-year curriculum that were not discussed in the first-year and second-year curriculum. For these subjects a rough estimate was made (using ANS and WinSpirs) how many documents were present on the Medline CD-ROM. For the following subjects ample documents were present: obstetrics, airways disorders, liver cirrhosis, anemia, malaria, epilepsy, fractures. Eventually the subjects obstetrics, airways disorders, and fractures were chosen because of their diversity and the availability of experts.

It was considered to take questions that had arisen from cases discussed in small educational groups ${ }^{1}$, but this idea was discarded because the formulation of the questions was not sufficiently precise and because it would be very difficult to find good questions for which relevant documents existed on the Medline CD-ROM.

Three experts, who also participated in the assessment of the search results, each composed a question in consultation with the experimenter. The questions were formulated in Dutch whereas ANS and WinSpirs use English. Thus, it was made harder for the test persons to extract relevant key terms from the questions. During the pre-test with five test persons small changes were made in the phrasing of the questions.

The test persons were instructed to find as many relevant documents as possible, and as little irrelevant documents as possible. They had to stop searching as soon as they thought they were finished. It was explained that the search was constrained to a subset of Medline.

\footnotetext{
${ }^{1}$ The educational system used at Maastricht University is called problem-based learning. Students discuss problems (cases) in small educational groups according to a structured procedure. Part of the procedure is the formulation of learning goals, which are questions (formulated by the students) that the group will try to answer by reading and discussing literature.
} 


\section{Question A}

The first question concerned airways disorders. This subject is part of the third year. The question was:

Find documents about the effects of different kinds of smoke on the respiratory system.

\section{Question B}

The second question concerned bone fractures. This subject is briefly introduced in the first year, but the treatment of fractures, which is the subject of the question, is dealt with in the fourth year. The question was:

Find documents about materials which a doctor leaves - on purpose in the human body when fixating fractures. Objects that are accidentally left behind are not intended.

At the moment it was formulated this was thought to be a question on a relative simple subject.

\section{Question C}

The third question concerned eclampsia (hypertension during pregnancy). This subject is part of the third and fourth year. The question was:

Find documents about complications with eclampsia and eclampsiarelated problems for the mother.

At the moment it was formulated this was thought to be a difficult question on a difficult subject. The notion of 'eclampsia-related' is of course ambiguous: different people will have a different idea of what is and what is not related to eclampsia. 


\section{References}

Aigrain P. and Longueville V. (1991). A connection graph for user navigation in a large image bank. Proceedings of a Conference on Intelligent Text and Image Handling 'RIAO 91' (ed. A. Lichnerowicz), pp. 67-85, Elsevier, Amsterdam, The Netherlands. (17)

Amrine F. and Zucker F.J. (1987). Introduction. Goethe and the Sciences: A Reappraisal (eds. F. Amrine, F.J. Zucker, and H. Wheeler), Vol. 97 of Boston Studies in the Philosophy of Science, pp. xi-xv. D. Reidel Publishing Company, Dordrecht, The Netherlands.

B3 SKBS (1991). B3-project: Multi-media knowledge-based systems. Project proposal, Delft University of Technology/University of Limburg.

Baron L., Tague-Sutcliffe J., Kinnucan M.T., and Carey T. (1996). Labeled, typed links as cues when reading hypertext documents. Journal of the American Society for Information Science, Vol. 47, No. 12, pp. 896-908. (50)

Bayes T. (1763). An essay toward solving a problem in the doctrine of chance. Philosophical Transactions of the Royal Society of London, Vol. 53, pp. 370418. Reprinted in Biometrika 45, 1958, pp. 296-315.

Belkin N.J. (1981). Ineffable concepts in information science. Information Retrieval Experiment (ed. K. Sparck Jones), Chapter 4, pp. 44-58. Butterworths, London, England. $(92,135)$

Berners-Lee T., Cailliau R., Groff J.F., and Pollermann B. (1992). World-Wide Web: The information universe. Electronic Networking: Research, Applications and Policy, Vol. 2, No. 1, pp. 52-58. (36)

Berners-Lee T., Masinter L., and McCahill M. (1994). Uniform resource locators (URL). Request For Comments (Proposed Standard) RFC 1738, Internet Engineering Task Force. $(51,66)$ 
Berners-Lee T. and Connolly D. (1995). Hypertext Markup Language - 2.0. Request For Comments (Proposed Standard) RFC 1866, Internet Engineering Task Force. (72)

Blair D.C. and Maron M. (1985). An evaluation of retrieval effectiveness for a fulltext document-retrieval system. Communications of the ACM, Vol. 28, No. 3, pp. 289-299. (3, 49, 91)

Blair D.C. (1996). STAIRS redux: Thoughts on the STAIRS evaluation, ten years after. Journal of the American Society for Information Science, Vol. 47, No. 1, pp. $4-22$.

Bodner R.C. and Song F. (1996). Knowledge-based approaches to query expansion in information retrieval. Lecture Notes in Computer Science, Vol. 1081, pp. $146-158 . \quad(6,21,27,28,35)$

Borenstein N.S. (1992). Internet multimedia mail with MIME: Emerging standard interoperability. Proceedings of ULPAA '92.

Bourdoncle F. (1997). LiveTopics: recherche visuelle d'information sur l'Internet. Computer-Assisted Information Searching on Internet (RIAO'97), pp. 651-654, Montreal, Canada.

Bowman C.M., Danzig P.B., Hardy D.R., Manber U., and Schwartz M.F. (1994). Harvest: A scalable, customizable discovery and access system. Technical Report CU-CS-732-94, University of Colorado, Department of Computer Science. (36)

Briet S. (1951). Qu'est-ce que la Documentation. EDIT, Paris, France.

Brockhaus (1969). Brockhaus Enzyklopädie, Vol. 7. F.A. Brockhaus, Wiesbaden, Germany, 17th edition.

Buckland M.K. and Gey F. (1994). The relationship between recall and precision. Journal of the American Society for Information Science, Vol. 45, No. 1, pp. 12-19. $(16,89)$

Buckland M.K. (1997). What is a "document"? Journal of the American Society for Information Science, Vol. 48, No. 9, pp. 804-809.

Callan J.P., Croft W.B., and Harding S.M. (1992). The INQUERY retrieval system. Proceedings of the Third International Conference on Database and Expert Systems Applications (eds. A.M. Tjoa and I. Ramos), pp. 78-83. (22, 27, 29, $31,32,35)$

Campbell B. and Goodman J.M. (1988). HAM: A general purpose hypertext abstract machine. Communications of the ACM, Vol. 31, No. 7, pp. 856-861. 
Can F. (1993). Incremental clustering for dynamic information processing. ACM Transactions on Information Systems, Vol. 11, No. 2, pp. 143-164. (21, 36)

Carmel E., Crawford S., and Chen H. (1992). Browsing in hypertext: A cognitive study. IEEE Transactions on Systems, Man, and Cybernetics, Vol. 22, No. 5, pp. $865-884$.

Carroll J.M. and Olson J.R. (1988). Mental models in human-computer interaction. Handbook of Human-Computer Interaction (ed. M. Helander), Chapter 2. North-Holland, Amsterdam, The Netherlands. $(5,138)$

Cavnar W.B. (1995). Using an N-gram-based document representation with a vector processing retrieval model. Overview of the Third TExt Retrieval Conference (TREC-3) (ed. D.K. Harman), National Institute of Standards and Technology, Gaithersburg, MD. (18)

Chambliss M.L. and Conley J. (1996). Answering clinical questions. The Journal of Family Practice, Vol. 43, No. 2, pp. 140-144.

Chang S. and Rice R.E. (1993). Browsing: A multidimensional framework. Annual Review of Information Science and Technology, Vol. 28, pp. 231-275.

Charoenkitkarn N., Chignell M., and Golovchinsky G. (1995). Interactive exploration as a formal text retrieval method: How well can interactivity compensate for unsophisticated retrieval algorithms. Overview of the Third TExt Retrieval Conference (TREC-3) (ed. D.K. Harman), National Institute of Standards and Technology, Gaithersburg, MD. $(18,27,28,30,31,35)$

Chen H., Lynch K.J., Basu K., and Ng T. (1993). Generating, integrating, and activating thesauri for concept-based document retrieval. IEEE Expert, Vol. 8, No. 2, pp. 25-34. $\quad(24,27,28,31,32,35)$

Chen H., Schatz B., Ng T., Martinez J., Kirchhoff A., and Lin C. (1996). A parallel computing approach to creating engineering concept spaces for semantic retrieval: The Illinois Digital Library Initiative project. IEEE Transactions on Pattern Analysis and Machine Intelligence, Vol. 18, No. 8, pp. 771-782.

Chu H. and Rosenthal M. (1996). Search engines for the World Wide Web: a comparative study and evaluation. Methodology in Global complexity: information, chaos and control: ASIS annual meeting 1996 (ed. S. Hardin), pp. 127-135. (39)

Cockburn A. and Jones S. (1997). Design issues for World Wide Web navigation visualisation tools. Computer-Assisted Information Searching on Internet (RIAO'97), pp. 55-74, Montreal, Canada. 
Collier G.H. (1987). Thoth-II: Hypertext with explicit semantics. HYPER87 Proceedings of ACM Hypertext'87 Conference (eds. S. Weiss and M. Schwartz), pp. 269-289, ACM Press, Chapel Hill, NC. (50, 132)

Conklin J. and Begeman M.L. (1988). gIBIS: A hypertext tool for exploratory policy discussion. ACM Transactions on Office Information Systems, Vol. 6, No. 4, pp. $303-331 . \quad(50,77)$

Consens M. and Medezon A. (1989). Expressing structural hypertext queries in Graphlog. HYPER89 Proceedings of ACM Hypertext'89 Conference (eds. F. Halasz and N. Meyrowitz), pp. 269-292, ACM Press, Pittsburgh, PA. (18, $30,32,33,50)$

Cove J.F. and Walsh B.C. (1988). Online text retrieval via browsing. Information Processing \& Management, Vol. 24, No. 1, pp. 31-37. (8, 9, 10)

Croft W.B. and Thompson R.H. (1987). $I^{3} R$ : A new approach to the design of document retrieval systems. Journal of the American Society for Information Science, Vol. 38, No. 6, pp. 389-404.

Crouch C.J. and Yang B. (1992). Experiments in automatic statistical thesaurus construction. Proceedings of the 15th Annual International ACM/SIGIR Conference on Research and Development in Information Retrieval (eds. N. Belkin, P. Ingwersen, and A. Mark Pejtersen), pp. 77-88, ACM Press, New York, NY. (27)

Crouch D., Crouch C.J., and Andreas G. (1989). The use of cluster hierarchies in hypertext information retrieval. HYPER89 Proceedings of ACM Hypertext'89 Conference (eds. F. Halasz and N. Meyrowitz), pp. 225-237, ACM Press, Pittsburgh, PA. $\quad(18,20,24,29,31,33,36)$

Date C.J. (1990). An Introduction to Database Systems, Vol. I. Addison-Wesley Publishing Company, Reading, MA. (47, 53)

De Bra P.M.E., Houben G.J., and Kornatzky Y. (1992). An extensible data model for hyperdocuments. ECHT92 Proceedings of ECHT'92 the Fourth ACM Conference on Hypertext (eds. D. Lucarella, J. Nanard, M. Nanard, and P. Paolini), pp. 222-231, ACM Press, New York, NY. $(44,45)$

Di Battista G., Eades P., Tamassia R., and Tollis I.G. (1994). Algorithms for drawing graphs: An annotated bibliography. Computational Geometry: Theory and Applications, Vol. 4, pp. 235-282.

Dietz J.L.G. (1994). A universal, ontology-free conceptual modelling technique. Technical report, Delft University of Technology. Research Memorandum. 
Dietz J.L.G., Van der Pol R., and Wiesman F. (1997). The ARCHIMEDES Network System: a system for searching and accessing information in multiple multimedia sources. Journal of Intelligent Information Systems, Vol. 8, No. 1, pp. 77-101. Also published as report SKBS/B3.A/94-08.

Eades P. (1984). A heuristic for graph drawing. Congressus Numerantium, Vol. 42, pp. $149-160 . \quad(59,78)$

Fielding R.T. (1994). Maintaining distributed hypertext infostructures: welcome to MOMspider's Web. Computer Networks and ISDN Systems, Vol. 27, No. 2, pp. 193-204.

Fielding R.T. (1995). Relative Uniform Resource Locators. Request For Comments (Proposed Standard) RFC 1808, Internet Engineering Task Force. (51, 66)

Findler N., Maini S., and Yuen A.F.M. (1992). SHRIF, a general-purpose system for heuristic retrieval of information and facts, applied to medical knowledge processing. Information Processing \& Management, Vol. 28, No. 2, pp. 219240. $(28,29,32,36)$

Frants V.I., Shapiro J., and Voiskunskii V.G. (1996). Development of IR systems: new direction. Information Processing \& Management, Vol. 32, No. 3, pp. 373386 .

Furuta R. and Stotts P.D. (1990). The Trellis hypertext reference model. Proceedings of the Hypertext Standardization Workshop by National Institute of Science and Technology (NIST), National Institute of Standards and Technology, Gaithersburg, MD.

Garzotto F., Paolini P., and Schwabe D. (1991). HDM - a model for the design of hypertext applications. HYPER91 Proceedings of ACM Hypertext'91 Conference (ed. J. Walker), pp. 313-328, ACM Press, San Antonio, TX.

Gauch S. and Smith J.B. (1991). Search improvement via automatic query reformulation. ACM Transactions on Information Systems, Vol. 9, No. 3, pp. 249-280. $(18,26,27,28,31,35)$

Glushko R. (1989). Design issues for multi-document hypertexts. HYPER89 Proceedings of ACM Hypertext'89 Conference (eds. F. Halasz and N. Meyrowitz), pp. 51-60, ACM Press, Pittsburgh, PA. (44)

Gordon M. (1988). Probalistic and genetic algorithms for document retrieval. Communications of the ACM, Vol. 31, No. 10, pp. 1208-1218. (21,34,35)

Gosling J. and McGilton H. (1995). The Java(tm) language environment: A white paper. Technical report, Sun Microsystems. $(62,74)$ 
Greschner M., Rassweiler J.J., Weber A., and Alken P. (1996). New methods of searching for medical literature: Comparison of two systems, implementation and user reactions in a urologic department. Urologia Internationalis, Vol. 57, No. 1, pp. 1-4. $(136,143)$

Gupta A. and Jain R. (1997). Visual information retrieval. Communications of the $A C M$, Vol. 40, No. 5, pp. 70-79.

Haan B.J., Kahn P., Riley V.A., Coombs J.H., and Meyrowitz N.K. (1992). IRIS hypermedia services. Communications of the ACM, Vol. 35 , No. 1, pp. 36-51. (44)

Hage J., Van der Meulen M., and Span G. (1993). Intelligent information retrieval from multiple databases. Informatica e Diritto, , No. 3, pp. 149-164. Also published as report SKBS/B3.A/92-10. (41)

Hahn U. and Reimer U. (1988). Automatic generation of hypertext knowledge bases. ACM SIGOIS Bulletin, Vol. 9, No. 2-3, pp. 182-188.

Halasz F.G., Morgan T., and Trigg R. (1987). Notecards in a nutshell. Proceedings of the 1987 ACM Conference of Human Factors in Computer Systems, pp. 45-52. (50)

Halasz F.G. and Schwartz M.D. (1990). The Dexter hypertext reference model. Proceedings of the NIST Hypertext Standardization workshop, pp. 95-133, NIST Special Publication 500-178, Gaithersburg, MD. $\quad(44,45)$

Halasz F.G. and Schwartz M.D. (1994). The Dexter hypertext reference model. Communications of the ACM, Vol. 37, No. 2, pp. 30-39. (50)

Harman D.K. (1992). Ranking algorithms. Information Retrieval: Data Structures \& Algorithms (eds. W.B. Frakes and R. Baeza-Yates), Chapter 14, pp. 363-391. Prentice Hall, Englewood Cliffs, NJ. (34)

Harman D.K. (1994). Overview of the second text retrieval conference (TREC-2). The Second Text REtrieval Conference (TREC-2) (ed. D.K. Harman), pp. 1-20, National Institute of Standards and Technology, Gaithersburg, MD.

Harman D.K. (1995). Overview of the third text retrieval conference (TREC-3). The third text retrieval conference (TREC-3) (ed. D.K. Harman), pp. 1-19, National Institute of Standards and Technology, Gaithersburg, MD.

Harter S.P. (1996). Variations in relevance assessments and the measurement of retrieval effectiveness. Journal of the American Society for Information Science, Vol. 47, No. 1, pp. 37-49. 
Hawkins D.T. (1996). Hunting, grazing, browsing: A model for online information retrieval. Online, Vol. 20, No. 1, pp. 71-73. (10)

Haynes R.B., Wilczynski N., McKibbon K.A., Walker C.J., and Sinclair J.C. (1994). Developing optimal search strategies for detecting clinically sound studies in MEDLINE. Journal of the American Medical Informatics Association, Vol. 1, No. 6, pp. 447-458. (136)

Hemmje M., Kunkel C., and Willett A. (1994). Lyberworld - A visualization user interface supporting fulltext retrieval. Proceedings of the Seventeenth Annual International ACM SIGIR Conference on Research and Development in Information Retrieval (eds. W.B. Croft and C.J. van Rijsbergen), pp. 249-259, Springer-Verlag, London, England. (50, 132)

Hersh W.R., Buckley C., Leone T.J., and Hickam D. (1994). OHSUMED: An interactive retrieval evaluation and new large test collection for research. Proceedings of the Seventeenth Annual International ACM SIGIR Conference on Research and Development in Information Retrieval (eds. W.B. Croft and C.J. Van Rijsbergen), pp. 192-201, Springer-Verlag, London, England.

Howard H. (1992). Measures that discriminate among online searchers with different training and experience. Online Review, Vol. 6, pp. 315-327.

Hristovski D., Rožić A., and Dimec J. (1994). Unified access to medical bibliographic databases. Proceedings of the Twelfth International Congress of the European Federation for Medical Informatics (MIE'94) (eds. P. Barahona, M. Veloso, and J. Bryant), pp. 517-522.

Humphrey S. (1992). Indexing biomedical documents: From thesaural to knowledgebased retrieval systems. Artificial Intelligence in Medicine, Vol. 4, No. 5, pp. 343-371. $(18,27,28,32)$

ISO (1986). Documentation - guidelines for the establishment and development of monolingual thesauri. Technical Report ISO 2788, International Organisation for Standardisation, Geneva, Switzerland. $\quad(27,65)$

ISO/IEC (1989). ISO 8613, text \& office systems - office document architecture (ODA) and interchange format. Technical report, OSO/IEC, Geneva, Switzerland. (47)

ISO/IEC (1993). MHG WD S7. Information technology - coded representation of multimedia and hypermedia information objects. Part 1: Base notation. Technical Report N 235, OSO/IEC, Geneva, Switzerland.

Jansen R. (1993). Thesaurusrelationen als instrumentelle Hilfsmittel für Hypertext und Wissensbanken? Nachrichten für Dokumentation: Zeitschrift für Information und Dokumentation, Vol. 44, pp. 7-14. 
Jing Y. and Croft W.B. (1994). An association thesaurus for information retrieval. Technical report UMass 94-17, University of Massachusetts, Amherst.

Kent A., Berry M., Leuhrs F.U., and Perry J.W. (1955). Machine literature searching VIII. Operational criteria for designing information retrieval systems. American Documentation, Vol. 6, No. 2, pp. 93-101. (16,89)

Klein M.S., Vantoll Ross F., Adams D.L., and Gilbert C.M. (1994). Effect of online literature searching on length of stay and patient care costs. Academic Medicine, Vol. 69 , No. 6 , pp. $489-495$.

Krovetz R. and Kroft W.B. (1992). Lexical ambiguity and information retrieval. ACM Transactions on Information Systems, Vol. 10, No. 2, pp. 115-141.

Kwok K.L. (1990). Experiments with a component theory of probabilistic information retrieval based on single terms as document components. ACM Transactions on Information Systems, Vol. 8, No. 4, pp. 363-386. (22, 28, 31, 35)

Landis J.R. and Koch G.G. (1977). The measurement of observer agreement for categorical data. Biometrics, Vol. 33, No. 1, pp. 159-174. (101)

Layaida R. and Caron A. (1994). Application of the backpropagation algorithm to an information retrieval system. RIAO 94 Conference Proceedings - Intelligent Multimedia Information Retrieval Systems and Management, pp. 161-171, Centre de Hautes Etudes Internationales d'Informatique Documentaire, Paris, France. $(24,27,28,29,31,34,35)$

Lee J.H. (1994). Properties of extended Boolean models in information retrieval. Proceedings of the Seventeenth Annual International ACM-SIGIR Conference on Research and Development in Information Retrieval (SIGIR'94) (eds. W.B. Croft and C.J. van Rijsbergen), pp. 182-190, Springer-Verlag, London, England. (32)

Lenman S., See H., Century M., and Pennycook B. (1996). Merz: Creating personal and shared spaces on the World Wide Web. Webnet '96 - World Conference of the Web Society Proceedings (ed. H. Maurer), pp. 292-297, Association for the Advancement of Computing in Education, Charlottesville, VA.

Lesk M.E. and Salton G. (1971). Interactive search and retrieval methods using automatic information displays. The SMART Retrieval System (ed. G. Salton), Chapter 25. Prentice Hall, Englewood Cliffs, NJ. (143)

Lindberg D.A.B., Humphreys B.L., and McCray A.T. (1993a). The unified medical language system. Yearbook of Medical Informatics 1993, pp. 41-51. Schattauer, Stuttgart, Germany. $(28,69)$ 
Lindberg D.A.B., Siegel E.R., Rapp B.A., Wallingford K.T., and Wilson S.R. (1993b). Use of MEDLINE by physicians for clinical problem solving. Journal of the American Medical Association, Vol. 269, No. 24, pp. 3124-3129.

Lowe H.J. and Barnett G.O. (1994). Understanding and using the medical subject headings (MeSH) vocabulary to perform literature searches. Journal of the American Medical Association, Vol. 271, No. 14, pp. 1103-1108. $(28,31)$

Lu X.A., Holt J.D., and Miller D.J. (1996). Boolean systems revisited: Its performance and its behavior. The Fourth Text REtrieval Conference (TREC-4) (ed. D.K. Harman), National Institute of Standards and Technology, Gaithersburg, MD. (20)

Lucarella D. and Zanzi A. (1994). A framework for integrating browsing and searching in hypertext systems. Informatica e diritto, Vol. 11, pp. 79-99.

Lucas P.J.F. and Van der Gaag L.C. (1991). Principles of Expert Systems. AddisonWesley, Reading, MA. (73)

Maida A.S. and Shapiro S.C. (1982). Intensional concepts in propositional semantic networks. Cognitive Science, Vol. 6, No. 4, pp. 291-330. Reprinted in R.J. Brachman and H.J. Levesque, eds. (1985) Readings in Knowledge Representation, pp. 170-189, Morgan Kaufmann, Los Altos, CA. (11, 65)

Marson A.G. and Chadwick D.W. (1996). How easy are randomized controlled trials in epilepsy to find on Medline? The sensitivity and precision of two Medline searches. Epilepsia, Vol. 37, No. 4, pp. 377-380.

McMath C., Tamaru R.S., and Rada R. (1989). A graphical thesaurus-based information retrieval system. International Journal of Man-Machine Studies, Vol. 31, pp. 121-147. $(50,61)$

Miller G.A. (1990). WordNet: An on-line lexical database. International Journal of Lexicography, Vol. 3, No. 4, pp. 235-244.

Miller G.A (1995). WordNet: A lexical database for english. Communications of the ACM, Vol. 38, No. 11, pp. 39-41. (27)

Miller R.A., Jamnback L., Giuse N.B., and Masarie Jr. F.E. (1991). Extending the capabilities of diagnostic decision support programs through links to bibliographic searching: Addition of "canned MeSH logic" to the quick medical reference (QMR) program for use with Grateful Med. Proceedings of the Fifteenth Annual Symposium on Computer Application in Medical Care, pp. 150-155, New York, NY. (144)

Mizzaro S. (1997). Relevance: The whole history. Journal of the American Society for Information Science, Vol. 48, No. 9, pp. 810-832. 
Motro A. (1988). VAGUE: A user interface to relational databases that permits vague queries. ACM Transactions on Information Systems, Vol. 6, No. 3, pp. 187-214. $\quad(21,27,32,35)$

Nielsen J. (1990a). The art of navigating through hypertext. Communications of the ACM, Vol. 33, No. 3, pp. 296-310. $\quad(50,81,138)$

Nielsen J. (1990b). Hypertext and hypermedia. Academic Press, Boston, MA.

NLM (1995). UMLS knowledge sources documentation. Technical report, National Library of Medicine. (70)

Notess G.R. (1997). On the nets. Database, Vol. 20, No. 1, pp. 61-64. (39)

Oddy R.N. (1981). Laboratory tests: automatic systems. Information Retrieval Experiment (ed. K. Sparck Jones), Chapter 9, pp. 156-177. Butterworths, London, England. (88)

Oddy R.N., Liddy E.D., Balakrishnan B., Bishop A., Elewononi J., and Martin E. (1992). Towards the use of situational information in information retrieval. Journal of Documentation, Vol. 48, No. 2, pp. 123-171.

Otlet P. (1934). Traité de Documentation. Editiones Mundaneum, Brussels, Belgium. Reprinted: 1989, Centre de Lecture Publique de la Communauté Fran caise, Liège. (43)

Parsaye K., Chignell M., Khoshafian S., and Wong H. (1989). Intelligent Databases: Object-Oriented, Deductive Hypermedia Technologies. John Wiley and Sons, New York, NY.

Rada R., Barlow J., Bijstra D., Potharst J., de Vries Robbé P., and Zansra P. (1992). OAR: Open architecture for reasoning applied to connecting patient records to medical literature. Advances in Medical Informatics (eds. J. Noothoven van Goor and J.P. Christensen), pp. 287-294. IOS Press, Amsterdam, The Netherlands. (144)

Rees A.M. (1966). The relevance of relevance to the testing and evaluation of document retrieval systems. ASLIB Proceedings, Vol. 18, No. 11, pp. 316-324. (92)

Reisner P. (1988). Query languages. Handbook of Human-Computer Interaction (ed. M. Helander), Chapter 12. North-Holland, Amsterdam, The Netherlands.

Robertson S.E. (1977). The probability ranking principle in IR. Journal of Documentation, Vol. 33, No. 4, pp. 294-304. (21) 
Robertson S.E. (1981). The methodology of information retrieval experiment. Information Retrieval Experiment (ed. K. Sparck Jones), Chapter 2. Butterworths, London, England. (15, 87, 88, 89, 91, 92, 93)

Robertson S.E. (1992). On the evaluation of IR systems. Information Processing \& Management, Vol. 28, No. 4, pp. 457-466. (88)

Robertson S.E., Walker S., Beaulieu M.M., Gatford M., and Payne A. (1996). Okapi at TREC-4. The Fourth Text REtrieval Conference (TREC-4) (ed. D.K. Harman), National Institute of Standards and Technology, Gaithersburg, MD. $(22,28,31,35)$

Roethlisberger F.J. and Dickson W.J. (1939). Management and the worker: An account of a research program conducted by the Western Electric Company, Hawthorne Works, Chicago. Harvard University Press, Cambridge, MA.

Rose D.E. and Belew R.K. (1991). A connectionist and symbolic hybrid for improving legal research. International Journal of Man-Machine Studies, Vol. 35, No. 1 , pp. $1-33 . \quad(24,28,30,31,32,34,50)$

Rose D.E. and Stevens C. (1997). V-Twin: A lightweight engine for interactive use. Information Technology: The Fifth Text REtrieval Conference (TREC5) (eds. D.K. Harman and E.M. Voorhees), National Institute of Standards and Technology, Gaithersburg, MD. $\quad(6,21,28,31,35)$

Rožić H.D. and Dimec J. (1994). Unified access to medical bibliographic databases. Proceedings of the Twelfth International Congress of the European Federation for Medical Informatics (MIE'94) (eds. P. Barahona, M. Veloso, and J. Bryant), pp. $517-522$. (62)

Sachs W.M. (1976). An approach to associative retrieval through the theory of fuzzy sets. Journal of the American Society for Information Science, Vol. 27, No. 2, pp. $85-87$.

Salton G., Fox E.A., and Wu H. (1983). Extended Boolean information retrieval. Communications of the ACM, Vol. 26, No. 11, pp. 1022-1036.

Salton G. and McGill M.J. (1983). Introduction to Modern Information Retrieval. McGraw-Hill, New York, NY. $(1,18,21,28)$

Salton G. and Buckley C. (1988). Term-weighting approaches in automatic text retrieval. Information Processing \& Management, Vol. 24, No. 5, pp. 513-523. (19)

Salton G. and Buckley C. (1990). Improving retrieval performance by relevance feedback. Journal of the American Society for Information Science, Vol. 41, No. 4 , pp. $288-297$. 
Salton G. (1991). Developments in automatic text retrieval. Science, Vol. 253, pp. 974-980. $(18,19)$

Saracevic T. (1995). Evaluation of evaluation in information retrieval. SIGIR Forum, Vol. 8, pp. 138-146.

Savoy J. and Desbois D. (1991). Information retrieval in hypertext systems: an approach using Bayesian networks. Electronic Publishing, Vol. 4, No. 2, pp. $87-108 . \quad(22,28,33,35)$

Savoy J. (1994). Searching information in legal hypertext systems. Artificial Intelligence and Law, Vol. 2, pp. 205-232. (20,22)

Schiffman S.S., Reynolds M.L., and Young F.W. (1981). Introduction to Multidimensional Scaling: Theory, Methods and Applications. Academic Press, New York, NY. $(59,78)$

Schouten H.J.A. (1995). Klinische Statistiek: Een Praktische Inleiding in Methode en Analyse. Bohn Stafleu van Loghum, Houten, The Netherlands.

Schwartz D.G. and Schwartz S.A. (1995). MEDLINE training for medical students integrated into the clinical curriculum. Medical Education, Vol. 29, pp. 133-138. (3)

Senn S. (1993). Cross-Over Trials in Clinical Research. John Wiley and Sons, Chichester, England. (109)

Shaw W. (1986). On the foundation of evaluation. Journal of the American Society for Information Science, Vol. 37, No. 5, pp. 346-348. (90)

Shaw Jr. W.M., Burgin R., and Howell P. (1997). Performance standards and evaluations in IR test collections: Vector-space and other retrieval models. Information Processing \& Management, Vol. 33, No. 1, pp. 15-36. (4, 17, 21, 91, 138)

Shneiderman B. (1992). Designing the User Interface: Strategies for Effective Human-Computer Interaction. Addison-Wesley, Reading, MA, 2nd edition. $(10,11)$

Shneier M. and Abdel-Mottaleb M. (1996). Exploiting the JPEG compression scheme for image retrieval. IEEE Transactions on Pattern Analysis and Machine Intelligence, Vol. 18 , No. 8, pp. 849-853. (17)

Smeaton A. and Rijsbergen C. (1983). The retrieval effects on query expansion on a feedback document retrieval system. Computer Journal, Vol. 26, No. 3, pp. 239-246. (27)

Smith J.M. (1988). SGML - the users's guide to ISO 8879, standard generalized markup language. Technical Report 9979-1986, ISO/IEC. 
Smith M.E. (1990). Aspects of the P-norm model of information retrieval: Syntactic query generation, efficiency, and theoretical properties. Technical Report CORNELLCS//TR90-1128, Cornell University, Computer Science Department. (32)

Smith P.J., Shute S.J., Galdes D., and Chignell M.H. (1989). Knowledge-based search tactics for an intelligent intermediary system. ACM Transactions on Information Systems, Vol. 7, No. 3, pp. 247-270. (18, 27, 28, 29, 31)

Smithson S. (1994). Information retrieval evaluation in practice: a case study approach. Information Processing \& Management, Vol. 30, No. 2, pp. 205-221. (88)

Smoliar S. and Zhang H.J. (1994). Content-based video indexing and retrieval. IEEE Multimedia, Vol. 1, No. 2, pp. 62-72. (17)

Span G. (1994). LITES: an intelligent tutoring system shell for legal education. International Yearbook of Law Computers and Technology (eds. K. Russel and R. Jones), pp. 103-113. Carfax Publishing Company. (73)

Sparck Jones K. (1995). Reflections on TREC. Information Processing \& Management, Vol. 31, No. 3, pp. 291-314. (91)

St. Pierre M., Fullton J., Gamiel K., Goldman J., Kahle B., Kunze J., Moris H., and Schiettecatte F. (1993). WAIS server, WAIS workstation, WAIS forwarder for UNIX, release 1.1. Technical report, Wais Inc. $(21,26,27,28,29,31,32,35)$

Su L.T. (1992). Evaluation measures for interactive information retrieval. Information Processing \& Management, Vol. 28, No. 4, pp. 503-516.

Su L.T. (1994). The relevance of recall and precision in user evaluation. Journal of the American Society for Information Science, Vol. 45, No. 3, pp. 207-217. (90)

Sudarshan B. (1979). Development of reference retrieval system with simultaneous building of thesaurus. Lib. Sc., Vol. 16, No. 3, pp. 77-83. (30)

Tague-Sutcliffe J.M. (1981). The pragmatics of information retrieval experimentation. Information Retrieval Experiment (ed. K. Sparck Jones), Chapter 5, pp. 59-102. Butterworths, London, England. (89)

Tague-Sutcliffe J.M. (1992). The pragmatics of information retrieval experimentation, revisited. Information Processing \& Management, Vol. 28, No. 4, pp. $467-490 . \quad(88,91,93)$

Turtle H. and Croft W.B. (1991). Evaluation of an inference network-based retrieval model. ACM Transactions on Information Systems, Vol. 9, No. 3, pp. 187-222. (28) 
Turtle H. (1993). Text retrieval in the legal world. Tutorial notes presented at the Fourth International Conference on AI and Law. $(18,22,35)$

Turtle H. (1994). Natural language vs. Boolean query evaluation: A comparison of retrieval performance. Proceedings of the Seventeenth Annual International ACM SIGIR Conference on Research and Development in Information Retrieval (eds. W.B. Croft and C.J. van Rijsbergen), pp. 212-220, Springer-Verlag, London, England. (20, 29)

Utting K. and Yankelovich N. (1989). Context and orientation in hypermedia networks. ACM Transactions on Information Systems, Vol. 7, No. 1, pp. 58-84. (50)

Van den Herik H.J. (1997). Kennistechnologie en normware. Informatie, Vol. 39, No. 6, pp. 8-15. (3)

Van der Pol R.W. (1996). A device for query composition. Technical Report CS 9602, Department of Computer Science, Maastricht University. $(13,64)$

Van Rijsbergen C.J. (1979). Information Retrieval. Butterworths, London, England, 2nd edition. (90)

Voorhees E.M. (1993). Using WordNet to disambiguate word senses for text retrieval. Proceedings of the Sixteenth Annual International ACM SIGIR Conference on Research and Development in Information Retrieval, Linguistic Analysis, pp. 171-180. (27)

Wall L. and Schwartz R.L. (1991). Programming perl. O'Reilly \& Associates, Sebastopol, CA. (69)

Waterworth J. and Chignell M. (1991). A model for information exploration. Hypermedia, Vol. 3, No. 1, pp. 35-58. (9, 96)

Webster (1975). Webster's New Collegiate Dictionary. G.z.C. Mirriam Co.

Wells G.A. (1972). Goethe, Johann Wolfgang von. Dictionary of Scientific Biography (ed. C.C. Gillispie), Vol. 5, pp. 442-446. Charles Scribner's Sons, New York, NY.

Wiesman F. and Panhuysen R.G.A.M. (1995). Computer assisted diagnosis in paleopathology. Journal of Paleopathology, Vol. 7, No. 2, p. 149.

Wiesman F. and Hasman A. (1997). Graphical information retrieval by browsing meta-information. Computer Methods and Programs in Biomedicine, Vol. 53, No. 3, pp. 135-152. (65) 
Wiesman F., Hasman A., and Van den Herik H.J. (1997). Information retrieval: An overview of system characteristics. International Journal of Medical Informatics, Vol. 47, No. 1-2, pp. 5-26. $(15,16)$

Wilbur W.J. (1992). An information measure of retrieval performance. Information Systems, Vol. 17, No. 4, pp. 283-298.

Yoon Y., Choi K., Kim G., and Shin D. (1992). A hybrid knowledge-based approach to information retrieval. Microprocessing and Microprogramming, Vol. 35, No. 1, pp. 329-336. $(18,27,28,35)$ 



\section{Index}

AltaVista, 37-39

ANOVA, 103, 105

ANS

-CS, 51-54, 64, 170, 174

-FB, 51-54, 58-62, 64, 170, 174

$-\mathrm{M}, 51-53,59$

-MS, 51-53, 57, 64, 170, 174

-S, 51-53, 58-63, 170, 174

-SD , 51, 52, 62, 170, 174

search behaviour in, 119-121

session, 52

site, 52-54, 62

Archimedes project, 1, 10-12, 46, 53, 74,140

backtrack facility, $81,82,120$

black-box experiment, 88

bookmark, 81, 84, 125

browsing behaviour, 7-10

$\mathrm{C}++, 70,75$

chance hit, 116, 129

classification, $37,69,72,138$

clustering, 35-36, 144

cognitive load, 78,138

combination of key terms, $75,83-85$, $111,120-122,124,125,129$, $135,140,143,144$

communication, $4-5,7,11,52,65,73$

conceptual distance, 79,80

conceptual queries, 24, 30-31, 37, 95

Cook's distance, 105, 111

DAP, $42,43,45,47,48,51,60,62,63$, $65,66,68,70,72-74,77,139$
DEMO/FM, 53

Dexter model, 44, 45

diagnostic experiment, 88

disorientation, $12,50,59,62,63,75$, $78,81,113-115,133$

document

copy, 48,53

definition of, $42-43$

descriptor, 48

document set, 22, 31, 87-89, 91, 93, $95,128,134-137,139,140$, 142,143

effectiveness, 3, 4, 16, 20, 22, 27-29, $34,35,49,50,63,87-90,93$, $94,96,108,109,115,116$, $128,134-139$

efficiency, 50, 87, 90, 93, 94, 96, 115, $128,134,135,137,139$

Embase, 144

EP-X, 29

Excite, 37-39

expert system, $26,62,69,73,138,144$

experts' agreement, 101-103

fact type, 47, 54-56, 59-61

field searching, 26, 31-32, 37, 38, 122

filter mechanism, 50, 60, 63, 81-82, $125,132,139,144$

formal language, $4,5,29,46,47,59$, 131

formula medium kind, 45-48, 73, 131 full text, $3,25,26,31,33,37,38,95$, $122,129,139$ 
fuzzy queries, $25,26,32$

genetic algorithm, 34

graph drawing, 78

graphical query language, $16,24,27$, $29-30,50$

GraphLog, 30

Grateful Med, 95

Harvest, 36

Hawthorne effect, 93, 98

history list, $63,81,144$

homogeneity problem, 138

HotBot, 38

HTML, 70, 74

hypermedia, 12, 36, 76, 77, 133

hypertext, $8,12,22,26,32-33,36,44-$ $45,50,69,71,72,77,81,133$, 138

indexing, 4, 6-7, 12, 16, 24, 31, 39, $70-72,95,135,144$

information need, 4-5, 7, 9-11, 15, 16, $29,59,64,88,89,91-93,137$, 140,143

Infoseek, 38

inter-document link, 44, 45, 62

Internet, $7,36,39,51$

intra-document link, 44, 45, 62

IR model, 15, 17, 24, 29, 37, 50, 132, 140

Java content handlers, 62,74

knowledge, 3-8

document, 65

domain, 5-7, 11, 13, 24, 26-28, $36,49,50,62,65,69,71,94$, $96,105,128,137,139,140$, 144

procedural, 5, 11, 138

strategic, 6, 11

Knowledge Finder, 95, 136 laboratory experiment, $13,88,134$

LiveTopics, 37

LSD test, 105

LyberWorld, 50

Lycos, 37-39

machine learning, 23, 24, 26, 33-34, 37

Mann-Whitney, 109

Medline, 2, 3, 7, 63, 64, 70, 71, 76, 95, $111,123,124,127,134-136$, $140,142-144,147$

Medline Information Network, 69-71, $82,135,142,143$

mental model, 5

$\mathrm{MeSH}, 28,31,70,71,95,99,135,140$, 141

meta-information, 8, 11-12, 49-51, 65, $131,132,138-140,143$

meta-Web-browser, 77, 144

metabrowsing, 50, 76, 132, 140

metaschema, 47, 51, 53, 56-58, 132

MIME, 74

MOMSpider, 36

multimedia, 10-12, 17, 36, 59, 71, 82

natural language, $4,16,24,25,27,29$, $32,38,47,55,136$

object class, 56,57

operational experiment, 88,134

Paleopathology Information Network, $\mathrm{x}, 72$

PaperChase, 95

pearl growing, 6,7

performance, $12,29,33,39,91,93,94$, $134,136,139$

Perl, 69

$\mathrm{PL}+, 73$

precision

definition of, 16,89

presentation form, 43, 46-49 
problem-based learning, 3, 147

question-answering system, 16

ranked output, $18,21,26,34-35,37$, $38,89,92,99,143$

recall

definition of, 16, 89

relevance

definition of, 92-93

relevance feedback, $24,27-28,38$

relevant set, 93, 134

reliability, 4,7

representation type, 45-46

result set, 92

retrieved set, $87,88,92,99,138$

RGB, 67

Rijsbergen's measure

definition of, 90

scientific information, 1-3, 137

search engine, 36-39

search intermediary, 24, 26-27, 29, 37,

64,139

semantic network, 11, 28, 36, 53, 65, $68,69,71$

separator, $66-68$

Silverplatter, 18, 70, 95, 136

spring embedder algorithm, 59, 78

stopword, 16, 18

storage representation, 45-47, 49

subheading, $71,82,84,122,126,142$

subject directory, 36-39

tag, $66,73,74$

test collection, 91

thinking-aloud protocol, 90

Thoth-II, 50

Topic, 17

topology, 78, 140, 144

Tower Model, 45

Transnational Income Tax Information Network, 73, 144
TREC, 91

UMLS, $28,59,63,69-71,95,113,114$, $129,135,136,139-142,144$

user model, 33

Virtual Hospital Information Network, 59, 71-72, 77

Web robot, 36, 37, 39

WordNet, 27, 144

Yahoo, 38-39 



\section{Summary}

Storage and retrieval of information are the most important applications for computer systems. In particular, retrieving information is considered a challenging task. It is usually performed by an information retrieval system (IR system). In the domain of science, IR is essential for producers of information, for users of information, and for students. However, finding the right information with the current IR systems is difficult. A perfect IR system would retrieve precisely the relevant documents; no more, no fewer. Several studies have shown that the effectiveness of existing IR systems is rather inadequate. There is a variety of causes, of which five prominent ones are addressed in this research.

Whereas the traditional approach in IR is to request the users to describe the information they are looking for (by formulating a query), the novel approach is to let them browse through the available information. In our research, we propose to browse through a graphical representation of meta-information. We call this way of browsing metabrowsing. The meta-information is contained in a structure dubbed the Information Network, which combines four kinds of meta-information: information about (1) the domain discussed in the documents, (2) the contents of the documents, (3) the relations between the documents, and (4) the locations of the documents. The system in which the Information Network is implemented is called the ARChimedes Network System (ANS).

Our approach addresses five problems of IR: (1) the problem of human-computer communication (by performing browsing-oriented search instead of query-oriented search), (2) the problem of human-human communication (by using a conceptoriented system instead of a string-oriented one), (3) the problem of missing or faulty procedural knowledge (by using an intuitive user interface), (4) the problem of missing or faulty strategic knowledge of search request formulation (by using an intuitive user interface), and (5) the problem of missing or faulty domain knowledge (by giving the domain representation a central position in the system).

Our problem statement reads as follows:

Is it possible to provide facilities for searching various information items by allowing the user to browse in a graphical way through metainformation, making it an improvement over existing methods? 
Chapter 2 proposes a set of thirteen characteristics for the comparison of queryoriented IR systems known from the literature. We used this set to decide which characteristics ANS should possess. After weighing pros and cons, the following characteristics were agreed upon: domain knowledge, graphical query language, conceptual queries, field searching, and hypertext integration.

Chapter 3 describes the various kinds of information and the notion of a document. A document is stored information that is declared to be a document by its producer. A special kind of document is defined, called formula, for expressions in formal languages. Two particular kinds of formula documents are databases and knowledge bases.

The chapter continues with a discussion of the ANS architecture. It is based on an extension of relational databases providing meta-information about the documents. The architecture consists of two parts: the ANS server (ANS-S) and a database, the Information Network. ANS-S consists of a browser mechanism, a query mechanism, and server data (ANS-SD). The browser and query mechanisms are used to access the Information Network. The contents of the documents to be retrieved are presented by external programs managed by ANs. In the design, the Information Network is a relational database management system (RDBMS) with the following elements: a fact base (ANS-FB), a conceptual schema (ANS-CS), and a metaschema (ANS-MS). These are accessed by ANS-S through the ANS manager.

Instead of an RDBMS a customised database is used in the first ANS prototype. It implements ANS-FB and ANS-SD; ANS-CS and ANS-MS are not explicitly represented. The prototype was informally evaluated by four test persons. A number of shortcomings is identified; the next chapter describes how they are alleviated.

Chapter 4 presents a detailed description of the ANS implementation. The syntax of the Information Network is defined. The central elements of an Information Network are nodes and links: a node represents either a concept or a document; a link represents a relation between two nodes. The relations can be chosen at will. With these two elements it is possible to represent the meta-information identified in Chapter 1. To illustrate the possibilities of the Information Network four instances are presented: the Medline Information Network (aimed at plain IR), the Virtual Hospital Information Network (aimed at browsing through hypertext), the Paleopathology Information Network (aimed at classification), and the Transnational Income Tax Information Network (aimed at the integration of expert systems and IR).

Browsing in a graphical way through the meta-information requires visualization of the meta-information. The nodes and links are represented on the screen as boxes and lines. Since an Information Network is generally too large to be displayed on a computer screen, an algorithm was devised for displaying a part of the graph. The user selects which part of the graph is displayed. Several navigation aids were added to prevent the user from becoming lost in the Information Network. 
Chapter 5 provides a general overview of how to evaluate an IR system (based on the literature) and describes the experimental design used for the evaluation of ANS.

The literature shows that for some issues in experimental design the optimal choice is obvious, whereas for other issues the theory is vague, many times leading to arbitrary decisions. Well-known measures for the performance of an IR system are efficiency and effectiveness. The former is a relatively clear measure. The latter is associated with the poorly understood concept of relevance. The most widely used measures for effectiveness are recall (the part of the relevant documents that is actually retrieved) and precision (the part of the retrieved documents that is actually relevant). Rijsbergen's measure combines recall and precision in a balanced way.

The objective of the ANS evaluation experiment is to study the difference in performance of a system like ANS and a standard Boolean IR system. We define performance as a combination of effectiveness, efficiency, and user satisfaction. A number of test persons was asked questions about the medical domain. The questions were of the form "Find documents about..."; the test persons had to answer them using two IR systems. Since we expected ANS to be beneficial especially for users with little domain knowledge test persons were divided into two groups: (1) test persons with relatively little domain knowledge, and (2) test persons with relatively much domain knowledge. The test persons were recruited under medical students. Second-year students were assigned to the first group, fourth-year students were assigned to the second group. Domain experts formulated questions to be answered by all test persons. Each test person answered one question with ANs, and two with WinSpirs (the Boolean system), or vice versa. The result of a question for a test person was a set of documents. For each question the union of the results was determined. For each union two domain experts judged the relevance of each document. From these relevance judgements recall, precision, and Rijsbergen's measure could be determined for the two systems. The efficiency of a system was measured by the average search time of the users. To establish the user satisfaction, for each question the test persons were asked how satisfied they were with the result. Finally, the test persons were interviewed about their experiences with the systems.

Chapter 6 presents the results of the ANS evaluation. The main conclusions are:

- In the overall comparison, ANS and WinSpirs are equally effective. However, for one individual question ANS is more effective, and also for the second-year students one may argue that ANS is more effective.

- ANS is less efficient than WinSpirs for fourth-year students, and ANS is more efficient than WinSpirs for second-year students.

- In the overall comparison, the test persons are equally satisfied with ANS and WinSpirs. However, the fourth-year students are more satisfied with WinSpirs than with ANS. 
- The advantage of ANS over WinSpirs in terms of effectiveness, efficiency, and user satisfaction is greater for second-year students than for fourth-year students.

Chapter 7 summarizes and discusses the conclusions of the previous chapters, addresses the hypotheses and problem statement formulated in Chapter 1, and proposes directions for future research.

The first part of the problem statement, stating that metabrowsing is feasible, is answered by an experimental proof: it is feasible and this has been demonstrated. The second part, stating that metabrowsing is an improvement over existing methods, is answered with a careful 'no, however'. The experiment did neither show a significantly better performance for ANS as compared to WinSpirs, nor the reverse. A number of possible explanations for not finding a difference has been listed. The innovation of ANS is that it represents documents and domain in one representation in a relatively unconstrained way, and that the representation can be browsed both in its entirety and in separate layers (by applying filters).

Future research should be directed at improving the ANS browser, the domain representation in general, and particularly the domain representation for medical Information Networks. Finally, ANs has a wide applicability by its further integration into other information systems. 


\section{Samenvatting}

Opslag en ontsluiting van informatie zijn de belangrijkste toepassingen van computer systemen. Vooral ontsluiting van informatie wordt beschouwd als een uitdagende opgave. Dit wordt meestal verricht door een information retrieval systeem (IR-systeem). Binnen het wetenschapsdomein is IR essentieel voor producenten van informatie, gebruikers van informatie en studenten. Met de huidige IR-systemen is het echter moeilijk de juiste informatie te vinden. Een perfect IR-systeem zou precies de relevante documenten vinden: niet meer, niet minder. Verscheidene onderzoeken hebben aangetoond dat de effectiviteit van de bestaande IR-systemen te wensen overlaat. Hiervoor is een aantal oorzaken aan te noemen; dit onderzoek richt zich op vijf belangrijke oorzaken.

Daar waar de traditionele IR-benadering de gebruiker zijn informatiebehoefte laat beschrijven (middels een zoekvraag), laat de hier gekozen benadering de gebruiker grasduinen (browsing) door de beschikbare informatie. In ons onderzoek stellen we voor te grasduinen door meta-informatie die we grafisch weergeven. We noemen deze wijze van grasduinen metabrowsing. De meta-informatie is gevat in een structuur met de naam Informatienetwerk. Deze structuur combineert vier soorten metainformatie, te weten: informatie over (1) het domein waar de documenten betrekking op hebben, (2) de inhoud van de documenten, (3) de relaties tussen de documenten en (4) de locaties van de documenten. Het systeem waarin het Informatienetwerk is geïmplementeerd heet het ARCHIMEDEs Netwerk Systeem (ANS).

Onze benadering richt zich op vijf problemen binnen IR: (1) het probleem van de mens-computer communicatie (door de gebruiker te laten zoeken door grasduinen in plaats van vragen), (2) het probleem van de mens-mens communicatie (door een concept-georiënteerd systeem te gebruiken in plaats van een string-georiënteerd systeem), (3) het probleem van onvolledige of verkeerde procedurele kennis (door een intuitieve gebruikers-interface te bieden), (4) het probleem van onvolledige of verkeerde strategische kennis voor het formuleren van zoekvragen (door een intuïtieve gebruikers-interface te bieden) en (5) het probleem van onvolledige of verkeerde domeinkennis (door de domeinrepresentatie een centrale positie in het systeem te geven). 
Onze probleemstelling luidt als volgt:

Is het mogelijk voorzieningen te bieden voor het zoeken naar verschillende soorten informatie door de gebruiker op grafische wijze te laten grasduinen in meta-informatie, en wel zodanig, dat het een verbetering is ten opzichte van de bestaande methoden?

Hoofdstuk 2 geeft een verzameling van dertien karakteristieken voor het vergelijken van zoekvraag-georiënteerde IR-systemen die besproken worden in de literatuur. We gebruiken deze verzameling om te bepalen welke karakteristieken ANS zou moeten hebben. Na voor- en nadelen te hebben afgewogen, werd gekozen voor de volgende karakteristieken: domeinkennis, grafische zoekvraagtaal, conceptuele zoekvragen, zoeken in velden, en hypertext-integratie.

Hoofdstuk 3 beschrijft de verschillende soorten informatie en het begrip 'document'. Een document is opgeslagen informatie die tot document is bestempeld door zijn producent. A speciaal soort document wordt geïntroduceerd, genaamd formula, voor uitdrukkingen in formele talen. Twee bijzondere soorten formula-documenten zijn databases (gegevensbanken) en knowledge bases (kennisbanken).

Het hoofdstuk bespreekt vervolgens de ANS-architectuur. Deze is gebaseerd op een uitbreiding van relationele databases die meta-informatie over documenten biedt. De architectuur bestaat uit twee delen: de ANS-server (ANS-S) en een database, het Informatienetwerk. ANS-S bestaat uit een grasduinmechanisme, een zoekvraagmechanisme en server-gegevens (server data, ANS-SD). De grasduin- en zoekvraagmechanismen geven de gebruiker toegang tot het Informatienetwerk. De inhoud van de gezochte documenten wordt getoond door externe programma's die door ANS worden aangestuurd. In het ontwerp is het Informatienetwerk een relationeel database management systeem (RDBMS) met de volgende drie elementen: een feitenbank (ANS-FB), een conceptueel schema (ANS-CS) en een metaschema (ANS-MS). Ze worden aangesproken door ANS-S via de ANS-manager.

In het eerste prototype wordt een speciaal gemaakte database gebruikt in plaats van een RDBMS. Het verwezenlijkt de ANS-FB en ANS-SD; ANS-CS en ANS-MS zijn niet expliciet gerepresenteerd. Het prototype is informeel geëvalueerd door vier proefpersonen. Er is een aantal tekortkomingen geconstateerd; het volgende hoofdstuk beschrijft hoe ze zijn aangepakt.

Hoofdstuk 4 beschrijft de implementatie van ANS in detail. De syntaxis van het Informatienetwerk wordt vastgelegd. De hoofdelementen van een Informatienetwerk zijn knopen en verbindingen: een knoop stelt of een concept of een document voor; een verbinding stelt een relatie tussen twee knopen voor. De relaties kunnen willekeurig gekozen worden. Met deze twee elementen is het mogelijk de meta-informatie te representeren die in hoofdstuk 1 wordt genoemd. Ter illustratie van de mogelijkheden 
van het Informatienetwerk worden vier voorbeelden gegeven: het Medline Informatienetwerk (gericht op gewone IR), het Virtual Hospital Informatienetwerk (gericht op grasduinen door hypertext), het Paleopathologie Informatienetwerk (gericht op classificatie) en het Grensoverschrijdende Inkomstenbelasting Informatienetwerk (gericht op de integratie van expertsystemen en IR).

Op grafische wijze grasduinen door meta-informatie vereist visualisatie van de meta-informatie. De knopen en verbindingen worden op een scherm weergegeven door rechthoeken en lijnen. Aangezien een Informatienetwerk doorgaans te groot is om op een computerscherm te worden getoond is een algoritme ontwikkeld dat een deel van het netwerk laat zien. De gebruiker selecteert welk deel moet worden getoond. Een aantal navigatiehulpmiddelen is toegevoegd om te voorkomen dat de gebruiker verdwaalt in het Informatienetwerk.

Hoofdstuk 5 bespreekt (op basis van de literatuur) hoe een IR-systeem in het algemeen kan worden geëvalueerd en beschrijft het onderzoeksontwerp voor de evaluatie van ANS.

De literatuur laat zien dat voor sommige evaluatiekwesties de juiste keuze duidelijk is. Voor andere kwesties is de theorie echter vaag, hetgeen vaak leidt tot ongegronde besluiten. Bekende maten voor de prestaties van een IR-systeem zijn efficiëntie en effectiviteit. De eerste is een relatief duidelijke maat, de tweede is afhankelijk van het onduidelijke begrip 'relevantie'. De meest gebruikte maten voor effectiviteit zijn recall (het deel van de relevante documenten dat is gevonden) en precision (het deel van de gevonden documenten dat relevant is). Rijsbergens maat is een afgewogen combinatie van recall en precision.

Doel van het ANS evaluatie-experiment is het verschil in prestaties te onderzoeken tussen een systeem als ANS en een standaard Booleaans IR-systeem. We definiëren prestatie als een combinatie van effectiviteit, efficiëntie en tevredenheid van de gebruiker. Een aantal proefpersonen kreeg vragen voorgelegd over het medisch domein. De vragen waren van de vorm "Zoek documenten over..."; de proefpersonen moesten ze beantwoorden met gebruik van twee IR-systemen. Aangezien we verwachtten dat ANS vooral nuttig zou zijn voor gebruikers met weinig domeinkennis werden de proefpersonen verdeeld over twee groepen: (1) proefpersonen met relatief weinig domeinkennis en (2) proefpersonen met relatief veel domeinkennis. De proefpersonen werden geworven onder studenten geneeskunde. Tweedejaars studenten werden in de eerste groep geplaatst, vierdejaars studenten in de tweede. Domeinexperts formuleerden vragen die door alle proefpersonen moesten worden beantwoord. Elke proefpersoon beantwoordde één vraag met ANS en twee met WinSpirs (het Booleaanse IR-systeem) of andersom. Het resultaat voor een proefpersoon was een verzameling documenten. Voor elke vraag werd de vereniging van de resultaten vastgesteld. Voor elke vereniging beoordeelden twee domeinexperts de relevantie van elk document. Met deze relevantiebepalingen konden voor de beide systemen de recall, de precision en Rijsbergens maat worden vastgesteld. De efficiëntie van een systeem werd gemeten door de gemiddelde zoektijd van de gebruikers. De tevredenheid van de ge- 
bruikers werd bepaald door voor elke vraag de proefpersonen te vragen hoe tevreden ze waren met het behaalde resultaat.

Hoofdstuk 6 toont de resultaten van de ANS evaluatie. De belangrijkste conclusies zijn:

- In de totale vergelijking zijn ANS en WinSpirs even effectief. Voor één vraag echter is ANS effectiever. Ook voor de tweedejaars studenten kan worden gesteld dat ANS effectiever is.

- ANS is minder efficiënt dan WinSpirs voor vierdejaars studenten en ANS is efficiënter dan WinSpirs voor tweedejaars studenten.

- In de totale vergelijking zijn de proefpersonen even tevreden over ANS als over WinSpirs. Echter, de vierdejaars studenten zijn meer tevreden over WinSpirs dan over ANS.

- Het voordeel dat ANS heeft op WinSpirs in termen van effectiviteit, efficiëntie en tevredenheid van de gebruiker is groter voor tweedejaars studenten dan voor vierdejaars studenten.

Hoofdstuk 7 resumeert en bespreekt de conclusies uit de voorgaande hoofdstukken. De hypothesen en de probleemstelling worden besproken en richtingen voor toekomstig onderzoek worden voorgesteld.

Het eerste deel van de probleemstelling, dat stelt dat metabrowsing mogelijk is, wordt positief beantwoord met een empirisch bewijs. Het tweede deel, dat stelt dat metabrowsing een verbetering is over bestaande methoden, wordt beantwoord met een voorzichtig 'nee, echter'. In het experiment zijn de prestaties van ANS noch significant beter, noch significant slechter dan die van WinSpirs. Er wordt een aantal mogelijke verklaringen gegeven voor het niet vinden van een verschil.

De vooruitgang die ANS biedt is gelegen in de representatie van documenten en domein die relatief gezien vrij is en in de mogelijkheid de representatie zowel in zijn geheel als in aparte lagen (door filters toe te passen) te begrasduinen.

Toekomstig onderzoek zou zich moeten richten op verbetering van het ANS grasduinmechanisme en de domeinrepresentatie in het algemeen en die voor medische Informatienetwerken in het bijzonder. Tenslotte wordt vastgesteld dat ANs breed toepasbaar is en derhalve geintegreerd dient te worden in andere informatiesystemen. 


\section{Curriculum Vitae}

Floris Wiesman werd geboren op 12 april 1967 te Nijmegen. De middelbareschoolopleiding werd gevolgd aan het College Hageveld te Heemstede. In 1986 behaalde hij het Atheneum diploma. Hierna volgde hij de studie Informatica aan de Universiteit van Amsterdam. In 1992 studeerde hij af in de richting Kunstmatige Intelligentie en Robotiek. In hetzelfde jaar kreeg hij een aanstelling als assistent in opleiding bij de vakgroep Informatica aan de Universiteit Maastricht, en werd hij gedetacheerd bij de vakgroep Medische Informatica. Sinds oktober 1997 is hij wetenschappelijk programmeur bij de vakgroep Informatica. 